\title{
Consistency of Proof-of-Stake Blockchains with Concurrent Honest Slot Leaders
}

\author{
Aggelos Kiayias ${ }^{1,3}$, Saad Quader ${ }^{2}$, and Alexander Russell ${ }^{2,3}$ \\ ${ }^{1}$ University of Edinburgh $\quad{ }^{2}$ University of Connecticut $\quad{ }^{3} \mathrm{IOHK}$
}

July 30,2020

\begin{abstract}
We improve the fundamental security threshold of eventual consensus Proof-of-Stake (PoS) blockchain protocols under longest-chain rule, reflecting for the first time the positive effect of rounds with concurrent honest leaders. Current analyses of these protocols reduce consistency to the dynamics of an abstract, round-based block creation process that is determined by three probabilities:

- $p_{\mathrm{A}}$, the probability that a round has at least one adversarial leader;

- $p_{\mathrm{h}}$, the probability that a round has a single honest leader; and

- $p_{\mathrm{H}}$, the probability that a round has multiple, but honest, leaders.

We present a consistency analysis that achieves the optimal threshold $p_{\mathrm{h}}+p_{\mathrm{H}}>p_{\mathrm{A}}$. This is a first in the literature and can be applied to both the simple synchronous setting and the setting with bounded delays. Moreover, we achieve the optimal consistency error $e^{-\Theta(k)}$ where $k$ is the confirmation time. We also provide an efficient algorithm to explicitly calculate these error probabilities in the synchronous setting.

All existing consistency analyses either incur a penalty for rounds with concurrent honest leaders, or treat them neutrally. Specifically, the consistency analyses in Ouroboros Praos (Eurocrypt 2018) and Genesis (CCS 2018) assume that the probability of a uniquely honest round exceeds that of the other two events combined (i.e., $p_{\mathrm{h}}-p_{\mathrm{H}}>p_{\mathrm{A}}$ ); the analyses in Sleepy Consensus (Asiacrypt 2017) and Snow White (Fin. Crypto 2019) assume that a uniquely honest round is more likely than an adversarial round (i.e., $p_{\mathrm{h}}>p_{\mathrm{A}}$ ). In addition, previous analyses completely break down when uniquely honest rounds become less frequent, i.e., $p_{\mathrm{h}}<p_{\mathrm{A}}$. These thresholds determine the critical trade-off between the honest majority, network delays, and consistency error.

Our new results can be directly applied to improve the consistency guarantees of the existing protocols. We complement these results with a consistency analysis in the setting where uniquely honest slots are rare, even letting $p_{\mathrm{h}}=0$, under the added assumption that honest players adopt a consistent chain selection rule.
\end{abstract}

\section{Introduction}

Proof-of-Stake (PoS) blockchain protocols have emerged as a viable alternative to resource-intensive Proof-of-Work (PoW) blockchain protocols such as Bitcoin and Ethereum. These PoS protocols are organized in rounds (which we call slots in this paper); their most critical algorithmic component is a leader election procedure which determinesfor each slot-a subset of participants with the authority to add a block to the blockchain. Existing security analyses of these protocols are logically divided into two components: the first reasons about the properties of the leader election process, the second reasons about the combinatorial properties of the blockchains that can be produced by an idealized leader schedule in the face of adaptive adversarial control of some participants. An attractive side effect of this structure is that the combinatorial considerations can be treated independently of other aspects of the protocol. A recent article of Blum et al. [3] gave an axiomatic treatment of this combinatorial portion of the analysis which we extend in this paper.

These common combinatorial arguments can be formulated with very little information about the leader election process. Specifically, current analyses focus on three parameters: 
- $p_{\mathrm{h}}$, the probability that a slot is uniquely honest, having a single honest leader;

- $p_{\mathrm{H}}$, the probability that a slot is multiply honest, having multiple, but honest, leaders; and

- $p_{\mathrm{A}}$, the probability that a slot has at least one adversarial leader.

Our major contribution is a generic, rigorous guarantee of consistency under the most desirable assumption $11 p_{\mathrm{h}}+$ $p_{\mathrm{H}}>p_{\mathrm{A}}$ that achieves optimal consistency error $\exp (-\Theta(k))$ as a function of confirmation time $k$. Our analysis can be directly applied to existing protocols to improve their consistency guarantees.

To contrast this with existing literature, the analysis of Ouroboros Praos [5] and Ouroboros Genesis [1] require the threshold assumption $p_{\mathrm{h}}-p_{\mathrm{H}}>p_{\mathrm{A}}$ to achieve the optimal consistency error of $e^{-\Theta(k)}$. Note how multiply honest slots actually detract from security, appearing negatively in the basic security threshold. The consistency analyses in Snow White [2] and Sleepy Consensus [10] assume an improved threshold $p_{\mathrm{h}}>p_{\mathrm{A}}$; however, they only establish a consistency error bound of $e^{-\Theta(\sqrt{k})}$. Note here that multiply honest slots appear neutrally. All existing analyses break down if $p_{\mathrm{h}}<p_{\mathrm{A}}$, i.e., when the uniquely honest slots are less probable than the adversarial slots.

Multiply honest slots may arise by design, e.g., when each player checks privately whether he is a leader. They may also occur naturally in the non-synchronous setting when the time between the broadcast of two blocks is exceeded by network delay-in this case the party issuing the later block may not be aware of the earlier block which can result the two blocks sharing the same chain history, a de facto incidence of multiple honest leaders. The role of these slots is rather delicate: while it is good for the system to have many honest blocks, concurrent blocks can help the adversary in creating two long, diverging blockchains that might jeopardize the consistency property. Our new analysis shows that this second effect can be mitigated, achieving consistency error bound of $e^{-\Theta(k)}$ under the (tight) assumption $p_{\mathrm{h}}+p_{\mathrm{H}}>p_{\mathrm{A}}$.

Our results and contributions. As described above, we show for the first time that PoS blockchain protocols using the longest-chain rule can achieve a consistency error of $e^{-\Theta(k)}$ under the desirable condition $p_{\mathrm{h}}+p_{\mathrm{H}}>p_{\mathrm{A}}$. This improves the security guarantee of all "longest chain rule" PoS protocols such as Praos [5], Genesis [1], and Snow White [2] (we remark that other PoS protocols such as Algorand [9] operate in a different setting where explicit participation bounds are assumed and forks can be prevented). We discuss our results in more detail before turning to the model and proofs.

Our analysis in the simple synchronous model achieves the same asymptotic error bound as in [4] -the tightest result in the literature-under a much weaker assumption, namely $p_{\mathrm{h}}+p_{\mathrm{H}}>p_{\mathrm{A}}$. Thus PoS protocols can in fact achieve consistency with $p_{\mathrm{h}}<p_{\mathrm{A}}$, a regime beyond reach of all previous analyses. When uniquely honest slots are rare (i.e., when $p_{\mathrm{h}}$ is very small), our bound has the desired dependence on $p_{\mathrm{h}}$. Moreover, when $p_{\mathrm{H}}=0$ (i.e., all honest slots are in fact uniquely honest), we exactly recover the bound in [4]. We also give an algorithm to explicitly compute the probability that a given slot encounters a consistency violation under the idealized leader election mechanism. The time and space required by this algorithm is cubic in the length of the protocol execution.

Next, we consider a variant model where the honest players use a consistent tie-breaking rule when selecting the longest chain. (I.e., when a fixed set of blockchains of equal length are presented to a collection of honest players, they all select the same chain. In previous models, the adversary had the right to break such ties by influencing network delivery.) Assuming $p_{\mathrm{h}}+p_{\mathrm{H}}>p_{\mathrm{A}}$, we prove that the consistency error bound in this model is identical to the $e^{-\Theta(k)}$ bound in [4] even when $p_{\mathrm{h}}=0$. No existing analysis survives in this regime.

$\Delta$-synchronous setting. In the $\Delta$-synchronous communication setting, all messages are delivered with at most a $\Delta$ delay. Our results mentioned above can be transferred to this setting using the $\Delta$-synchronous to synchronous reduction approach used in the Ouroboros Praos analysis [5]. Thus, we can achieve a consistency error probability of $e^{-\Theta(k)}$ in this setting as well. This analysis is presented in Section 8

A technical overview. We initially work in the synchronous communication model and extend the synchronous combinatorial framework of [3] to accommodate multiply honest slots.

\footnotetext{
${ }^{1}$ Consistency is unachievable in the case $p_{\mathrm{h}}+p_{\mathrm{H}}<p_{\mathrm{A}}$. See [7] for a detailed discussion of the honest majority assumption.
} 
First, our analysis focuses on a combinatorial event called a "Catalan slot.2 2 Catalan slots are honest slots $c$ with the property that any interval containing $c$ possesses strictly more honest slots-with any number of honest leaders - than adversarial ones. The analysis of [2] and [10] introduced this basic concept, though they counted only uniquely honest slots. In comparison with their analysis, then, our treatment has two important advantages: first of all, we let multiply honest slots count in the analysis and, additionally, we achieve strikingly stronger error bounds: specifically, we achieve optimal settlement error of $\exp (-\theta(k))$ rather than $\exp (-\theta(\sqrt{k}))$.

A Catalan slot $c$ acts as a barrier for the adversary in that if an honest blockchain from a slot $h<c$ is padded with adversarial blocks and presented to an honest observer at slot $c+1$, the observer will never adopt this blockchain. As a result, the chains adopted by this honest observer must contain some block from slot $c$. Note that this is true even if $c$ is multiply honest. A critical observation is that a slot is Catalan if and only if all competitive blockchains in future slots contain at least one block from this slot. Thus, if a Catalan slot $c$ is uniquely honest, all blockchains that are eligible to be adopted by future honest players must contain the (only) honest block issued from slot $c$. We call this the "Unique Vertex Property" (UVP). Note how the UVP is reminiscent of the "Common Prefix Property" (CP) in the literature. Thus, together, the UVP and Catalan slots act as a conduit between consistency violations and the underlying stochastic process.

Our major technical challenge is to bound the probability that Catalan slots are infrequent. Here we break away entirely from the analysis of [2] and approach the question using the theory of generating functions and stochastic dominance. We find an exact generating function for a related event and use this, by dominance, to control the undesirable event that a long window of slots is devoid of Catalan slots. This yields asymptotically optimal settlement bounds.

Finally, it follows from the discussion above that if two consecutive slots are Catalan then any subsequent honest block must contain, in its prefix, a block from each of these slots. In a setting where all honest players use a consistent longest-chain selection rule, we show that both slots have UVP as well. Since Catalan slots can be multiply honest, PoS protocols can achieve a consistency error bound of $e^{-\Theta(k)}$ in this model even if $p_{\mathrm{h}}=0$.

In a separate line of reasoning, in Section 6 we generalize the fork-theoretic framework of Blum et al. [3] for the multi-leader setting. Here, we characterize the UVP in terms of the so-called "relative margin," a combinatorial property of a given slot. We describe an adversary who optimally attacks the UVP of all slots, simultaneously. Next, we prove a recurrence relation for relative margin. Suppose each slot is independently and identically chosen (by the leader election mechanism) to be either uniquely honest, multiply honest, or adversarial. The recurrence relation mentioned above then leads to an algorithm to explicitly compute the probability that a given slot encounters a consistency violation; see Section 6.6 In contrast, the Catalan slot-centric characterization of the UVP gives us only an asymptotic bound on this probability. It can be concluded that the fork-framework, after all, is expressive enough to capture consistency violations in the multi-leader setting.

Outline. We specify our model in Section 2 and focus on a specific consistency property called " $k$-settlement." This section also contains our main theorems; the proofs are deferred to Section 4 In Section 3 we describe amplifications to the fork framework of [3] in order to explore the relationship between Catalan slots and the UVP. In Section 4 we present two bounds on the stochastic events of interest, e.g., the rarity of a Catalan slot; these bounds lead to short proofs of the main theorems. The proofs of these bounds are presented next in Section 5 which contains all of our probabilistic arguments.

Section 6 contains an alternative treatment of the UVP via fork-theoretic notions of [3]. Along the way, it describes an optimal adversary who simultaneously attacks the consistency of all slots. It also describes an algorithm to compute explicit values for the probability of consistency violations. The proofs of two important theorems from this section are presented subsequently in Section 7

Our treatment of the $\Delta$-synchronous setting is presented in Section 8 In Section 9 we treat the traditional Common Prefix (CP) violations using our bounds on the UVP.

In Appendix $\AA$ we characterize common prefix violations in the presence of multiply honest slots using "balanced forks" from [3] (and, importantly, without using Catalan slots).

\footnotetext{
${ }^{2}$ The name is a nod to the Catalan number in combinatorics: The $n$th Catalan number $C_{n}$ is the number of strings $w \in\{0,1\}^{2 n}$ so that every prefix $x$ of $w$ satisfies $\#_{0}(x) \geq \#_{1}(x)$.
} 


\section{The model and our main theorems}

We study the behavior of the elementary longest-chain rule algorithm, carried out by a collection of participants:

- In each round, each participant collects all valid blockchains from the network; if a participant is a leader in the round, he adds a block to the longest chain and broadcasts the result.

Here, "valid" indicates that any block appearing in the chain was indeed issued by a leader from the associated slot; in the PoS setting, this property is guaranteed with digital signatures.

We begin by studying this algorithm in the simple, synchronous model posited by Blum et. al [3]. The model adopts a synchronous communication network in the presence of a rushing adversary: in particular,

A0. Any message broadcast by an honest participant at the beginning of a particular slot is received by the adversary first, who may decide strategically and individually for each recipient in the network whether to inject additional messages and in which order all messages are to be delivered prior to the conclusion of the slot.

See the comments prior to Section 2.1 for further discussion of this network assumption. A variant of this adversarial message-ordering is presented in Section 2.3 The $\Delta$-synchronous communication model is handled in Section 8

Given this, it is easy to describe the behavior of the longest-chain rule when carried out by a group of honest participants with the extra guarantee that exactly one is elected as leader in a slot: Assuming that the system is initialized with a common "genesis block" corresponding to $s l_{0}$, the players observe a common, linearly growing blockchain:

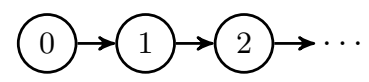

Here node $i$ represents the block broadcast by the leader of slot $i$ and the arrows represent the direction of increasing time.

The blockchain axioms: Informal discussion. The introduction of adversarial participants or multiple slot leaders complicates the family of possible blockchains that could emerge from this process. To explore this in the context of our protocols, we work with an abstract notion of a blockchain which ignores all internal structure. We consider a fixed assignment of leaders to time slots, and assume that the blockchain uses a proof mechanism to ensure that any block labeled with slot $s l_{t}$ was indeed produced by a leader of slot $s l_{t}$; this is guaranteed in practice by appropriate use of a secure digital signature scheme.

Specifically, we treat a blockchain as a sequence of abstract blocks, each labeled with a slot number, so that:

A1. The blockchain begins with a fixed "genesis" block, assigned to slot $s l_{0}$.

A2. The (slot) labels of the blocks are in strictly increasing order.

It is further convenient to introduce the structure of a directed graph on our presentation, where each block is treated as a vertex; in light of the first two axioms above, a blockchain is a path beginning with a special "genesis" vertex, labeled 0 , followed by vertices with strictly increasing labels that indicate which slot is associated with the block.

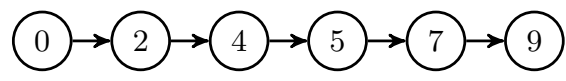

The protocols of interest call for honest players to add a single block during any slot. In particular:

A3. Let $k \geq 1$ be an integer. If a slot $s l_{t}$ was assigned to $k$ honest players but no adversarial players, then $k$ blocks are created-during the entire protocol-each having the label $s l_{t}$.

Recall that blockchains are immutable in the sense that any block in the chain commits to the entire previous history of the chain; this is achieved in practice by including with each block a collision-free hash of the previous block. These properties imply that any chain that includes a block issued by an honest player must also include that block's associated prefix in its entirety.

As we analyze the dynamics of blockchain algorithms, it is convenient to maintain an entire family of blockchains at once. As a matter of bookkeeping, when two blockchains agree on a common prefix, we can glue together the associated paths to indicate this, as shown below. 


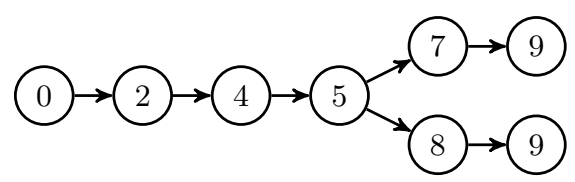

When we glue together many chains to form such a diagram, we call it a "fork"-the precise definition appears below. Observe that while these two blockchains agree through the vertex (block) labeled 5, they contain (distinct) vertices labeled 9; this reflects two distinct blocks associated with slot 9 which, in light of the axiom above, may be produced by either an adversarial participant assigned to slot 9 or two honest participants, both assigned to slot 9 .

Finally, as we assume that messages from honest players are delivered before the next slot begins, we note a direct consequence of the longest chain rule:

A4. If two honestly generated blocks $B_{1}$ and $B_{2}$ are labeled with slots $s l_{1}$ and $s l_{2}$ for which $s l_{1}<s l_{2}$, then the length of the unique blockchain terminating at $B_{1}$ is strictly less than the length of the unique blockchain terminating at $B_{2}$.

Recall that the honest participant(s) assigned to slot $s l_{2}$ will be aware of the blockchain terminating at $B_{1}$ that was broadcast by an honest player in slot $s l_{1}$ as a result of synchronicity; according to the longest-chain rule, $B_{2}$ must have been placed on a chain that was at least this long. In contrast, not all participants are necessarily aware of all blocks generated by dishonest players, and indeed dishonest players may often want to delay the delivery of an adversarial block to a participant or show one block to some participants and show a completely different block to others.

Characteristic strings, forks, and the formal axioms. Note that with the axioms we have discussed above, whether or not a particular fork diagram (such as the one just above) corresponds to a valid execution of the protocol depends on how the slots have been awarded to the parties by the leader election mechanism. We introduce the notion of a "characteristic" string as a convenient means of representing information about slot leaders in a given execution.

Definition 1 (Characteristic string). Let $s l_{1}, \ldots, s l_{n}$ be a sequence of slots. A characteristic string $w$ is an element of $\{\mathrm{h}, \mathrm{H}, \mathrm{A}\}^{n}$. The string $w$ is consistent with a particular execution of a blockchain protocol on these slots if for each $t \in[n]$, (i) if $w_{t}=\mathrm{A}$, the slot $s l_{t}$ is assigned to at least one adversarial participant, (ii) if $w_{t}=\mathrm{h}$, the slot sl is assigned to a unique, honest participant, and (iii) if $w_{t}=\mathrm{H}$, the slot sl $l_{t}$ is assigned to at least one honest participant and no adversarial participants.

Observe that when an execution corresponds to a characteristic string $w$, it also corresponds to any string obtained from $w$ by replacing $\mathrm{h}$ symbols with $\mathrm{H}$ symbols.

For two strings $x$ and $w$ on the same alphabet, we write $x \prec w$ if and only if $x$ is a strict prefix of $w$. Similarly, we write $x \preceq w$ if and only if either $x=w$ or $x \prec w$. The empty string $\varepsilon$ is a prefix to any string. If $w_{t} \in\{\mathrm{h}, \mathrm{H}\}$, we say that "sl$l_{t}$ is honest" and otherwise, we say that "sl is adversarial." With this discussion behind us, we set down the formal object we use to reflect the various blockchains adopted by honest players during the execution of a blockchain protocol. This definition formalizes the blockchains axioms discussed above.

Definition 2 (Fork). Let $w \in\{\mathrm{h}, \mathrm{H}, \mathrm{A}\}^{n}, P=\left\{i: w_{i}=\mathrm{h}\right\}$, and $Q=\left\{j: w_{j}=\mathrm{H}\right\}$. A fork for the string $w$ consists of a directed and rooted tree $F=(V, E)$ with a labeling $\ell: V \rightarrow\{0,1, \ldots, n\}$. We insist that each edge of $F$ is directed away from the root vertex and further require that

(F1) the root vertex $r$ has label $\ell(r)=0$;

(F2) the labels of vertices along any directed path are strictly increasing;

(F3) each index $i \in P$ is the label of exactly one vertex of $F$ and each index $j \in Q$ is the label of at least one vertex of $F$; and

(F4) for any indices $i, j \in P \cup Q$, if $i<j$ then the depth of a vertex with label $i$ is strictly less than the depth of a vertex with label $j$. 


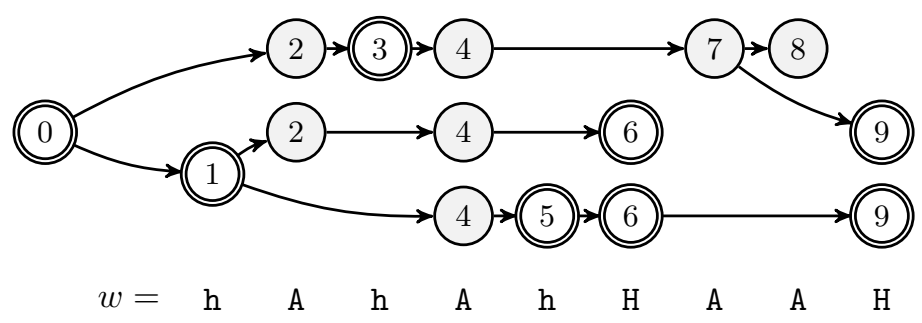

Figure 1: A fork $F$ for the characteristic string $w=$ hAhAhHAAH; vertices appear with their labels and honest vertices are highlighted with double borders. Note that the depths of the (honest) vertices associated with the honest indices of $w$ are strictly increasing. Note, also, that this fork has three disjoint paths of maximum depth. In addition, two honest vertices have label 6 and two more have label 9, indicating the fact that two honest leaders are associated with each of the (honest) slots 6 and 9. Honest vertices with the same label are concurrent and, therefore, cannot extend each other. Note that the two honest vertices with label 6 extend different vertices with the same depth. This is allowed since any tie in the longest-chain rule is broken by the adversary.

If $F$ is a fork for the characteristic string $w$, we write $F \vdash w$. The conditions (F1) $(\mathrm{F} 4)$ are analogues of the axioms A1 A4 above. The formal reflection of axiom A3 by condition (F3) deserves further comment: We have chosen a definition of characteristic string that does not indicate the number of honest victories in cases where there may be many; in particular, the symbol H may be associated with any positive number of (honest) vertices in the fork. Indeed, we even permit a fork to have a single honest vertex associated with such a symbol, which enlarges the class of forks under consideration for a particular characteristic string. This strengthens our results by effectively giving the adversary the option to treat $\mathrm{H}$ symbols as $\mathrm{h}$ symbols. See Fig. 1 for an example fork.

A final notational convention: If $F \vdash x$ and $\hat{F} \vdash w$, we say that $F$ is a prefix of $\hat{F}$, written $F \sqsubseteq \hat{F}$, if $x \preceq w$ and $F$ appears as a consistently-labeled subgraph of $\hat{F}$. (Specifically, each path of $F$ appears, with identical labels, in $\hat{F}$.)

Let $w$ be a characteristic string. The directed paths in the fork $F \vdash w$ originating from the root are called tines; these are abstract representations of blockchains. (Note that a tine may not terminate at a leaf of the fork.) We naturally extend the label function $\ell$ for tines: i.e., $\ell(t) \triangleq \ell(v)$ where the tine $t$ terminates at vertex $v$. The length of a tine $t$ is denoted by length $(t)$.

Viable tines. The longest-chain rule dictates that honest players build on chains that are at least as long as all previously broadcast honest chains. It is convenient to distinguish such tines in the analysis: specifically, a tine $t$ of $F$ is called viable if its length is no smaller than the depth of any honest vertex $v$ for which $\ell(v) \leq \ell(t)$. A tine $t$ is viable at slot $s$ if the length of the portion of $t$ appearing over slots $0, \ldots, s$ is no smaller than the depths of any honest vertices labeled from these slots. (As noted, the properties (F3) and (F4) together imply that an honest observer at slot $s$ will only adopt a viable tine.) The honest depth function $\mathbf{d}: P \cup Q \rightarrow[n]$, defined as $\mathbf{d}(i)=$ $\max _{t \in F}\{$ length $(t): \ell(t)=i\}$, gives the largest depth of the (honest) vertices associated with an honest slot; by (F4), $\mathbf{d}(\cdot)$ is strictly increasing.

\subsection{Slot settlement and the Unique Vertex Property}

We are now ready to explore the power of an adversary in this setting who has corrupted a (perhaps evolving) coalition of the players. We focus on the possibility that such an adversary can violate the consistency of the honest players' blockchains. In particular, we consider the possibility that, at some time $t$, the adversary conspires to produce two maximum-length blockchains that diverge prior to a previous slot $s \leq t$; in this case honest players adopting the longest-chain rule may clearly disagree about the history of the blockchain after slot $s$. We call such a circumstance a settlement violation.

To express this in our abstract language, let $F \vdash w$ be a fork corresponding to an execution with characteristic string $w$. Such a settlement violation induces two viable tines $t_{1}, t_{2}$ with the same length that diverge prior to a particular slot of interest. We record this below. 
The $(\mathcal{D}, T ; s, k)$-settlement game

1. A characteristic string $w \in\{\mathrm{h}, \mathrm{H}, \mathrm{A}\}^{T}$ is drawn from $\mathcal{D}$. (This reflects the results of the leader election mechanism.)

2. Let $A_{0} \vdash \varepsilon$ denote the initial fork for the empty string $\varepsilon$ consisting of a single node corresponding to the genesis block.

3. For each slot $s l_{t}, t=1, \ldots, T$ in increasing order:

(a) (Honest slot.) This case pertains to $w_{t} \in\{\mathrm{h}, \mathrm{H}\}$. If $w_{t}=\mathrm{h}$ then $\mathcal{A}$ sets $k=1$. If $w_{t}=\mathrm{H}$ then $\mathcal{A}$ chooses an arbitrary integer $k \geq 1$. The challenger is then given $k$ and the fork $A_{t-1} \vdash w_{1} \ldots w_{t-1}$. He must determine a new fork $F_{t} \vdash w_{1} \ldots w_{t}$ by adding $k$ new vertices (all labeled with $t$ ) to $A_{t-1}$. Each new vertex is added at the end of a maximum-length path in $A_{t-1}$. If there are multiple candidates for this path, $\mathcal{A}$ may break the tie. If $k \geq 2$, multiple vertices (all with label $t$ ) may be added at the end of the same path.

(b) (Adversarial slot.) If $w_{t}=\mathrm{A}$, this is an adversarial slot. $\mathcal{A}$ may set $F_{t} \vdash w_{1} \ldots w_{t}$ to be an arbitrary fork for which $A_{t-1} \sqsubseteq F_{t}$.

(c) (Adversarial augmentation.) $\mathcal{A}$ determines an arbitrary fork $A_{t} \vdash w_{1} \ldots, w_{t}$ for which $F_{t} \sqsubseteq A_{t}$.

Recall that $F \sqsubseteq F^{\prime}$ indicates that $F^{\prime}$ contains, as a consistently-labeled subgraph, the fork $F$.

$\mathcal{A}$ wins the settlement game if slot $s$ is not $k$-settled in some fork $A_{t}, t \geq s+k$.

${ }^{a}$ It is possible that all maximum-length tines are honest. In the settlement game considered in [4], at least one of these tines was adversarial.

Definition 3 (Settlement with parameters $s, k \in \mathbb{N}$ ). Let $n \in \mathbb{N}$ and let $w$ be a characteristic string of length $n$. Let $t \in[s+k, n]$ be an integer, $\hat{w} \preceq w,|\hat{w}|=t$, and let $F$ be any fork for $\hat{w}$. We say that a slot $s$ is not $k$-settled in $F$ if $F$ contains two maximum-length tines $\mathcal{C}_{1}, \mathcal{C}_{2}$ that "diverge prior to $s$," i.e., they either contain different vertices labeled with $s$, or one contains a vertex labeled with $s$ while the other does not. Otherwise, we say that slot $s$ is $k$-settled in $F$. We say that slot $s$ is $k$-settled in $w$ if, for each $t \geq s+k$, it is $k$-settled in every fork $F \vdash \hat{w}$ where $\hat{w} \preceq w,|\hat{w}|=t$.

Definition 4 (Bottleneck Property (BP) and Unique Vertex Property (UVP)). Let $w \in\{\mathrm{h}, \mathrm{H}, \mathrm{A}\}^{T}$ be a characteristic string. A slot $s \in[T]$ is said to have the bottleneck property in $w$ if, for any fork $F \vdash w$ and any $k \geq s+1$, every tine viable at the onset of slot $k$ contains, as its prefix, some vertex with label s. Slot $s$ is said to have the Unique Vertex Property if, for any fork $F \vdash w$, there is a unique vertex $u \in F$ with label s so that for any $k \geq s+1$, all tines viable at the onset of slot $k$ contain, as their common prefix, the vertex $u$.

Thus if a uniquely honest slot in $w$ has the bottleneck property, it has the UVP as well. As a consistency property, UVP has several advantages over slot settlement. First, it easily implies the slot settlement property: let $w \in\{\mathrm{h}, \mathrm{H}, \mathrm{A}\}^{T}, s \in[T]$, and $k \in[T-s]$.

$$
\text { If a slot } t \in[s, s+k] \text { has UVP in } w \text { then } s \text { is } k \text {-settled in } w \text {. }
$$

In addition, UVP has a straightforward characterization using "Catalan slots" (see Theorem 3 ) and "relative margin" (see Lemma 1); these characterizations are amenable to stochastic analysis. Finally, since UVP is structurally reminiscent of the traditional common prefix (CP) violations, UVP easily implies CP. The analogous statement "settlement implies CP," however, requires a lengthy proof both in [3] and in our framework. See Appendix A for details.

\subsection{Adversarial attacks on settlement time; the settlement game}

To clarify the relationship between forks and the chains at play in a canonical blockchain protocol, we define a gamebased model below that explicitly describes the relationship between forks and executions. By design, the probability 
that the adversary wins this game is at most the probability that a slot $s$ is not $k$-settled.

Consider the ( $\mathcal{D}, T ; s, k)$-settlement game (presented in the box), played between an adversary $\mathcal{A}$ and a challenger $\mathcal{C}$ with a leader election mechanism modeled by an ideal distribution $\mathcal{D}$. Intuitively, the game should reflect the ability of the adversary to achieve a settlement violation; that is, to present two maximum-length viable blockchains to a future honest observer, thus forcing them to choose between two alternate histories which disagree on slot $s$. The challenger plays the role(s) of the honest players during the protocol.

It is important to note that the game bestows the player $\mathcal{A}$ with the power to choose the number of honest vertices in a multiply honest slot. Note that this setting makes the player strictly more powerful and, importantly, implies that the game is completely determined by the choices made by $\mathcal{A}$ (i.e., the actions of the challenger are deterministic). Consequently, in Definition 5 we can use a single, implicit universal quantifier over all strategies $\mathcal{A}$; no choices of the challenger are actually necessary to fully describe the game.

Definition 5 (Settlement insecurity). Let $\mathcal{D}$ be a distribution on $\{\mathrm{h}, \mathrm{H}, \mathrm{A}\}^{T}$. Let $w \sim \mathcal{D}$ be the string used in the first step of a $(\mathcal{D}, T ; s, k)$-settlement game $G$. The $(s, k)$-settlement insecurity of $\mathcal{D}$ is defined as

$$
\mathbf{S}^{s, k}[\mathcal{D}] \triangleq \max _{\substack{\hat{\hat{w}} \preceq w \\
|\hat{w}| \geq s+k}} \max _{F \vdash \hat{w}} \operatorname{Pr}\left[\begin{array}{c}
F \text { has two maximum-length tines } \\
\text { that diverge prior to slot } s
\end{array}\right] .
$$

Note that the probability in the right-hand side is the same as the probability that $\mathcal{A}$ wins $G$.

Note that in typical PoS settings the distribution $\mathcal{D}$ is determined by the combined stake held by the adversarial players, the leader election mechanism, and the dynamics of the protocol. The most common case (as seen in Snow White [2], Ouroboros [8], and Ouroboros Praos [5]) guarantees that the characteristic string $w=w_{1} \ldots w_{T}$ is drawn from an i.i.d. distribution for which $\operatorname{Pr}\left[w_{i}=\mathrm{A}\right] \leq(1-\epsilon) / 2$ for some $\epsilon \in(0,1)$; here the constant $(1-\epsilon) / 2$ is directly related to the stake held by the adversary. Some settings involving adaptive adversaries (e.g., Ouroboros Praos [5]) yield a weaker martingale-type guarantee that $\operatorname{Pr}\left[w_{i}=\mathrm{A} \mid w_{1}, \ldots, w_{i-1}\right] \leq(1-\epsilon) / 2$. We can easily handle both types of distributions in our analysis since the former distribution "stochastically dominates" the latter. As a rule, we denote the probability distribution associated with a random variable using uppercase script letters.

Definition 6 (Stochastic dominance). Let $X$ and $Y$ be random variables taking values in some set $\Omega$ endowed with a partial order $\leq$. We say that $X$ stochastically dominates $Y$, written $Y \preceq X$, if $\mathcal{X}(A) \geq \mathcal{Y}(A)$ for all monotone sets $A \subseteq \Omega$, where a set $A \subseteq \Omega$ is called monotone if $a \in A$ implies $a^{\prime} \in A$ for all $a \leq a^{\prime}$. As a special case, when $\Omega=\mathbb{R}$, $Y \preceq X$ if $\operatorname{Pr}[X \geq \Lambda] \geq \operatorname{Pr}[Y \geq \Lambda]$ for every $\Lambda \in \mathbb{R}$. We extend this notion to probability distributions in the natural way.

Throughout the paper, we adopt the following partial order on $\{\mathrm{h}, \mathrm{H}, \mathrm{A}\}^{T}$ : If $T=1$, define $\mathrm{h}<\mathrm{H}<\mathrm{A}$. Otherwise, for two strings $x a, y b \in\{\mathrm{h}, \mathrm{H}, \mathrm{A}\}^{T},|a|=|b|=1, x a \leq y b$ if and only if $x \leq y$ and $a \leq b$. When $x \leq y$, one might say that $y$ is "more adversarial" than $x$ : indeed, if $F \vdash x$ and $x \leq y$ then $F \vdash y$ so that any settlement violation for $x$ induces a settlement violation for $y$.

Definition $7\left(\left(\epsilon, p_{\mathrm{h}}\right)\right.$-Bernoulli condition). Let $T \in \mathbb{N}, \epsilon \in(0,1)$, and $p_{h} \in[0,(1+\epsilon) / 2]$. Define $p_{\mathrm{A}}=(1-\epsilon) / 2$ and $p_{\mathrm{H}}=1-p_{\mathrm{A}}-p_{\mathrm{h}}$. A random variable $w=w_{1} \ldots w_{T}$ taking values in $\{\mathrm{h}, \mathrm{H}, \mathrm{A}\}^{T}$ is said to satisfy the $\left(\epsilon, p_{\mathrm{h}}\right)$-Bernoulli condition if each $w_{i}, i \in[T]$, is independent and identically distributed as follows: $\operatorname{Pr}\left[w_{i}=\sigma\right]=p_{\sigma}$ for $\sigma \in\{\mathrm{h}, \mathrm{H}, \mathrm{A}\}$. The distribution of $w$ is also said to satisfy the $\left(\epsilon, p_{\mathrm{h}}\right)$-Bernoulli condition.

We frequently use the notation $p_{\mathrm{H}}$ and $p_{\mathrm{A}}$ in the context of such a random variable when $\epsilon$ and $p_{\mathrm{h}}$ can be inferred from context.

Theorem 1 (Main theorem). Let $\epsilon, p_{\mathrm{h}} \in(0,1)$ and $s, k, T \in \mathbb{N}$. Let $\mathcal{B}$ be a distribution on length-T characteristic strings satisfying the $\left(\epsilon, p_{\mathrm{h}}\right)$-Bernoulli condition. Then $\mathbf{S}^{s, k}[\mathcal{B}] \leq \exp \left(-k \cdot \Omega\left(\min \left(\epsilon^{3}, \epsilon^{2} p_{\mathrm{h}}\right)\right)\right.$. Furthermore, let $\mathcal{W}$ be a distribution on $\{\mathrm{h}, \mathrm{H}, \mathrm{A}\}^{T}$ so that $\mathcal{W} \preceq \mathcal{B}$. Then $\mathbf{S}^{s, k}[\mathcal{W}] \leq \mathbf{S}^{s, k}[\mathcal{B}]$. (Here, the asymptotic notation hides constants that do not depend on $\epsilon$ or $k$.)

Note that the quantity $p_{\mathrm{h}}$ above cannot be zero. We present the proof in Section 4 In Section 6 we give a characterization of the UVP which allows us to explicitly compute $\mathbf{S}^{s, k}[\mathcal{B}]$; see Theorem 5 and Section 6.6 
Analysis in the $\Delta$-synchronous setting. The security game above most naturally models a blockchain protocol over a synchronous network with immediate delivery (because each "honest" play of the challenger always builds on a fork that contains the fork generated by previous honest plays). However, the model can be easily adapted to protocols in the $\Delta$-synchronous model by applying the $\Delta$-reduction mapping of [5] (which is specifically designed to lift the synchronous analysis to the $\Delta$-synchronous setting). These details appear in Section 8

Public leader schedules. One attractive feature of this model is that it gives the adversary full information about the future schedule of leaders. The analysis of some protocols indeed demand this (e.g., Ouroboros, Snow White). Other protocols-especially those designed to offer security against adaptive adversaries (Praos, Genesis)-in fact contrive to keep the leader schedule private. Of course, as our analysis is in the more difficult "full information" model, it applies to all of these systems.

Bootstrapping multi-phase algorithms; stake shift. We remark that several existing proof-of-stake blockchain protocols proceed in phases, each of which is obligated to generate the randomness (for leader election, say) for the next phase based on the current stake distribution. The blockchain security properties of each phase are then individually analyzed-assuming clean randomness-which yields a recursive security argument; in this context the game outlined above precisely reflects the single phase analysis.

\subsection{A consistent longest-chain selection rule}

Let us modify axiom $\mathbf{A 0}$ as follows:

$\mathbf{A 0}^{\prime}$. In addition to axiom A0 an arbitrary but consistent longest-chain tie-breaking rule is used by all honest participants.

As a consequence, if two honest participants observe the same set of blockchains of maximum length, they will extend the same blockchain.

Definition 8 (Bivalent characteristic string). Let $s l_{1}, \ldots, s l_{n}$ be a sequence of slots. A bivalent characteristic string $w$ is an element of $\{\mathrm{H}, \mathrm{A}\}^{n}$ defined for a particular execution of a blockchain protocol on these slots so that for $t \in[n]$, $w_{t}=\mathrm{A}$ if $\mathrm{sl}_{t}$ is assigned to an adversarial participant, and $w_{t}=\mathrm{H}$ otherwise.

The definition of a fork for a bivalent characteristic string is identical to Definition 2 (somewhat simplified as a bivalent string does not contain any $\mathrm{h} \mathrm{symbol})$. Also note that the $(\epsilon, 0)$-condition from Definition 7 is well-defined for bivalent characteristic strings.

Let $w$ be a bivalent characteristic string, $F$ a fork for $w$, and $F^{\prime}$ a fork for $w \mathrm{H}$ so that $F \sqsubseteq F^{\prime}$ and any honest vertex in $F^{\prime} \backslash F$ has label $|w|+1$. If $F$ contains a maximum-length adversarial tine, there is no guarantee that two honest observers at slot $|w|+1$ will agree on the longest chain: the adversary may chose to expose the adversarial chain to one and not the other. In this case, we say that $F$ has a tie for the longest-chain rule-or, in short, that $F$ has an LCR tie. When there is no LCR tie (that is, no maximum-length adversarial tine), all honest slot leaders at slot $|w|+1$ necessarily extend the same honest tine determined by the consistent longest-chain tie-breaking rule.

Theorem 2 (Main theorem; consistent tie-breaking). Let $\epsilon \in(0,1)$ and $s, k, T \in \mathbb{N}$. Let $\mathcal{B}$ be a distribution on length-T bivalent characteristic strings satisfying the $(\epsilon, 0)$-Bernoulli condition. Let $\mathcal{W}$ be a distribution on $\{\mathrm{H}, \mathrm{A}\}^{T}$ so that $\mathcal{W} \preceq \mathcal{B}$. Then $\mathbf{S}^{s, k}[\mathcal{W}] \leq \mathbf{S}^{s, k}[\mathcal{B}] \leq \exp \left(-k \cdot \Omega\left(\epsilon^{3}(1+O(\epsilon))\right)\right.$ ). (Here, the asymptotic notation hides constants that do not depend on $\epsilon$ or $k$.)

The proof is deferred to Section 4 Note that the theorem above states that a PoS protocol can achieve optimal consistency error even with a leader election scheme that produces no uniquely honest slots. In contrast, Theorem 1 requires a non-zero probability for uniquely honest slots. 


\section{Unique Vertex Property via Catalan slots}

As we have outlined before, if slot $t$ in a characteristic string $w$ has the Unique Vertex Property (UVP) then the slots $s=1, \ldots, t$ are settled in every fork for $w$. The goal of this section is to characterize when a slot has the UVP. (In Section 6 we show an alternative way to characterize the UVP; see Lemma 1)

We start with laying down some structural properties of forks. Next, we define the so-called Catalan slots and show that if a slot is Catalan then in every fork, all sufficiently long blockchains must contain a block from that slot. Next, we show that this implication is actually an equivalence. Finally, we revisit the above implication assuming that the honest players use a consistent longest-chain tie-breaking rule.

\subsection{Viable blockchains}

A vertex of a fork is said to be honest if it is labeled with an index $i$ such that $w_{i} \in\{\mathrm{h}, \mathrm{H}\}$; otherwise, it is said to be adversarial.

Definition 9 (Tines, length, and height). Let $F \vdash w$ be a fork for a characteristic string. A tine of $F$ is a directed path starting from the root. For any tine $t$ we define its length to be the number of edges in the path, and for any vertex $v$ we define its depth to be the length of the unique tine that ends at $v$. If a tine $t_{1}$ is a strict prefix of another tine $t_{2}$, we write $t_{1} \prec t_{2}$. Similarly, if $t_{1}$ is a non-strict prefix of $t_{2}$, we write $t_{1} \preceq t_{2}$. The longest common prefix of two tines $t_{1}, t_{2}$ is denoted by $t_{1} \cap t_{2}$. That is, $\ell\left(t_{1} \cap t_{2}\right)=\max \left\{\ell(u): u \preceq t_{1}\right.$ and $\left.u \preceq t_{2}\right\}$. The height of a fork (as is usual for a tree) is the length of the longest tine, denoted by height $(F)$.

Let $F \vdash x y$ and two tines $t_{1}, t_{2} \in F$ are disjoint over $y$. We say that these tines are $y$-disjoint; equivalently, we also say that $t_{1}$ is $y$-disjoint with $t_{2}$.

When an adversary builds a fork, it is natural to imagine that he "grows" an existing fork by adding new vertices and edges.

Definition 10 (Fork prefixes). Let $w, x \in\{\mathrm{h}, \mathrm{H}, \mathrm{A}\}^{*}$ so that $x \preceq w$. Let $F, F^{\prime}$ be two forks for $x$ and $w$, respectively. We say that $F$ is a prefix of $F^{\prime}$ if $F$ is a consistently labeled subgraph of $F^{\prime}$. That is, all vertices and edges of $F$ also appear in $F^{\prime}$ and the label of any vertex appearing in both $F$ and $F^{\prime}$ is identical. We denote this relationship by $F \sqsubseteq F^{\prime}$.

When speaking about a tine that appears in both $F$ and $F^{\prime}$, we place the fork in the subscript of relevant properties.

For any string $x$ (on any alphabet) and a symbol $\sigma$ in that alphabet, define $\#_{\sigma}(x)$ as the number of appearances of $\sigma$ in $x$. When a characteristic string $w \in\{\mathrm{h}, \mathrm{H}, \mathrm{A}\}^{T}$ is fixed from the context, we extend this notation to subintervals of $[T]$ in a natural way: For integers $i, j \in[T], i \leq j$, let $I=[i, j] \subset[T]$ be a closed interval and define $\#_{\sigma}(I)=\#_{\sigma}\left(w_{i} \ldots w_{j}\right)$ for $\sigma \in\{\mathrm{h}, \mathrm{H}, \mathrm{A}\}$. A characteristic string $w$ is called $\mathrm{hH}$-heavy if $\#_{\mathrm{h}}(w)+\#_{\mathrm{H}}(w)>\#_{1}(x)$; otherwise, it is called A-heavy. For a given characteristic string $w$ of length $T$, an interval $I=[i, j] \subseteq[T]$ is called A-heavy if the substring $w_{i} \ldots w_{j}$ is A-heavy.

Adversarial extensions. Let $x, y$ be two characteristic strings and $|y| \geq 0$. Let $F$ be a fork for $x$ and let $B$ be an honest tine in $F$. We say that $B$ has an adversarial extension if there is a fork $F^{\prime} \vdash x y, F \sqsubseteq F^{\prime}$ and an adversarial tine $t \in F^{\prime}$ so that $B \prec t$ and $B$ is the last honest vertex on $t$. Note that $t$ can be made disjoint with any $F$-tine over the interval $[\ell(B)+1, \ell(t)]$.

Viable adversarial extensions and $\mathrm{A}$-heaviness. Let $w \in\{\mathrm{h}, \mathrm{H}, \mathrm{A}\}^{T}, s \in[T+1]$, and $F \vdash w_{1} \ldots w_{s-1}$ an arbitrary fork. Let $B \in F$ be an honest vertex and $t$ a maximum-length honest tine in $F$. Consider the following statements:

(a) $B$ has an adversarial extension viable at the onset of slot $s$.

(b) The interval $I=[\ell(B)+1, s-1]$ is A-heavy.

(c) length $(t)=\#_{\mathrm{h}}(I)+\#_{\mathrm{H}}(I)+\operatorname{length}(B)$.

Fact 1. $(a) \Longrightarrow(b)$ In addition, if we assume (c) then $(b) \Longrightarrow(a)$ 
Proof.

(a) implies (b) Let $F^{\prime} \vdash w_{1} \ldots w_{s-1}$ be a fork so that $F \sqsubseteq F^{\prime}$ and $B$ has an adversarial extension $t^{\prime} \in F^{\prime}$ viable at the onset of slot $s$. Considering the interval $I$, the longest honest tine in $F^{\prime}$ grows by at least $\#_{\mathrm{h}}(I)+\#_{\mathrm{H}}(I)$ vertices. Since the viable tine $t^{\prime}$ contains only adversarial vertices from the interval $I$, it follows that $\#_{\mathrm{A}}(I)$ must be at least $\#_{\mathrm{h}}(I)+\#_{\mathrm{H}}(I)$. Hence, $I$ is A-heavy.

(c) and (b) implies (a) Since $I$ is A-heavy, $I$ contains at least $\#_{\mathrm{h}}(I)+\#_{\mathrm{H}}(I)=$ length $(t)-\operatorname{length}(B)$ adversarial slots. Thus, we can augment $B$ by adding length $(t)-\operatorname{length}(B)$ adversarial vertices from these slots so that the resulting adversarial extension is viable at the onset of slot $s$.

Corollary 1. Let $w$ be a characteristic string, $F$ be any fork for $w$, and let $t$ be any tine in $F$. Let $B_{1}$ and $B_{2}$ be two honest vertices on $t$ such that $(i) \ell\left(B_{1}\right)<\ell\left(B_{2}\right)$, (ii) $t$ contains only adversarial vertices from $I=\left[\ell\left(B_{1}\right)+1, \ell\left(B_{2}\right)-1\right]$, and (iii) $t$ contains at least one vertex from $I$. Then $I$ is A-heavy.

Proof. By assumption, the honest vertex $B_{2}$ builds on some adversarial tine $t^{\prime}$ that is viable at the onset of slot $\ell\left(B_{2}\right)$ and, importantly, contains $B_{1}$ as its last honest vertex. By Fact 1 the interval $I$ is A-heavy.

\subsection{Catalan slots and the UVP}

Below, we define the so-called Catalan slots and show, in Theorems 3 and 4 that certain Catalan slots have the UVP.

Definition 11 (Catalan slot). Let $w \in\{\mathrm{h}, \mathrm{H}, \mathrm{A}\}^{T}$ be a characteristic string and let $s \in[T]$ be an integer. $s$ is called a left-Catalan slot in $w$ if, for any integer $\ell \in[s]$, the interval $[\ell, s]$ is hH-heavy in $w$. $s$ is called a right-Catalan slot in $w$ if, for any integer $r \in[s, T]$, the interval $[s, r]$ is $\mathrm{hH}$-heavy in $w$. Finally, $s$ is called a Catalan slot in $w$ if it is both left- and right-Catalan in $w$.

Observe that a left- or right-Catalan slot must be honest. In addition, the slot before a left-Catalan (resp., after a right-Catalan) slot must be honest as well. Thus the slots adjacent to a Catalan slot must be honest. A Catalan slot $c$ acts as a barrier for adversarial tine extensions in that in any fork, every tine viable at the onset of slot $c+1$ must be honest.

Fact 2. Let $w \in\{\mathrm{h}, \mathrm{H}, \mathrm{A}\}^{T}$ be a characteristic string and s a left-Catalan slot in $w$. In any fork for $w$, every viable tine at the onset of slot $s+1$ is an honest tine from slot $s$.

Proof. Let $\tau$ be the longest tine with label $s$. ( $\tau$ is an honest tine. If $s$ is a uniquely honest slot, $\tau$ is unique. Otherwise, $\tau$ is unique up to tie-breaking among equally-long tines.) We claim that all adversarial tines $t \in F, \ell(t) \leq s-1$ are strictly shorter than $\tau$. Suppose, towards a contradiction, that $t$ is a viable adversarial tine at the onset of slot $s+1$, i.e., $\ell(t) \leq s-1$ and length $(t) \geq \operatorname{length}(\tau)$. Let $B$ be the last honest vertex on $t$; necessarily, $\ell(B)<s$. According to Fact 1 the interval $[\ell(B)+1, s]$ is A-heavy. But this contradicts the assumption that $s$ is a left-Catalan slot. Hence the adversarial tine $t$ cannot be viable.

Observation 1. If $s$ is a Catalan slot for $w$, Fact 2implies that in every fork for $w$, an honest slot leader at slot $s+1$ always builds on top of an honest tine with label $s$; this tine, in fact, will have the maximum length among all tines with label $s$.

Fact 3. Let $w \in\{\mathrm{h}, \mathrm{H}, \mathrm{A}\}^{T}$ be a characteristic string. If an honest slot in $w$ has the bottleneck property then it is a Catalan slot.

Proof. Let $s \in[T]$ be an honest slot in $w$. We will prove the contrapositive: namely, that if $s$ is not Catalan then $s$ does not have the bottleneck property.

Suppose $s$ is not a Catalan slot. Then there must be some $a, b \in[T]$ so that $I=[a, b]$ is the largest A-heavy interval which includes $s$. Necessarily, either $b=T$, or $b+1$ must be an honest slot. Likewise, either $a=1$, or $a-1$ must be an honest slot. 
Let $F$ be a fork for $w_{1} \ldots w_{b}$ and let $u \in F, \ell(u)=a-1$ be an honest tine. (If $a=1$, we can take $u$ as the root vertex.) Let $t$ be a maximum-length honest tine in $F$ and assume that length $(t)=\operatorname{length}(u)+\#_{\mathrm{h}}(I)+\#_{\mathrm{H}}(I)$. Since $I$ is A-heavy, Fact 1 states that it is possible to augment $u$ into an adversarial extension $t^{\prime}$ viable at the onset of slot $b+1$. As $t^{\prime}$ will not contain any vertex from the honest slot $s, s$ does not have the bottleneck property in $w$.

The following theorem shows that a uniquely honest Catalan slot has the UVP.

Theorem 3. Let $w \in\{h, H, A\}^{T}$ be a characteristic string. Let $s \in[T]$ be a uniquely honest slot in $w$. Slot $s$ is Catalan in $w$ if and only if it has the UVP in $w$.

Proof. (The reverse implication.) Since $s$ has the UVP it satisfies the (weaker) bottleneck property. By Fact 3 the honest slot $s$ must be Catalan.

(The forward implication.) By assumption, slot $s$ has a unique honest leader. Let $\tau$ be the unique honest tine at slot $s$. By Fact 2 the honest tine $\tau$ is the only viable tine at the onset of slot $s+1$. If $s=T$ then $\tau$ is the only viable tine at the onset of slot $T+1$. Now suppose $s \leq T-1$. As $s$ is a Catalan slot, slots $s$ and $s+1$ must be honest. Let $t$ be a viable tine at the onset of some slot $k, k \geq s+2$. We claim that $\tau$ must be a prefix of $t$.

Suppose, for a contradiction, that $t$ does not contain $\tau$ as its prefix. Let $B_{1}$ be the last honest vertex on $t$ such that $\ell\left(B_{1}\right) \leq s-1$. (If $s=1$ or no such vertex can be found, take $B_{1}$ as the root vertex.) Likewise, let $B_{2}$ be the first honest vertex, if it exists, on $t$ such that $\ell\left(B_{2}\right) \in[s+1, k-1]$.

Suppose $B_{2}$ exists. If $\ell\left(B_{2}\right)=s+1$ then, by Observation $1 B_{2}$ builds on $\tau$, contradicting our assumption that $\tau$ is not a prefix of $t$. Otherwise, suppose $\ell\left(B_{2}\right) \in[s+2, k-1]$. Let $I$ be the interval $\left[\ell\left(B_{1}\right)+1, \ell\left(B_{2}\right)-1\right]$. Clearly, $I$ contains $s$. If $t$ contains any adversarial vertex between $B_{1}$ and $B_{2}$ then, by Corollary 1 I must be A-heavy; but this contradicts the assumption that $s$ is a Catalan slot. Otherwise, $B_{2}$ builds on top of $B_{1}$ and, in particular, $B_{1}$ must be viable at the onset of slot $\ell\left(B_{2}\right) \geq s+1$. Since $\ell(\tau)=s$, this means length $\left(B_{1}\right) \geq \operatorname{length}(\tau)$. However, $\operatorname{since}$ $\ell\left(B_{1}\right)<s$, by the monotonicity of the honest-depth function $\mathbf{d}(\cdot), \operatorname{length}(\tau) \geq 1+\operatorname{length}\left(B_{1}\right)$. This contradicts the inequality above.

Now suppose $B_{2}$ does not exist. We claim that $t$ is an adversarial tine. To see why, note that if $t$ were honest and $\ell(t) \geq s+1$ then there would have been a $B_{2}$. Since $s$ is a uniquely honest slot and $\tau$ is not a prefix of $t$ by assumption, $\ell(t) \neq s$ if $t$ is honest.

Finally, if $t$ is honest and $\ell(t) \leq s-1$ then, by Fact $2 t$ cannot be viable at the onset of slot $s+1$ since $s$ is Catalan. Since $s+1$ is an honest slot, honest tines with label $s+1$ will be strictly longer than $t$ and, therefore, $t$ cannot be viable at the onset of slot $k \geq s+2$ either. We conclude that $t$ must be an adversarial tine viable at the onset of slot $k$. By Fact 1 the interval $I=\left[\ell\left(B_{1}\right)+1, k-1\right]$ must be A-heavy. However, since $I$ contains $s$, it contradicts the fact that $s$ is a Catalan slot.

It follows that every viable tine $t \in F, \ell(t) \geq s+1$ must contain $\tau$ as its prefix.

The following theorem shows that under axiom $\mathbf{A 0 ^ { \prime }}$ two consecutive Catalan slots imply that the first slot has the UVP.

Theorem 4. Let $w \in\{\mathrm{H}, \mathrm{A}\}^{T}$ be a bivalent characteristic string and axiom $\left[\boldsymbol{A O}^{\prime}\right.$ is satisfied. Let $s \in[2, T]$ be an integer such that $s$ and $s-1$ are two honest slots in $w$. The following statements are equivalent: (i) Slots $s, s-1$ are Catalan. (ii) If $s \leq T-1$, both $s$ and $s-1$ have the UVP. Otherwise, slot $T-1$ has the UVP but slot $T$ has the bottleneck property.

Proof. Since the slots $s, s-1$ satisfy the (weaker) bottleneck property, Fact 3 implies that they must be Catalan slots. This proves (ii) implies (i)

Now let us prove that (i) implies (ii) Slots $s, s-1$ are Catalan. Let $V_{s}$ (resp. $V_{s+1}$ ) be the set of all viable tines at the onset of slot $s$ (resp. slot $s+1$ ). Since $s-1$ (resp. $s$ ) is a Catalan slots, we use Fact 2 and conclude that $V_{s}$ (resp. $V_{s+1}$ ) can contain only maximum-length honest tines $t, \ell(t)=s-1$ (resp. $\ell(t)=s$ ). Let $u_{s} \in V_{s}$ be the unique vertex determined by the consistent tie-breaking rule when applied to the set $V_{s}$. Define $u_{s+1} \in V_{s+1}$ in an analogous way for the set $V_{s+1}$.

Let $k \in[s+1, T+1]$ be an integer. We wish tho show that for every tine $t$ viable at the onset of slot $k$, the following holds: (i) if $s \leq T-1$ then $u_{s} \prec u_{s+1} \preceq t$, and (ii) if $s=T$ then $u_{T-1} \prec t$ where $\ell(t)=T$.

All tines at the honest slot $s$ build upon $u_{s}$. If $s=T$, we are done. Otherwise, i.e., if $s \leq T-1$, let $\tau=u_{s+1}$ and note that $u_{s} \prec u_{s+1}=\tau$. If $k=s+1$, we are done since by Fact2 every tine at the honest slot $k$ will build upon $\tau$. 
It remains to reason about the case $s \leq T-2$ and $k \geq s+2$. Consider a tine $t$ which is viable at the onset of slot $k$. (All we know about $t$ 's label is that $\ell(t) \leq k-1$.) We claim that $\tau \prec t$. Suppose, towards a contradiction, that $\tau$ is not a prefix of $t$. Let $B_{1}$ be the last honest vertex on $t$ such that $\ell\left(B_{1}\right) \leq s-1$. (If no such vertex can be found, take $B_{1}$ as the root vertex.) Likewise, let $B_{2}$ be the first honest vertex on $t$ such that $\ell\left(B_{2}\right) \in[s+1, k-1]$.

Below, we show that every choice for $B_{1}, B_{2}$ leads to a contradiction and, therefore, $\tau$ must be a prefix of $t$. If $B_{2}$ exists then, by construction, $\ell\left(B_{1}\right)<s<\ell\left(B_{2}\right) \leq k-1$. If $\ell\left(B_{2}\right)=s+1$ then, as we have argued earler, $B_{2}$ must have built on $\tau$. This contradicts our assumption that $\tau$ is not a prefix of $t$. Otherwise, suppose $\ell\left(B_{2}\right) \geq s+2$. Let $I$ be the interval $\left[\ell\left(B_{1}\right)+1, \ell\left(B_{2}\right)-1\right]$ and note that $I$ contains $s$. There can be two scenarios. If $t$ contains an adversarial vertex between $B_{1}$ and $B_{2}$ then, by Corollary 1 must be A-heavy; but this contradicts the assumption that $s$ is a Catatan slot. Otherwise, $B_{2}$ builds on top of $B_{1}$ and, in particular, $B_{1}$ must be viable at the onset of slot $\ell\left(B_{2}\right) \geq s+1$. Since $\ell(\tau)=s$, this means length $\left(B_{1}\right) \geq \operatorname{length}(\tau)$. However, since $\ell\left(B_{1}\right)<s$, by the monotonicity of the honest-depth function $\mathbf{d}(\cdot)$, length $(\tau) \geq 1+\operatorname{length}\left(B_{1}\right)$. This contradicts the inequality above.

If $B_{2}$ does not exist then we claim that $t$ is an adversarial tine. To see why, note that if $t$ were honest and $\ell(t) \geq$ $s+1$ then there would have been a $B_{2}$. If $t$ were honest with $\ell(t)=s, t \neq \tau$ then $t$ would not be viable at the onset of slot $s+2$. This is because $s$ is a Catalan slot and as such, each vertex from slot $s+1$ builds on $\tau, \operatorname{length}(\tau) \geq \operatorname{length}(t)$. Hence tines viable at the onset of slot $s+2$ must have length at least $1+\operatorname{length}(\tau)>\operatorname{length}(t)$. Finally, if $t$ is honest and $\ell(t) \leq s-1$ then, by Fact $2 t$ cannot be viable at the onset of slot $s+1$ since $s$ is Catalan. Since $s+1$ is an honest slot, honest tines with label $s+1$ will be strictly longer than $t$ and, therefore, $t$ cannot be viable at the onset of slot $k \geq s+2$ either. We conclude that $t$ must be an adversarial tine viable at the onset of slot $k$. By Fact 1 . the interval $I=\left[\ell\left(B_{1}\right)+1, k-1\right]$ must be A-heavy. However, since $I$ contains $s$, it contradicts the fact that $s$ is a Catalan slot.

\section{Main theorems via tail bounds for Catalan slots}

In the previous section, we explored the structural connection between the UVP and Catalan slots. In this section, we present two bounds on the stochastic event "Catalan slots are rare." Specifically, Bound 1 concerns uniquely honest Catalan slots and complements Theorem 3 Bound 2 concerns two consecutive Catalan slots and complements Theorem 4 We defer the proofs till the next section and prove the main theorems below.

Recall the $\left(\epsilon, p_{\mathrm{h}}\right)$-Bernoulli condition from 7

Bound 1. Let $T, s, k \in \mathbb{N}, T \geq s+k$ and $\epsilon, q_{\mathrm{h}} \in(0,1)$. Let $w$ be a characteristic string satisfying the $\left(\epsilon, q_{\mathrm{h}}\right)$-Bernoulli condition and let $y=w_{s} \ldots w_{s+k-1}$. Then

$$
\underset{w}{\operatorname{Pr}}[w \text { does not contain a uniquely honest Catalan slot in } y] \leq \exp \left(-k \cdot \Omega\left(\min \left(\epsilon^{3}, \epsilon^{2} q_{\mathrm{h}}\right)\right)\right) \text {. }
$$

In particular, when $q_{\mathrm{h}}=(1+\epsilon) / 2$, the bound above coincides with the bound in [3]; it follows that the current analysis subsumes their result.

Bound 2. Let $T, s, k \in \mathbb{N}, T \geq s+k$ and $\epsilon \in(0,1)$. Let $w$ be a bivalent characteristic string satisfying the $(\epsilon, 0)$ Bernoulli condition and let $y=w_{s} \ldots w_{s+k-1}$. Then

$$
\underset{w}{\operatorname{Pr}}[w \text { does not contain two consecutive Catalan slots in } y] \leq \exp \left(-k \cdot \Omega\left(\epsilon^{3}(1+O(\epsilon))\right)\right) \text {. }
$$

Proof of Theorem 1 We consider the distribution $\mathcal{B}$ first. Write $w=x y z,|x|=s-1$. Recall that $\mathbf{S}^{s, k}[\mathcal{B}]=$ $\operatorname{Pr}_{w \sim \mathcal{B}}[s$ is not $k$-settled in $w]$. Theorem 3] and Equation (1) implies that if $w$ contains a uniquely honest Catalan slot $c \in[s, s+k]$ then slot $s$ must be $k$-settled in $w$. In fact, by virtue of Fact 2 it suffices to take $c \in[s, s+k-1]$, i.e., $|x| \leq c \leq|x y|$. Thus the probability above is bounded by Bound 1 which renames $p_{\mathrm{h}}=q_{\mathrm{h}}$. This proves the first inequality.

Now let us prove the second inequality. For any player playing the settlement game, let $C$ be the set of strings on which the player wins. Clearly, $C$ is monotone with respect to the partial order $\leq$ defined on $\{\mathrm{h}, \mathrm{H}, \mathrm{A}\}^{T}$ (see below Definition 6). To see why, note that if the player wins on a specific string $w$, he can certainly win on any string $w^{\prime}$ so that $w \leq w^{\prime}$. By assumption, $\mathcal{W} \preceq \mathcal{B}$. It follows from Definition 6 that $\operatorname{Pr}_{\mathcal{W}}[w] \leq \operatorname{Pr}_{\mathcal{B}}[w]$ for any $w$ in the monotone 
set $C$. By referring to the definition of settlement insecurity (see Definition 5 , we conclude that $\mathbf{S}^{s, k}[\mathcal{W}] \leq \mathbf{S}^{s, k}[\mathcal{B}]$.

Proof of Theorem 2, This proof is identical to the proof of Theorem 1 except that we need to refer to Theorem 4 in lieu of Theorem 3 and Bound[2] in lieu of Bound[1

\section{Proofs of Bounds 1 and 2}

As a rule, we denote the probability distribution associated with a random variable using uppercase script letters. Observe that if $Y \preceq X$ and $Z$ is independent of both $X$ and $Y$, then $Z+Y \preceq Z+X$. In addition, for any non-decreasing function $u$ defined on $\Omega, Y \preceq X$ implies $u(Y) \leq u(X)$.

Generating functions. We reserve the term generating function to refer to an "ordinary" generating function which represents a sequence $a_{0}, a_{1}, \ldots$ of non-negative real numbers by the formal power series $\mathrm{A}(Z)=\sum_{t=0}^{\infty} a_{t} Z^{t}$. We denote the above correspondence as $\left\{a_{t}\right\} \longleftrightarrow \mathrm{A}(Z)$. When $\mathrm{A}(1)=\sum_{t} a_{t}=1$ we say that the generating function is a probability generating function; in this case, the generating function $\mathrm{A}$ can naturally be associated with the integer-valued random variable $A$ for which $\operatorname{Pr}[A=k]=a_{k}$. If the probability generating functions $\mathrm{A}$ and $\mathrm{B}$ are associated with the random variables $A$ and $B$, it is easy to check that $\mathrm{A} \cdot \mathrm{B}$ is the generating function associated with the convolution $A+B$ (where $A$ and $B$ are assumed to be independent). Translating the notion of stochastic dominance to the setting with generating functions, we say that the generating function $\mathrm{A}$ stochastically dominates $\mathrm{B}$ if $\sum_{t \leq T} a_{t} \leq \sum_{t \leq T} b_{t}$ for all $T \geq 0$; we write $\mathrm{B} \preceq \mathrm{A}$ to denote this state of affairs. If $\mathrm{B}_{1} \preceq \mathrm{A}_{1}$ and $\mathrm{B}_{2} \preceq \mathrm{A}_{2}$ then $\mathrm{B}_{1} \cdot \mathrm{B}_{2} \preceq \mathrm{A}_{1} \cdot \mathrm{A}_{2}$ and $\alpha \mathrm{B}_{1}+\beta \mathrm{B}_{2} \preceq \alpha \mathrm{A}_{1}+\beta \mathrm{A}_{2}$ (for any $\alpha, \beta \geq 0$ ). Moreover, if $\mathrm{B} \preceq \mathrm{A}$ then it can be checked that $\mathrm{B}(\mathrm{C}) \preceq \mathrm{A}(\mathrm{C})$ for any probability generating function $\mathrm{C}(Z)$, where we write $\mathrm{A}(\mathrm{C})$ to denote the composition $\mathrm{A}(\mathrm{C}(Z))$.

Finally, we remark that if $\mathrm{A}(Z)$ is a generating function which converges as a function of a complex $Z$ for $|Z|<R$ for some non-negative $R, R$ is called the radius of convergence of A. It follows from Theorem 2.19 in [12] that $\lim _{k \rightarrow \infty}\left|a_{k}\right| R^{k}=0$ and that $\left|a_{k}\right|=O\left(R^{-k}\right)$. In addition, if A is a probability generating function associated with the random variable $A$ then it follows that $\operatorname{Pr}[A \geq T]=O\left(R^{-T}\right)$.

\subsection{Proof of Bound 1}

Let $p=(1-\epsilon) / 2$ and $q=(1+\epsilon) / 2$ so that $q-p=\epsilon$. Let $q_{\mathrm{H}}=q-q_{\mathrm{n}}$. Let $B$ denote the event that $w$ does not contain a uniquely honest Catalan slot in $y$. We would like to bound $\operatorname{Pr}_{w}[B]$ from above.

Define the process $W=\left(W_{t}: t \in \mathbb{N}\right), W_{t} \in\{ \pm 1\}$ as $W_{t}=1$ if and only if $w_{t}=$ A. Let $S=\left(S_{t}: t \in \mathbb{N}\right), S_{t}=$ $\sum_{i \leq t} W_{i}$ be the position of the particle at time $t$. Thus $S$ is a random walk on $\mathbb{Z}$ with $\epsilon$ negative (i.e., downward) bias. By convention, set $W_{0}=S_{0}=0$.

Case 1: $x$ is an empty string. In this case, we write $w=y z$ so that $|y|=k$. Let $c_{t}$ be the probability that $t$ is the first uniquely honest Catalan slot in $w$ with $c_{0}=0$, and consider the probability generating function $\left\{c_{t}\right\} \longleftrightarrow$ $\mathrm{C}(Z)=\sum_{t=0}^{\infty} c_{t} Z^{t}$. Controlling the decay of the coefficients $c_{t}$ suffices to give a bound on $\operatorname{Pr}[B]$, i.e., the probability that $y$ does not contain a Catalan slot, because this probability is at most $1-\sum_{t=0}^{k-1} c_{t}=\sum_{t=k}^{\infty} c_{t}$. To this end, we develop a closed-form expression for a related probability generating function $\hat{\mathrm{C}}(Z)=\sum_{t} \hat{c}_{t} Z^{t}$ which stochastically dominates $\mathrm{C}(Z)$. Recall that this means that for any $k, \sum_{t \geq k} c_{k} \leq \sum_{t \geq k} \hat{c}_{k}$. Finally, bound the latter sum by using the analytic properties of $\hat{\mathrm{C}}(Z)$.

Treating the random variables $W_{1}, \ldots$ as defining a (negatively) biased random walk, define $\mathrm{D}$ (resp. A) to be the generating function for the descent stopping time (resp. the ascent stopping time) of the walk; this is the first time the random walk, starting at 0 , visits -1 (resp. +1 ). The natural recursive formulation of these descent time yield simple algebraic equations for the descent generating function, $\mathrm{D}(Z)=q Z+p Z \mathrm{D}(Z)^{2}$ and $\mathrm{A}(Z)=p Z+q Z \mathrm{~A}(Z)^{2}$, and 
from this we may conclude

$$
\begin{aligned}
& \mathrm{D}(Z)=\left(1-\sqrt{1-4 p q Z^{2}}\right) / 2 p Z, \\
& \mathrm{~A}(Z)=\left(1-\sqrt{1-4 p q Z^{2}}\right) / 2 q Z .
\end{aligned}
$$

Note that while $D$ is a probability generating function, $A$ is not: according to the classical "gambler's ruin" analysis, the probability that a negatively-biased random walk starting at 0 ever rises to 1 is exactly $p / q$; thus $\mathrm{A}(1)=p / q$.

Recall that a slot is Catalan in $w$ if and only if it is both left-Catalan and right-Catalan. A slot is left-Catalan if the walk $S$ descends to a new low at that slot. In addition, the same slot (say $s$ ) is right-Catalan if the walk never reaches to that level in future, i.e., $S_{s} \geq S_{i}, i \geq s+1$. The probability of this event is $1-\mathrm{A}(1)=1-p / q=\epsilon / q$, conditioned on the fact that $W_{s}=-1$.

Assume that the walk is now at its historical minimum. (It may or may not be a new minimum.) We can think of the generating function $\mathrm{C}(Z)$ as a search procedure for finding the first uniquely honest Catalan slot. Let $v$ be the first symbol we observe. Let $\mathrm{E}(Z)$ be the generating function for a walk which makes an ascent with certainty and then descends again to its historical minimum. We claim that

$$
\begin{aligned}
\mathrm{C}(Z) & =p Z \mathrm{D}(Z) \mathrm{C}(Z)+q_{\mathrm{h}} Z \cdot \epsilon / q+q_{\mathrm{h}} Z \cdot p / q \cdot \mathrm{E}(Z) \mathrm{C}(Z)+q_{\mathrm{H}} Z \mathrm{C}(Z) \\
& =\frac{\left(q_{\mathrm{h}} \epsilon / q\right) Z}{1-\left(p Z \mathrm{D}(Z)+\left(q_{\mathrm{h}} p / q\right) Z \mathrm{E}(Z)+q_{\mathrm{H}} Z\right)} .
\end{aligned}
$$

Here is the explanation. Regarding the value of $v$, there can be four alternatives for the walk which is currently at its historical minimum:

(i) With probability $p$, we have $v=\mathrm{A}$ and the walk moves up. Then we wait till the walk makes a first descent and restart.

(ii) With probability $q_{\mathrm{h}} \cdot \epsilon / q$, we have $v=\mathrm{h}$ and the walk diverges below. Hence our search has succeeded and we stop.

(iii) With probability $q_{\mathrm{h}} \cdot(1-\epsilon / q)=q_{\mathrm{h}} p / q$, we have $v=\mathrm{h}$ and the walk returns to the origin from below. Then we wait for the walk to match its minimum again before we can restart. Note that $\mathrm{E}(Z)$ is the generating function for this "guaranteed ascent then match minimum" walk.

(iv) With probability $q_{\mathrm{H}}$, we have $v=\mathrm{H}$ and the walk moves down. Since we will reach a new minimum, we restart.

Since $\mathrm{E}(1)=1$ by assumption, $p+\left(q_{\mathrm{h}} p / q\right)+q_{\mathrm{H}}=1-q_{\mathrm{h}}(1-p / q)=1-q_{\mathrm{h}} \epsilon / q$. It follows that $\mathrm{C}(1)=\left(q_{\mathrm{h}} \epsilon / q\right) /(1-$ $\left.\left(1-q_{\mathrm{h}} \epsilon / q\right)\right)=1$; hence $\mathrm{C}(Z)$ is a probability generating function.

Instead of working directly with $\mathrm{E}(Z)$, we can work with a generating function $\hat{\mathrm{E}}(Z)$ which is identical to $\mathrm{E}(Z)$ for the initial ascending part but differs in the descending part. Specifically, in the descending part, the walk represented by $\hat{\mathrm{E}}(Z)$ descends as many levels as the number of steps it took to return to the origin. Clearly, $\mathrm{E}(Z) \preceq$ $\hat{\mathrm{E}}(Z) \triangleq \mathrm{A}(Z \mathrm{D}(Z)) / \mathrm{A}(1)$. Here, an individual term in $\mathrm{A}(Z \mathrm{D}(Z))=\sum_{i} a_{i} Z^{i} \mathrm{D}(Z)^{i}$ has the interpretation "if the first ascent took $i$ steps then immediately descend $i$ levels." Since $\mathrm{A}(Z)$ is not a probability generating function, we have to normalize it by $\mathrm{A}(1)$ to make sure that the ascent happens with certainty. Writing

$$
\mathrm{F}(Z) \triangleq p Z \mathrm{D}(Z)+q_{\mathrm{h}} Z \mathrm{~A}(Z \mathrm{D}(Z))+q_{\mathrm{H}} Z,
$$

note that

$$
\mathrm{C}(Z) \preceq \hat{\mathrm{C}}(Z) \triangleq\left(q_{\mathrm{h}} \epsilon / q\right) Z /(1-\mathrm{F}(Z)) .
$$

Since $\mathrm{F}(1)=p+q_{\mathrm{h}} p / q+q_{\mathrm{H}}=1-q_{\mathrm{h}}(1-p / q)=1-q_{\mathrm{h}} \epsilon / q$, we have $\hat{\mathrm{C}}(1)=1$, i.e., $\hat{\mathrm{C}}(Z)$ is a probability generating function. It remains to establish a bound on the radius of convergence of $\hat{C}$. A sufficient condition for the convergence of $\hat{\mathrm{C}}(z)$ for some $z \in \mathbb{R}$ is that all generating functions appearing in the definition of $\hat{\mathrm{C}}(z)$ converge at $z$ and that $\mathrm{F}(z) \neq 1$. 
The generating functions $\mathrm{D}(z)$ and $\mathrm{A}(z)$ converge when the discriminant $1-4 p q z^{2}$ is positive; equivalently $|z|<1 / \sqrt{1-\epsilon^{2}}=1+\epsilon^{2} / 2+O\left(\epsilon^{4}\right)$. In addition, conditioned on the convergence of $\mathrm{A}(z)$ and $\mathrm{D}(z)$, we can check that

$$
\mathrm{A}(z)<1 / 2 q z \text { and } \mathrm{D}(z)<1 / 2 p z .
$$

On the other hand, the convergence of $\mathrm{F}(z)$ depends on the convergence of $\mathrm{D}(z)$ and $\mathrm{A}(z \mathrm{D}(z))$. The convergence of $\mathrm{A}(z \mathrm{D}(z))$ is likewise determined by the positivity of its discriminant, i.e.,

$$
1-\left(1-\epsilon^{2}\right)\left(z \cdot \frac{1-\sqrt{1-\left(1-\epsilon^{2}\right) z^{2}}}{(1-\epsilon) z}\right)^{2}>0 .
$$

The inequality above implies that if $\mathrm{A}(z \mathrm{D}(z))$ converges when

$$
|z|<R_{1} \triangleq\left(\left(2 / \sqrt{1-\epsilon^{2}}-1 /(1+\epsilon)\right) /(1+\epsilon)\right)^{1 / 2},
$$

where

$$
R_{1}=1+\epsilon^{3} / 2+O\left(\epsilon^{4}\right) \approx \exp \left(\epsilon^{3}(1+O(\epsilon)) / 2\right) .
$$

Note that the radius of convergence of $\mathrm{A}(Z \mathrm{D}(Z))$ is smaller than that of $\mathrm{A}(Z)$ or $\mathrm{D}(Z)$.

We can check that when $\mathrm{F}(z)$ converges, it satisfies

$$
\mathrm{F}(z) \leq \mathrm{F}(|z|) .
$$

The claim is trivial for $z=0$. Otherwise, note that $\mathrm{D}(z)$ is an odd function and hence, $z \mathrm{D}(z)=|z| \mathrm{D}(|z|)$. Thus, for the claim to hold, we need only show that $z\left(q_{\mathrm{h}} \mathrm{A}(z \mathrm{D}(z))+q_{\mathrm{H}}\right) \leq|z|\left(q_{\mathrm{h}} \mathrm{A}(|z| \mathrm{D}(|z|))+q_{\mathrm{H}}\right)$. But the right-hand side equals $|z|\left(q_{\mathrm{h}} \mathrm{A}(z \mathrm{D}(z))+q_{\mathrm{H}}\right)$ and $\mathrm{A}(x)>0$ for real $x>0$, we can divide both sides by $q_{\mathrm{h}} \mathrm{A}(z \mathrm{D}(z))+q_{\mathrm{H}}$. The reduced inequality becomes $z /|z| \leq 1$. However, $z /|z|= \pm 1$ for any non-zero real $z$. Therefore, it suffices for us to require that $F(z) \neq 1$ for $z>0$.

We can also check that

$$
F(z) \text { is convex and increasing for } z \in\left[0, R_{1}\right) \text {. }
$$

To see why, note that since $z^{2}$ is convex in $z,\left(1-4 p q z^{2}\right)$ is concave. Since square root is non-decreasing and convex for positive $z, \sqrt{1-4 p q z^{2}}$ is concave and consequently, $-\sqrt{1-4 p q z^{2}}$ is convex. Since $1 / z^{2}$ is convex, it follows that $\mathrm{D}(z)$ and, by a similar reasoning, $\mathrm{A}(z)$ are convex. Next, observe that $\mathrm{A}(z \mathrm{D}(z))$ converges for $z \in\left[0, R_{1}\right)$ and hence it is also convex in $z$. Thus $\mathrm{F}(z)$ turns out to be a convex combination of convex functions; it follows that $F(z)$ is convex for $z \in\left(0, R_{1}\right)$. In addition, since $\mathrm{F}(0)=0$ and $\mathrm{F}(1)>0, \mathrm{~F}(z)$ must be increasing as well.

Let

$$
R_{2} \text { be the solution to the equation } \mathrm{F}(z)=1, z>0 .
$$

Then $\hat{\mathrm{C}}(z)$ would converge for $|z|<R \triangleq \min \left(R_{1}, R_{2}\right)$. It remains to characterize $R_{2}$ in terms of $\epsilon$ and $q_{\mathrm{h}}$. Note that $R_{1}<2$ as long as $\epsilon \leq 0.97$. Since the final bounds will be only asymptotic in $\epsilon$, it suffices for us to consider small $\epsilon$. That is to say, we consider the case where $0<z<R_{1}<2$, i.e., $z-1<1$.

If we express $\mathrm{F}(z)$ as its power series around $z=1$, we can check that

$$
\begin{aligned}
\mathrm{F}(1) & =1-\epsilon q_{\mathrm{h}} / q, \\
\mathrm{~F}^{\prime \prime}(1) & =\frac{1-\epsilon}{\epsilon^{5}}\left(q_{\mathrm{h}}(1+3 \epsilon)+q_{\mathrm{H}} \epsilon^{2}\right), \quad \text { and } \\
\mathrm{F}^{\prime}(1) & =p(1+1 / \epsilon)+q_{\mathrm{h}}(p / q)(1+(1+1 / \epsilon) / \epsilon)+q_{\mathrm{H}} .
\end{aligned}
$$

Since $\mathrm{F}^{\prime \prime}(1)>0$ and $\mathrm{F}(z)$ is convex and increasing, the first-order approximation

$$
f(z)=\left(1-\epsilon q_{\mathrm{h}} / q\right)+\mathrm{F}^{\prime}(1)(z-1)
$$


is a lower bound for $\mathrm{F}(z)$ when $1 \leq z<R_{1}$. The approximation error at any $z \in(1,2)$ is $\mathrm{F}(z)-f(z)=O(h(z))$ where we define

$$
h(z) \triangleq \mathrm{F}^{\prime \prime}(1)(z-1)^{2}
$$

Since the bounds we develop will have either $O(\cdot)$ or $\Omega(\cdot)$ in the exponent, it suffices to ensure that $R_{2}=\Theta\left(R_{2}^{*}\right)$. In the exposition below, we will only develop approximations $R_{2}^{*}$ satisfying $R_{2}=(1-\theta) R_{2}^{*}$ for a small positive constant $\theta \in(0,1)$.

In the special case $q_{\mathrm{H}}=0, \mathrm{~F}(Z)$ simplifies as $\mathrm{F}(Z)=p Z \mathrm{D}(Z)+q Z \mathrm{~A}(Z \mathrm{D}(Z))$. Note that $\mathrm{F}(Z)$ converges when $\mathrm{A}(Z \mathrm{D}(Z))$ does and it is not hard to check that $\mathrm{F}(z)<1$. Specifically, we know that $\mathrm{F}(z)$ converges when $z \in\left[0, R_{1}\right)$ and when it does, we claim that $\mathrm{F}(z)<1$. Specifically, when $z \in[0,1], \mathrm{F}(z) \leq \mathrm{F}(1)=1-\epsilon q_{\mathrm{h}} / q=1-\epsilon<1$ since $\epsilon<1$. On the other hand, we can check that $\mathrm{D}(z)$ is convex for $z \geq 0$ and, in particular, the first order approximation $1+(z-1) / \epsilon$ around $z=1$ is a lower bound for $\mathrm{D}(z), z \geq 1$. It follows that $\mathrm{D}(z) \geq 1$ for $z \in\left[1, R_{1}\right)$. Consequently, $\mathrm{F}(z) \leq p Z \mathrm{D}(Z)+q z \mathrm{~A}(z \mathrm{D}(z)) \cdot \mathrm{D}(z)=p z \mathrm{D}(z)+q x \mathrm{~A}(x)<1 / 2+1 / 2=1$ where we write $x=z \mathrm{D}(z)$ and use (4). Thus the radius of convergence of $\hat{\mathrm{C}}$ is $R_{1}$ if $q_{\mathrm{H}}=0$.

The remainder of the exposition considers the general case $0<q_{\mathrm{h}}<q$. Let the solution to the equation $f(z)=1$ be denoted by

$$
R_{2}^{*} \triangleq 1+\epsilon\left(q_{\mathrm{h}} / q\right) / \mathrm{F}^{\prime}(1)
$$

If $q_{\mathrm{h}}$ is small, $q=(1+\epsilon) / 2, p+\epsilon=q$ and $p / q^{3} \in[1,4]$, we can check that

$$
h\left(R_{2}^{*}\right)=O\left(\frac{p q}{\epsilon^{3}} \cdot\left(\frac{\epsilon^{2} q_{h} / q}{p(1+\epsilon)+\epsilon q}\right)^{2}\right)=O\left(\frac{\epsilon q_{\mathrm{h}}^{2} \cdot p q}{q^{2}(p+\epsilon)^{2}}\right)=O\left(\frac{\epsilon q_{\mathrm{h}}^{2} \cdot p}{q^{3}}\right)=O\left(\epsilon q_{\mathrm{h}}^{2}\right)
$$

i.e., it vanishes. Thus $f(z)$ is a good approximation for $\mathrm{F}(z)$. It follows that $\mathrm{F}^{\prime}(1) \approx p(1+1 / \epsilon)+q=q / \epsilon$ and, therefore,

$$
R_{2}^{*} \approx 1+\left(\epsilon q_{\mathrm{h}} / q\right) /(q / \epsilon)=1+q_{\mathrm{h}}(\epsilon / q)^{2} \approx \exp \left(\epsilon^{2} q_{\mathrm{h}} / q^{2}\right)=e^{O\left(\epsilon^{2} q_{\mathrm{h}}\right)}
$$

since $q \in(1 / 2,1)$. (Although we have an asymptotic notation, it is important that we have the right exponent on $\left.q_{\mathrm{h} .}\right)$

If, on the contrary, $q_{\mathrm{h}}=O(1)$ but $\epsilon$ vanishes then $\mathrm{F}^{\prime}(1)$ will be dominated by its second term; that is to say, $\mathrm{F}^{\prime}(1) \approx q_{\mathrm{h}}(p / q)(1+(1+1 / \epsilon) / \epsilon)=O\left(q_{\mathrm{h}} / \epsilon^{2}\right)$ and, therefore,

$$
R_{2}^{*} \approx 1+O\left(\left(\epsilon q_{\mathrm{h}} / q\right) /\left(q_{\mathrm{h}} / \epsilon^{2}\right)\right)=1+O\left(\epsilon^{3}\right)=e^{O\left(\epsilon^{3}\right)}
$$

since $q \approx 1 / 2$.

Recall that $R_{1}=\exp \left(O\left(\epsilon^{3}(1+O(\epsilon))\right)\right)$. It follows that $\hat{\mathrm{C}}(z)$ converges for $|z|$ less than

$$
R=\exp \left(O\left(\min \left(\epsilon^{3}, \epsilon^{2} q_{\mathrm{h}}\right)\right)\right) .
$$

Recall that if the radius of convergence of $\hat{C}$ is $\exp (\delta)$ then $\hat{c}_{k}=O\left(e^{-\delta k}\right)$. Hence, $\operatorname{Pr}[B]$ is a geometric sum and it is at most $O\left(e^{-\delta k}\right)$ as well. We conclude that

$$
\underset{w}{\operatorname{Pr}}[B] \leq O\left(e^{-k \ln R}\right)=\exp \left(-k \cdot \Omega\left(\min \left(\epsilon^{3}, \epsilon^{2} q_{\mathrm{h}}\right)\right)\right) .
$$

Case 2: $x$ is non-empty. Next, let us consider the case when $x \neq \varepsilon$, i.e., $|x| \geq 1$. Let $m=|x|$ and write $w=x y z$ where $|y|=k$. Recall the processes $\left(W_{t}\right)$ and $\left(S_{t}\right)$ defined on $w$ and, in addition, define $M=\left(M_{t}: t \in \mathbb{N}\right), M_{t}=$ $\min _{0 \leq i \leq t} S_{i}$ and $X=\left(X_{t}: t \in \mathbb{N}\right), X_{t}=S_{t}-M_{t}$. By convention, set $M_{0}=X_{0}=0$. Thus $X_{t}$ denotes the height of the walk $S$, at time $t$, with respect to its minimum $M_{t}$.

For a fixed value $h=X_{m}$, the relevant generating function would be $\mathrm{D}(Z)^{h} \hat{\mathrm{C}}$. Hence the final generating function we seek is

$$
\tilde{\mathrm{C}}(Z) \triangleq \sum_{h=0}^{\infty} \operatorname{Pr}\left[X_{m}=h\right] \cdot \mathrm{D}(Z)^{h} \hat{\mathrm{C}}(Z)
$$

whose $t$ th coefficient is the probability that $t$ is a Catalan slot in $y$. 
Note that $X=\left(X_{t}\right)$ is an $\epsilon$-downward biased random walk on non-negative integers with a reflective barrier at -1 . Specifically, for any $h \in \mathbb{N}, \operatorname{Pr}\left[X_{t}=h \mid X_{t-1}=h-1\right]=p$ and $\operatorname{Pr}\left[X_{t}=h-1 \mid X_{t-1}=h\right]=\operatorname{Pr}\left[X_{t}=\right.$ $\left.0 \mid X_{t-1}=0\right]=q$. In [4, Lemma 6.1], it is proved that the distribution of $X_{m}$ is stochastically dominated by the distribution of $X_{\infty}$, written $\mathcal{X}_{\infty}$ and defined, for $k=0,1,2, \ldots$, as

$$
\mathcal{X}_{\infty}(k)=\operatorname{Pr}\left[X_{\infty}=k\right] \triangleq\left(\frac{2 \epsilon}{1+\epsilon}\right) \cdot\left(\frac{1-\epsilon}{1+\epsilon}\right)^{k}=(1-\beta) \beta^{k}
$$

where $\beta \triangleq(1-\epsilon) /(1+\epsilon)$. Let

$$
\left\{\mathcal{X}_{\infty}(k)\right\} \longleftrightarrow \mathbf{X}_{\infty}(Z)=\frac{1-\beta}{1-\beta Z}
$$

It follows that $\tilde{\mathrm{C}}(Z)$ is dominated by

$$
\sum_{h=0}^{\infty} \mathcal{X}_{\infty}(h) \mathrm{D}(Z)^{h} \hat{\mathrm{C}}(Z)=\mathrm{X}_{\infty}(\mathrm{D}(Z)) \hat{\mathrm{C}}(Z)=\frac{(1-\beta) \hat{\mathrm{C}}(Z)}{1-\beta \mathrm{D}(Z)} .
$$

Let $\star$ denote the quantity above. For it to converge, we need to check that $\mathrm{D}(Z)$ should never converge to $1 / \beta$. Since the radius of convergence of $\mathrm{D}(Z)$-which is $\left(1-\epsilon^{2}\right)^{-1 / 2}$-is strictly less than $(1+\epsilon) /(1-\epsilon)$ for $\epsilon>0$, we conclude that $\star$ converges if both $\mathrm{D}(Z)$ and $\hat{\mathrm{C}}(Z)$ converge. The radius of convergence of $\star$ would be the smaller of the radii of convergence of $\mathrm{D}(Z)$ and $\hat{\mathrm{C}}(Z)$. We already know from the previous analysis that $\hat{\mathrm{C}}(Z)$ has the smaller radius of convergence of these two; therefore, the bound on $\operatorname{Pr}_{w}[B]$ from the previous case holds for $|x| \geq 0$.

\subsection{Proof of Bound 2}

Let $p=(1-\epsilon) / 2$ and $q=1-p$; thus $q-p=\epsilon$. Let $B$ denote the event that $w$ does not contain two consecutive Catalan slots in $y$. We would like to bound $\operatorname{Pr}_{w}[B]$ from above.

Define the process $W=\left(W_{t}: t \in \mathbb{N}\right), W_{t} \in\{ \pm 1\}$ as $W_{t}=1$ if and only if $w_{t}=$ A. Let $S=\left(S_{t}: t \in \mathbb{N}\right), S_{t}=$ $\sum_{i \leq t} W_{i}$ be the position of the particle at time $t$. Thus $S$ is a random walk on $\mathbb{Z}$ with $\epsilon$ negative (i.e., downward) bias. By convention, set $W_{0}=S_{0}=0$.

Case 1: $x$ is an empty string. In this case, we write $w=y z$ so that $|y|=k$. Let $m_{t}$ denote the probability that $t$ is the first index so that both $t$ and $t+1$ are Catalan slots in $w$, with $m_{0}=0$, and consider the probability generating function $\left\{m_{t}\right\} \longleftrightarrow \mathrm{M}(Z)=\sum_{t=0}^{\infty} m_{t} Z^{t}$. Controlling the decay of the coefficients $m_{t}$ suffices to give a bound on $\operatorname{Pr}[B]$, i.e., the probability that $y$ does not contain two consecutive Catalan slots, because this probability is at most $1-\sum_{t=0}^{k-1} m_{t}=\sum_{t=k}^{\infty} m_{t}$. To this end, we develop a closed-form expression for a related probability generating function $\hat{\mathrm{M}}(Z)=\sum_{t} \hat{m}_{t} Z^{t}$ which stochastically dominates $\mathrm{M}(Z)$. Recall that this means that for any $k, \sum_{t \geq k} m_{k} \leq \sum_{t \geq k} \hat{m}_{k}$. Finally, bound the latter sum by using the analytic properties of $\hat{\mathrm{M}}(Z)$.

Recall the "first ascent" and "first descent" generating functions $\mathrm{A}(Z)$ and $\mathrm{D}(Z)$ from the proof of Bound 1. We wish to devise the generating function for the first occurrence of a left-Catalan slot immediately followed by a rightCatalan slot. To that end, note that $\mathrm{D}(Z)$ is the generating function for the first left-Catalan slot. The generating function for the first right-Catalan slot can be devised as follows. Consider the walk $S$ starting at the origin. With probability $q(1-p / q)=\epsilon$, the walk will immediately descend a step and never return to the origin. But this means $S_{1} \leq S_{t}, t \geq 2$ and hence the first slot is a right-Catalan slot and we are done. Otherwise, i.e., with probability $1-\epsilon$, the walk makes a (guaranteed) return to the origin in future. In this case, we will have to restart our search for the next consecutive Catalan slots but, before that, we will have to ensure that we are in a "safe position." In particular, we can safely restart our search if Specifically, if the current position (i.e., level) of the walk is at its historical minimum, we can restart our search by applying $\mathrm{D}(Z)$ to find the next left-Catalan slot. Thus an "epoch" begins with a guaranteed return and ends when the walk descends to a new level for the first time. $\operatorname{Let} \mathrm{E}(Z)$ be the 
generating function of an epoch. Thus we can write

$$
\begin{aligned}
\mathrm{M}(Z) & =\mathrm{D}(Z) \cdot\{\epsilon+(1-\epsilon) \mathrm{E}(Z) \mathrm{M}(Z)\} \\
& =\frac{\epsilon \mathrm{D}(Z)}{1-(1-\epsilon) \mathrm{E}(Z)} .
\end{aligned}
$$

An epoch can have two shapes. If an epoch starts with an up-step (i.e., an "up" shape), it is easy to see that the epoch ends as soon as the walk returns to the origin from above and, importantly, that the walk will (eventually) return to the origin with probability one. However, if the epoch starts with a down-step (i.e., a "down" shape), we have to "remember" the lowest level $\ell$ touched by the walk in its way to its (sure) ascent to the origin and then descend $\ell$ levels to end the epoch. In particular, we have to ensure that we return to the origin with probability one.

A generating function of a stopping time of a random walk is ill suited to "remember" its historical minimum/maximum. However, it can remember the length of the walk for free. Thus, instead of working directly with $\mathrm{E}(Z)$, we work with a generating function $\hat{\mathrm{E}}(Z)$ which is identical to $\mathrm{E}(Z)$ for the up shape but differs in the down shape. Specifically, in the down shape, the walk represented by $\hat{E}(Z)$ descends as many levels as the number of steps it took to return to the origin. Clearly, $\mathrm{E} \preceq \hat{E}$ where

$$
\hat{\mathrm{E}}(Z) \triangleq p Z \mathrm{D}(Z)+q Z \mathrm{~A}(Z \mathrm{D}(Z)) / \mathrm{A}(1) .
$$

Here, the first term denotes the "return to origin from above" shape. An individual term in $\mathrm{A}(Z \mathrm{D}(Z))=\sum_{t} a_{t} Z^{t} \mathrm{D}(Z)^{t}$ has the interpretation "if the first ascent took $t$ steps then follow it by descending $t$ levels." Since $A(Z)$ is not a probability generating function, we have to normalize it by $\mathrm{A}(1)$ to denote that the ascent happens with certainty. This implies,

$$
\mathrm{M}(Z) \preceq \hat{\mathrm{M}}(Z) \triangleq \frac{\epsilon \mathrm{D}(Z)}{1-(1-\epsilon) \hat{\mathrm{E}}(Z)}
$$

It remains to establish a bound on the radius of convergence of $\hat{\mathrm{M}}$. A sufficient condition for the convergence of $\hat{\mathrm{M}}(z)$ for some $z \in \mathbb{R}$ is that all generating functions appearing in the definition of $\hat{\mathrm{M}}$ converge at $z$ and that $(1-\epsilon) \hat{\mathrm{E}}(Z) \neq 1$.

By retracing our footsteps as in the proof of Bound 1 we can see that $\mathrm{D}(z), \mathrm{A}(z)$, and $\mathrm{A}(z \mathrm{D}(z))$ converge when $|z|$ satisfies (5). Moreover, since $\mathrm{D}(Z)$ is a probability generating function, it follows that $\hat{\mathrm{E}}(Z)$ is stochastically dominated by $p Z \mathrm{D}(Z)+q Z \mathrm{~A}(Z \mathrm{D}(Z)) / \mathrm{A}(1) \cdot \mathrm{D}(Z)$. Therefore, when $\hat{\mathrm{E}}(z)$ converges for some $z$, it satisfies

$$
\begin{aligned}
\hat{\mathrm{E}}(z) & \leq p z \mathrm{D}(z)+(q / p)(q z \mathrm{D}(z)) \mathrm{A}(z \mathrm{D}(z)) \\
& <1 / 2+(q / p) / 2
\end{aligned}
$$

since $\mathrm{A}(1)=p / q, p z \mathrm{D}(z)<1 / 2$, and $q x \mathrm{~A}(x)<1 / 2$ for any $z, x$ so that $\mathrm{A}(x)$ and $\mathrm{D}(z)$ converge, respectively. Therefore, $(1-\epsilon) \hat{\mathrm{E}}(z)=2 p \hat{\mathrm{E}}(z)<p+q=1$. It follows that $\hat{\mathrm{M}}(z)$ converges for $|z|<1+\epsilon^{3} / 2+O\left(\epsilon^{4}\right) \leq$ $\exp \left(\epsilon^{3} / 2+O\left(\epsilon^{4}\right)\right)$. Recall that if the radius of convergence of $\hat{\mathrm{M}}$ is $\exp (\delta)$ then $\operatorname{Pr}[B]$ is $O(1) \cdot e^{-\delta k}$. We conclude that

$$
\operatorname{Pr}_{w}[B] \leq O(1) \cdot e^{-\epsilon^{3}(1+O(\epsilon)) k / 2}
$$

Case 2: $x$ is non-empty. This part of the proof is the same as the $|x| \geq 1$ case in the proof of Bound 1 The only difference is that $\hat{\mathrm{C}}(Z)$ and $\tilde{\mathrm{C}}(Z)$ would be replaced by $\hat{\mathrm{M}}(Z)$ and $\tilde{\mathrm{M}}(Z)$, respectively, where

$$
\tilde{\mathrm{M}}(Z) \preceq \sum_{h=0}^{\infty} \mathcal{X}_{\infty}(h) \mathrm{D}(Z)^{h} \hat{\mathrm{M}}(Z) .
$$

We conclude that the bound in (11) holds when $|x| \geq 0$. 


\section{An optimal online adversary against slot settlement}

In this section, we introduce additional elements of the fork framework from Blum et al. [3], most notably the notions of "reach" and "relative margin." We show that relative margin is just as expressive as the Catalan slots for characterizing slot settlement. Next, we prove a recurrence relation for relative margin; it can be used to compute the probability that a given slot is $k$-settled, when the symbols of the characteristic string are i.i.d . Finally, we present an adversary who, given a characteristic string one symbol at a time, optimally attacks the settlement of all slots at once.

\subsection{Closed forks, reach, and extensions}

Definition 12 (Closed fork). A fork $F$ is closed if every leaf is honest. For convenience, we say the trivial fork is closed.

Closed forks have two nice properties that make them especially useful in reasoning about the view of honest parties. First, all honest observers will select a unique longest tine from this fork (since all longest tines in a closed fork are honest, honest parties are aware of all previous honest blocks, they observe the longest chain rule, and they employ the same consistent tie-breaking rule). Second, closed forks intuitively capture decision points for the adversary. The adversary can potentially show many tines to many honest parties, but once an honest node has been placed on top of a tine, any adversarial blocks beneath it are part of the public record and are visible to all honest parties. For these reasons, we will often find it easier to reason about closed forks than arbitrary forks.

The next few definitions are the start of a general toolkit for reasoning about an adversary's capacity to build highly diverging paths in forks, based on the underlying characteristic string.

Definition 13 (Gap, reserve, and reach). For a closed fork $F \vdash w$ and its unique longest tine $\hat{t}$, we define the gap of a tine $t$ to be $\operatorname{gap}(t)=\operatorname{length}(\hat{t})-\operatorname{length}(t)$. Furthermore, we define the reserve of $t$, denoted reserve $(t)$, to be the number of adversarial indices in $w$ that appear after the terminating vertex of $t$. More precisely, if $v$ is the last vertex of $t$, then

$$
\text { reserve }(t)=\mid\left\{i \mid w_{i}=1 \text { and } i>\ell(v)\right\} \mid .
$$

These quantities together define the reach of a tine: $\operatorname{reach}(t)=\operatorname{reserve}(t)-\operatorname{gap}(t)$.

The notion of reach can be intuitively understood as a measure of the resources available to our adversary in the settlement game. Reserve tracks the number of slots in which the adversary has the right to issue new blocks. When reserve exceeds gap (or equivalently, when reach is nonnegative), such a tine could be extended-using a sequence of dishonest blocks-until it is as long as the longest tine. Such a tine could be offered to an honest player who would prefer it over, e.g., the current longest tine in the fork. In contrast, a tine with negative reach is too far behind to be directly useful to the adversary at that time.

Definition 14 (Maximum reach). For a closed fork $F \vdash w$, we define $\rho(F)$ to be the largest reach attained by any tine of $F$, i.e.,

$$
\rho(F)=\max _{t} \operatorname{reach}(t) .
$$

Note that $\rho(F)$ is never negative (as the longest tine of any fork always has reach at least 0 ). We overload this notation to denote the maximum reach over all forks for a given characteristic string:

$$
\rho(w)=\max _{\substack{F \vdash w \\ F \text { closed }}}\left[\max _{t} \operatorname{reach}(t)\right] .
$$

Reach of vertices is always non-increasing as we move down a tine. That is, if $B_{1}, B_{2}, \ldots$ are vertices on the same tine in the root-to-leaf order, then reach $\left(B_{i}\right) \leq \operatorname{reach}\left(B_{i+1}\right)$. The inequality is strict if $B_{i+1}$ is honest. Consequently, the reach of an adversarial tine is no more than the reach of the last honest vertex in that tine. In any fork, the reach of a maximum-length tine is always non-negative. Hence, an honest tine with the maximum length over all honest tines will always have a non-negative reach. Thanks to the monotonicity of the honest-depth function $\mathbf{d}(\cdot)$, if there are multiple honest tines having the (same) maximum length among all honest tines, they must have the same label. Therefore, if $h$ is the last honest slot in $w$ and $t$ a maximum-length honest tine with label $h$, then $\operatorname{reach}(t) \geq 0$. 
Non-negative reach, A-heaviness, and viable adversarial extensions. Let $w \in\{\mathrm{h}, \mathrm{H}, \mathrm{A}\}^{T}, s \in[T+1]$, and $F \vdash w_{1} \ldots w_{s-1}$ an arbitrary fork. Let $B \in F$ be an honest vertex and $t$ a maximum-length tine in $F$. Consider the following statements:

(a) $B$ has an adversarial extension viable at the onset of slot $s$.

(b) $\operatorname{reach}_{F}(B)$ is non-negative.

(c) The interval $I=[\ell(B)+1, s-1]$ is A-heavy.

(d) length $(t)=\#_{\mathrm{h}}(I)+\#_{\mathrm{H}}(I)+\operatorname{length}(B)$.

Fact 4. $(a) \Longrightarrow(b) \Longrightarrow(c)$ In addition, if we assume $(d)$ then $(c) \Longrightarrow(b) \Longrightarrow(a)$

Fact 4 can be seen as a refinement of Fact 1 when $F$ is a closed fork.

Proof.

(a) implies (b) An adversarial extension of $B$ contains only adversarial vertices from $I$. If this extension is viable at the onset of slot $s, \#_{\mathrm{A}}(I)$ must be at least $\operatorname{gap}_{F}(B)$. Since $\operatorname{reserve}_{F}(B)=\#_{\mathrm{A}}(I)$, we have $\operatorname{reach}_{F}(B)=$ $\operatorname{reserve}_{F}(B)-\operatorname{gap}_{F}(B) \geq 0$.

(b) implies (c) By assumption, $\operatorname{reach}_{F}(B)=\operatorname{reserve}_{F}(B)-\operatorname{gap}_{F}(B) \geq 0 . t$ contains at least $\#_{\mathrm{h}}(I)+\#_{\mathrm{H}}(I)$ vertices from the interval $I$; hence, $\operatorname{gap}_{F}(B) \geq \#_{\mathrm{h}}(I)+\#_{\mathrm{H}}(I)$. Since $\operatorname{reserve}_{F}(B)=\#_{\mathrm{A}}(I)$, it follows that $\#_{\mathrm{A}}(I) \geq \#_{\mathrm{h}}(I)+\#_{\mathrm{H}}(I)$.

(d) and (c) implies (b) Since $I$ is A-heavy, $\operatorname{reserve}_{F}(B)=\#_{\mathrm{A}}(I) \geq \#_{\mathrm{h}}(I)+\#_{\mathrm{H}}(I)$. However, since (d) holds, the latter quantity equals length $(t)-\operatorname{length}(B)=\operatorname{gap}_{F}(B)$. It follows that $\operatorname{reach}_{F}(B)=\operatorname{reserve}_{F}(B)-$ $\operatorname{gap}_{F}(B) \geq 0$.

(d) and (b) implies (a) $I$ contains at least $\operatorname{gap}_{F}(B)$ adversarial slots. We can use these slots augment $B$ into an adversarial tine $t^{\prime}$ of length at least length $(t)$. Thus $t^{\prime}$ will be viable at the onset of slot $s$.

Observe that for any characteristic string $x$, one can extend (i.e., augment) a closed fork prefix $F \vdash x$ into a larger closed fork $F^{\prime} \vdash x 0$ so that $F \sqsubseteq F^{\prime}$. A conservative extension is a minimal extension in that it consumes the least amount of reserve (cf. Definition 13), leaving the remaining reserve to be used in future. Extensions and, in particular, conservative extensions play a critical role in the exposition that follows.

Definition 15 (Extensions). Let $w \in\{\mathrm{h}, \mathrm{H}, \mathrm{A}\}^{*}$ be a characteristic string and $F$ a closed fork for $w$. Let $F^{\prime}$ be a closed fork for $w b, b \in\{\mathrm{h}, \mathrm{H}\}$ so that $F \sqsubseteq F^{\prime}$. We say that $F^{\prime}$ is an extension of $F$ if every honest vertex in $F^{\prime}$ either belongs to $F$ or has label $|w|+1$. Let $\sigma \in F^{\prime}$ be an honest vertex with $\ell(\sigma)=|w|+1$ and let $s$ be the longest honest prefix of $\sigma$. (Necessarily, $s \in F$.) We say that $\sigma$ is an extension of $s$. The new tine $\sigma$ is a conservative extension if $\operatorname{height}\left(F^{\prime}\right)=\operatorname{height}(F)+1$.

Since $F^{\prime}$ is closed, all longest tines in $F^{\prime}$ are honest and they have label $|w|+1$. Let $\hat{t}$ be the unique longest honest tine in $F^{\prime}$ under the consistent longest-chain selection rule in Axiom $\mathbf{A 0}^{\prime}$ Now consider a tine $\sigma \in S$. Since $\sigma$ is honest, it follows that length $(\sigma) \geq 1+\operatorname{height}(F)=1+\operatorname{length}(s)+\operatorname{gap}_{F}(s)$ where $s \in F$ is the longest honst prefix of $\sigma$. The root-to-leaf path in $F^{\prime}$ that ends at $\sigma$ contains at least $\operatorname{gap}_{F}(s)$ adversarial vertices $u \in F^{\prime}$ so that

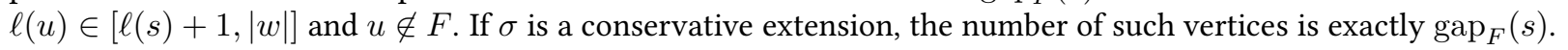

Fact 5 (Extensions and reach). Let $b \in\{\mathrm{h}, \mathrm{H}\}$. Let $F \vdash w$ and $F^{\prime} \vdash w b$ be closed forks so that $F \sqsubseteq F^{\prime}$ and $F^{\prime}$ is obtained from $F$ via one or more extensions $\sigma \in F^{\prime}, \ell(\sigma)=|w|+1$. Then $\operatorname{reach}_{F^{\prime}}(t) \leq \operatorname{reach}_{F}(t)-1$ for everyt $t \in F$. If all these extensions are conservative, then $\operatorname{reach}_{F^{\prime}}(t)=\operatorname{reach}_{F}(t)-1$ for every $t \in F$. Furthermore, a conservative extension $\sigma$ satisfies reach $_{F^{\prime}}(\sigma)=0$.

The above fact follows from the claims below. 
Claim 1. Let $b \in\{\mathrm{h}, \mathrm{H}\}$. Consider a closed fork $F \vdash w$ and some closed fork $F^{\prime} \vdash w b$ such that $F \sqsubseteq F^{\prime}$. Ift $\in F$ then $\operatorname{reach}_{F^{\prime}}(t) \leq \operatorname{reach}_{F}(t)-1$. The inequality becomes and equality if $F^{\prime}$ is obtained via conservative extensions from $F$.

Proof. We know that reach $F_{F^{\prime}}(t)=$ reserve $_{F^{\prime}}(t)-\operatorname{gap}_{F^{\prime}}(t)$. From $F$ to $F^{\prime}$, the length of the longest tine increases by at least one, and the length of $t$ does not change. It follows that $\operatorname{gap}_{F^{\prime}}(t) \geq \operatorname{gap}_{F}(t)+1$. The inequality becomes an equality if $F^{\prime}$ is obtained from $F$ via only conservative extensions. The reserve of $t$ does not change, because there are no new As in the characteristic string. Therefore, $\operatorname{reach}_{F^{\prime}}(t)=\operatorname{reserve}_{F^{\prime}}(t)-\operatorname{gap}_{F^{\prime}}(t) \leq \operatorname{reserve}_{F}(t)-$ $\operatorname{gap}_{F}(t)-1=\operatorname{reach}_{F}(t)-1$.

Claim 2. Conservative extensions have reach zero.

Proof. Let $b \in\{\mathrm{h}, \mathrm{H}\}$. Consider closed forks $F \vdash w, F^{\prime} \vdash w b$ such that $F \sqsubseteq F^{\prime}$. Let $t \in F^{\prime}$ be a conservative extension. This means $t$ is honest, $\ell(t)=|w|+1$, and $t$ is a longest tine in $F^{\prime}$. The last statement implies $\operatorname{gap}_{F^{\prime}}(t)=0$. Since reserve $_{F^{\prime}}(t)=0$, it follows that $\operatorname{reach}_{F^{\prime}}(t)=\operatorname{reserve}_{F^{\prime}}(t)-\operatorname{gap}_{F^{\prime}}(t)=0$.

\subsection{Relative margin}

Definition 16 (The $\sim_{x}$ relations). For two tines $t_{1}$ and $t_{2}$ of a fork $F$, we write $t_{1} \sim t_{2}$ when $t_{1}$ and $t_{2}$ share an edge; otherwise we write $t_{1} \nsim t_{2}$. We generalize this equivalence relation to reflect whether tines share an edge over a particular suffix of $w$ : for $w=x y$ we define $t_{1} \sim_{x} t_{2}$ if $t_{1}$ and $t_{2}$ share an edge that terminates at some node labeled with an index in $y$; otherwise, we write $t_{1} \nsim_{x} t_{2}$ (observe that in this case the paths share no vertex labeled by a slot associated with $y$ ). We sometimes call such pairs of tines disjoint (or, if $t_{1} \nsim_{x} t_{2}$ for a string $w=x y$, disjoint over $y$ ). Note that $\sim$ and $\sim_{\varepsilon}$ are the same relation.

Definition 17 (Margin). The margin of a fork $F \vdash w$, denoted $\mu(F)$, is defined as

$$
\mu(F)=\max _{t_{1} \nsim t_{2}}\left(\min \left\{\operatorname{reach}\left(t_{1}\right), \operatorname{reach}\left(t_{2}\right)\right\}\right)
$$

where this maximum is extended over all pairs of disjoint tines of F; thus margin reflects the "second best" reach obtained over all disjoint tines. In order to study splits in the chain over particular portions of a string, we generalize this to define a "relative" notion of margin: If $w=x y$ for two strings $x$ and $y$ and, as above, $F \vdash w$, we define

$$
\mu_{x}(F)=\max _{t_{1} \nsim_{x} t_{2}}\left(\min \left\{\operatorname{reach}\left(t_{1}\right), \operatorname{reach}\left(t_{2}\right)\right\}\right)
$$

Note that $\mu_{\varepsilon}(F)=\mu(F)$.

For convenience, we once again overload this notation to denote the margin of a string. $\mu(w)$ refers to the maximum value of $\mu(F)$ over all possible closed forks $F$ for a characteristic string $w$ :

$$
\mu(w)=\max _{\substack{F \vdash w, F \text { closed }}} \mu(F) .
$$

Likewise, if $w=x y$ for two strings $x$ and $y$ we define

$$
\mu_{x}(y)=\max _{\substack{F \vdash w \\ F \text { closed }}} \mu_{x}(F) .
$$

Note that, at least informally, disjoint tines with large reach are of natural interest to an adversary who wants to build an $x$-balanced fork, since such a fork contains two (partially disjoint) long tines. It is easy to see that if $w=x x^{\prime} y$ and $\mu_{x x^{\prime}}(y)$ is negative then $\mu_{x}\left(x^{\prime} y\right)$ is negative as well.

The theorem below shows how to recursively compute $\mu_{x}(y)$ for a given decomposition $w=x y$.

Theorem 5. Let $\varepsilon$ be the empty string and $b \in\{\mathrm{h}, \mathrm{H}\}$. Then $\rho(\varepsilon)=0$ and, for all nonempty strings $w \in\{\mathrm{h}, \mathrm{H}, \mathrm{A}\}^{*}$

$$
\rho(w \mathrm{~A})=\rho(w)+1, \quad \text { and } \quad \rho(w b)= \begin{cases}0 & \text { if } \rho(w)=0 \\ \rho(w)-1 & \text { otherwise }\end{cases}
$$


Furthermore, for any strings $x, y \in\{\mathrm{h}, \mathrm{H}, \mathrm{A}\}^{*}, \mu_{x}(\varepsilon)=\rho(x)$,

$$
\mu_{x}(y \mathrm{~A})=\mu_{x}(y)+1, \quad \text { and } \quad \mu_{x}(y b)= \begin{cases}0 & \text { if } \rho(x y)>\mu_{x}(y)=0 \\ 0 & \text { if } \rho(x y)=\mu_{x}(y)=0 \text { and } b=\mathrm{H} \\ \mu_{x}(y)-1 & \text { otherwise. }\end{cases}
$$

The proof of Theorem[5is given in Section7 Let $w$ be a characteristic string and let $m, k \in \mathbb{N}$ so that $m+k \leq|w|$. Let $x \prec w,|x|=m-1$ and $x y \preceq w,|x y| \geq m+k$. If the symbols in $w$ are independent and identically distributed, the recursive formulation in (14) implies an algorithm - which takes time and space $O\left(|w|^{3}\right)$ - for computing the probability that $\mu_{x}(y) \geq 0$. But this is exactly the probability that slot $m$ is not $k$-settled, according to (1) and Lemma 1 below. In Section 6.6 we describe this algorithm in more detail and compile some explicit values for this probability.

\subsection{Balanced forks, settlement violations, and relative margin}

A natural structure we can use to reason about settlement times (see Definition 3) is that of a "balanced fork."

Definition 18 (Balanced fork). A fork $F$ is balanced if it contains a pair of tines $t_{1}$ and $t_{2}$ for which $t_{1} \nsim t_{2}$ and length $\left(t_{1}\right)=\operatorname{length}\left(t_{2}\right)=$ height $(F)$. We define a relative notion of balance as follows: a fork $F \vdash x y$ is $x$-balanced if it contains a pair of tines $t_{1}$ and $t_{2}$ for which $t_{1} \chi_{x} t_{2}$ and length $\left(t_{1}\right)=\operatorname{length}\left(t_{2}\right)=\operatorname{height}(F)$.

Thus, balanced forks contain two completely disjoint, maximum-length tines, while $x$-balanced forks contain two maximum-length tines that may share edges in $x$ but must be disjoint over the rest of the string. See Figures 2 and 3 for examples of balanced forks.

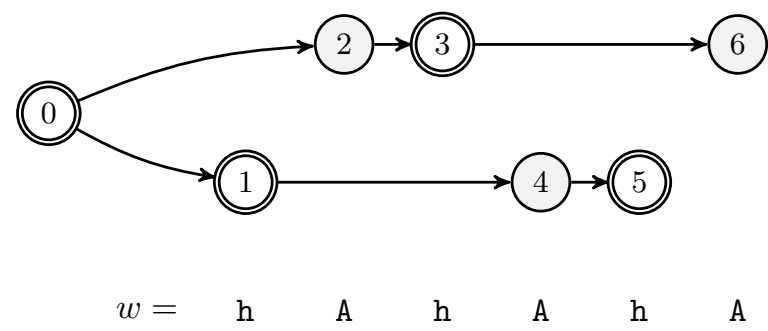

Figure 2: A balanced fork

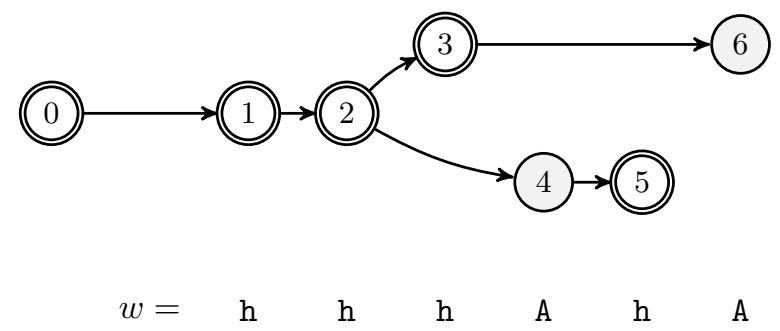

Figure 3: An $x$-balanced fork, where $x=\mathrm{hh}$

A fundamental question arising in typical blockchain settings is how to determine settlement time, the delay after which the contents of a particular block of a blockchain can be considered stable. The existence of a balanced fork is a precise indicator for "settlement violations" in this sense. Specifically, consider a characteristic string $x y$ and a transaction appearing in a block associated with the first slot of $y$ (that is, slot $|x|+1$ ). One clear violation of settlement at this point of the execution is the existence of two chains-each of maximum length-which diverge prior to $y$; in particular, this indicates that there is an $x$-balanced fork $F$ for $x y$. Let us record this observation below 3

Observation 2. Let $s, k \in \mathbb{N}$ be given and let $w$ be a characteristic string. Slot $s$ is not $k$-settled for the characteristic string $w$ if there exist a decomposition $w=x y z$, where $|x|=s-1$ and $|y| \geq k+1$, and an $x$-balanced fork for $x y$.

In particular, to provide a rigorous $k$-slot settlement guarantee-which is to say that the transaction can be considered settled once $k$ slots have gone by-it suffices to show that with overwhelming probability in choice of the characteristic string determined by the leader election process (of a full execution of the protocol), no such forks are possible. Specifically, if the protocol runs for a total of $T$ time steps yielding the characteristics string $w=x y$ (where $w \in\{0,1\}^{T}$ and the transaction of interest appears in slot $|x|+1$ as above) then it suffices to ensure that

\footnotetext{
${ }^{3}$ A balanced fork in [3] had the property that at least one maximum-length tine was adversarial. But this is not true in our setting since we allow multiply honest slots.
} 
there is no $x$-balanced fork for $x \hat{y}$, where $\hat{y}$ is an arbitrary prefix of $y$ of length at least $k+1$. Note that for systems adopting the longest chain rule, this condition must necessarily involve the entire future dynamics of the blockchain. We remark that our analysis below will in fact let us take $T=\infty$.

Let $w$ be a characteristic string. Writing $w=x y$, consider any tine-pair $\left(t_{x}, t_{\rho}\right)$ in a fork $F \vdash w$ so that $\operatorname{reach}_{F}\left(t_{\rho}\right)=\rho(F)$ and $t_{x}$ is $y$-disjoint with $t_{\rho}$. Observe that if $\mu_{x}(y)<0$ then $\operatorname{reach}_{F}\left(t_{x}\right)<0$.

Fact 6. Let $x y \in\{\mathrm{h}, \mathrm{H}, \mathrm{A}\}^{*}$ be a characteristic string. There is no $x$-balanced fork for $x y$ if and only if $\mu_{x}(y)<0$.

Proof sketch. If a fork $F \vdash x y$ satisfies $\mu_{x}(F) \geq 0$, it contains two $y$-disjoint tines $t_{1}, t_{2}$, each with a non-negative reach, so that $\min \left(\operatorname{reach}\left(t_{1}\right), \operatorname{reach}\left(t_{2}\right)\right)=\mu_{x}(F)$. As reserve $\left(t_{i}\right) \geq \operatorname{gap}\left(t_{i}\right)$ for $i \in\{1,2\}$, we can extend these tines using only new adversarial vertices so that both these extensions have the maximum length in the augmented fork. Thus the augmented fork is $x$-balanced.

On the other hand, if a fork $F \vdash x y$ is $x$-balanced, there must be two $y$-disjoint maximum-length tines $t_{1}, t_{2} \in F$. As the gap of a maximum-length tine is zero, we must have reach $\left(t_{i}\right)=\operatorname{reserve}\left(t_{i}\right) \geq 0$ for $i \in\{1,2\}$. It follows that $\mu_{x}(y) \geq \mu_{x}(F) \geq \min _{i}$ reach $\left(t_{i}\right) \geq 0$.

\subsection{Relative margin to characterize the UVP}

Let $w$ be a characteristic string. Recall that in Theorem 3 we showed that whether a slot has the UVP in $w-$ a structural property of the forks for $w$ - is characterized by the "Catalan-ness" of the said slot. Below, we show that relative margin has the same expressive power as the Catalan slots in terms of characterizing the UVP.

Lemma 1. Let $T \in \mathbb{N}, w \in\{\mathrm{h}, \mathrm{H}, \mathrm{A}\}^{T}$, and $s \in[T]$ so that $w_{s}=\mathrm{h}$. Let $x=w_{1} \ldots w_{s-1}$. Slot $s$ has the UVP in $w$ if and only if for every prefix $x y \preceq w, \mu_{x}(y)<0$.

Proof.

The $\Longleftarrow$ direction. Suppose that for every prefix $x y \preceq w$ where $|y| \geq 1$, we have $\mu_{x}(y)<0$. We wish to show that $s$ has the UVP in $w$.

Let $F$ be any fork for $x y$ and let $t \in F, \ell(t) \leq s-1$ be an honest tine. Since it is disjoint with any tine in $F$ over the suffix $y, \operatorname{reach}(t)<0$ and, by Fact 4 does not have an adversarial extension $t^{\prime} \in F, t \prec t^{\prime}$ that is viable at the onset of slot $|x y|+1$. Therefore, if a tine in $F$ is viable at the onset of slot $|x y|+1$, it must contain an honest vertex with label at least $s$. However, since an honest vertex builds only on top of a viable tine, it follows that any viable tine must contain the unique honest vertex with label $s$.

The $\Longrightarrow$ direction. Suppose $s$ has the UVP in $w$. Let $k \in[s, T]$ be an integer and write $w=x y z$ with $|x y|=k$. (Note that $y_{1}=w_{s}$.) We wish to show that $\mu_{x}(y)<0$.

Let $F$ be any fork for $x y$. Since slot $s$ belongs to $y, F$ cannot contain two tines such that (i) both tines are viable at the onset of slot $|x y|+1$ and, at the same time, (ii) disjoint over the length of $y$ since they must contain the unique vertex with label $s$. In particular, $F$ cannot be $x$-balanced. As $F$ was an arbitrary fork for $x y$, no fork for $x y$ can be $x$-balanced for our choice of $k$. We use Fact 6 to conclude that $\mu_{x}(y)$ must be negative.

\subsection{An optimal online adversary against slot settlement}

Let $w$ be a characteristic string. For a fixed decomposition $w=x y$, there is an adversary $y^{4}$ who builds a fork $F \vdash x y$ so that the $\mu_{x}(F)$ is at least as large as the right-hand side of (14). However, in light of Lemma1 if an adversary wants to violate the settlement of all possible slots of $w$ at once, he needs to produce a fork $F$ for $w$ so that $\mu_{x}(F) \geq 0$

\footnotetext{
${ }^{4}$ Specifically, let $w^{\prime}=x y b$ where $b \in\{\mathrm{h}, \mathrm{H}, \mathrm{A}\}$. This strategy recursively builds a closed fork $F \vdash x y$. Then, upon encountering $b$, it augments $F$ by making zero, one, or two conservative extensions, as follows: If $b=\mathrm{A}$, it does nothing. If $b=\mathrm{h}$, it extends a zero-reach tine if possible; otherwise, it extends a maximum-reach tine. If $b=\mathrm{H}$, it extends a pair of tines that witness $\mu_{x}(F)$. By following the arguments in [4], one can show that if $\mu_{x}(F)=\mu_{x}(y)$ then $\mu_{x}\left(F^{\prime}\right)$ is at least as large as the right-hand side in [14.
} 
for every prefix $x \preceq w$. In Figure 4 we describe a strategy $\mathcal{A}^{*}$ which does even better: it produces a fork $F$ so that $\mu_{x}(F)=\mu_{x}(y)$ for every prefix $x \preceq w$.

$\mathcal{A}^{*}$ builds a fork for $w=w_{1} \ldots w_{n+1}$ in an online fashion, i.e., it scans $w$ once, from left to right, maintains a fork $F_{n}$ after scanning the first $n$ symbols, and augments $F_{n}$ by conservatively extending zero-reach tine(s) using label $n+1$. Specifically, if $w_{n+1}=\mathrm{A}, \mathcal{A}^{*}$ does nothing. If $w_{n+1}=\mathrm{h}$, it (obviously) makes a single extension. Now suppose $w_{n+1}=\mathrm{H}$. It still makes a single extension if either $F_{n}$ contains exactly one zero-reach tine or $F_{n}$ 's reach is positive. Otherwise, if $\rho\left(F_{n}\right)=0$ and there are at least two zero-reach tines in $F_{n}, \mathcal{A}^{*}$ extends two zero-reach tines that diverge earliest in $F_{n}$.

\section{The strategy $\mathcal{A}^{*}$}

Let $n$ be a non-negative integer, $w \in\{\mathrm{h}, \mathrm{H}, \mathrm{A}\}^{n}$, and $w_{n+1} \in\{\mathrm{h}, \mathrm{H}, \mathrm{A}\}$. If $n=0$, set $F_{0} \vdash \varepsilon$ as the trivial fork comprising a single vertex. Otherwise, let $F_{n}$ be the closed fork built recursively by $\mathcal{A}^{*}$ for the string $w$. If $w_{n+1}=\mathrm{A}$, output $F_{n}$ (as a fork for $w w_{n+1}$ ). Otherwise, let $Z$ and $R$ be the set of zero-reach tines and maximum-reach tines in $F_{n}$, respectively.

1. Identify a set $S$ as follows: If $|Z|=1$ then set $S=Z$. Otherwise, let $r_{1} \in R, z_{1} \in Z$ be two tines so that $\ell\left(r_{1} \cap z_{1}\right)=\min \{\ell(r \cap z): r \in R, z \in Z\}$ and set

$$
S= \begin{cases}\left\{z_{1}\right\} & \text { if } w_{n+1}=\mathrm{h} \text { or } \rho\left(F_{n}\right) \geq 1, \\ \left\{z_{1}, r_{1}\right\} & \text { otherwise }\end{cases}
$$

2. Conservatively extend each tine in $S$ using label $n+1$. Let $F_{n+1} \vdash w w_{n+1}$ be the new closed fork. Output $F_{n+1}$.

Figure 4: Optimal online adversary $\mathcal{A}^{*}$

Definition 19 (Canonical fork). A canonical fork for $w \in\{\mathrm{h}, \mathrm{H}, \mathrm{A}\}^{*}$ is a closed fork $F \vdash w$ so that $\rho(F)=\rho(w)$ and, for all prefixes $x \prec w, \mu_{x}(F)=\mu_{x}(y)$. If $|w|=0, F$ is the unique fork with a single (honest) vertex and no edge.

It is not obvious whether a canonical fork always exists or whether it can be found algorithmically. The theorem below gives us the assurance:

Theorem 6. Let $w \in\{\mathrm{h}, \mathrm{H}, \mathrm{A}\}^{*}$. The strategy $\mathcal{A}^{*}$ in Figure 4 outputs a canonical fork for $w$.

That is, for every characteristic string $w$ there is a fork $F \vdash w$ so that for every prefix $x \preceq w, \mu_{x}(F)=\mu_{x}(y)$. Note that if one's objective is to create a fork which contains many early-diverging tine-pairs (that witness large relative margins), a canonical fork is the best one can hope for. This is why $\mathcal{A}^{*}$ is called an optimal online adversary. The proof of Theorem 6 is given in Section 7

\subsection{An algorithm to compute exact settlement probabilities}

Let $m, k \in \mathbb{N}, \epsilon \in(0,1]$, and $p_{\mathrm{h}} \in(0,(1+\epsilon) / 2]$. Let $T=m+k, \alpha=(1-\epsilon) / 2$, and $p_{\mathrm{H}}=1-\alpha-p_{\mathrm{h}}$. Let $w \in\{\mathrm{h}, \mathrm{H}, \mathrm{A}\}^{T}$ such that the symbols $w_{i}, i \in[T]$ are i.i.d. with $\operatorname{Pr}\left[w_{i}=\mathrm{A}\right]=\alpha, \operatorname{Pr}\left[w_{i}=\mathrm{h}\right]=p_{\mathrm{h}}$, and $\operatorname{Pr}\left[w_{i}=\mathrm{H}\right]=p_{\mathrm{H}}$. Write $w$ as $w=x y$ where $|x|=m,|y|=k$. The recursive definition of relative margin (cf. Theorem 5 ) implies an algorithm for computing the probability $\operatorname{Pr}\left[\mu_{x}(y) \geq 0\right]$ in $O\left(T^{3}\right)$ time and space.

In typical circumstances, however, it is more interesting to establish an explicit upper bound on $\operatorname{Pr}\left[\mu_{x}(y) \geq\right.$ $0]$ where $|x| \rightarrow \infty$; this corresponds to the case where the distribution of the initial reach $\rho(x)$ is the dominant distribution $\mathcal{X}_{\infty}$ in (9). Due to dominance, $\mathcal{X}_{\infty}(m)$ serves as an upper bound on $\rho(x)$ for any finite $m=|x|$. For this purpose, one can implicitly maintain a sequence of matrices $M_{t}, t=0,1,2, \ldots, k$ such that $M_{0}(r, r)=\mathcal{X}_{\infty}(r)$ for all $0 \leq r \leq 2 k$ and the invariant

$$
M_{t}(r, s)=\operatorname{Pr}_{y:|y|=t}\left[\rho(x y)=r \text { and } \mu_{x}(y)=s\right]
$$


Table 1: Exact probabilities of $k$-settlement violations where the symbols $\mathrm{h}, \mathrm{H}, \mathrm{A}$ are independent and identically distributed as $\operatorname{Pr}[\mathrm{A}]=\alpha \in(0,0.5)$ and $\operatorname{Pr}[\mathrm{H}]=1-\alpha-\operatorname{Pr}[\mathrm{h}]$.

\begin{tabular}{|c|c|c|c|c|c|c|c|}
\hline \multirow{2}{*}{$\frac{\operatorname{Pr}[\mathrm{h}]}{1-\alpha}$} & \multirow{2}{*}{$k$} & \multicolumn{6}{|c|}{$\alpha$} \\
\hline & & 0.01 & 0.10 & 0.20 & 0.30 & 0.40 & 0.49 \\
\hline \multirow{5}{*}{1.0} & 100 & $5.70 \mathrm{E}-054$ & $5.10 \mathrm{E}-018$ & $2.28 \mathrm{E}-008$ & $8.00 \mathrm{E}-004$ & $1.37 \mathrm{E}-001$ & $9.05 \mathrm{E}-001$ \\
\hline & 200 & $1.64 \mathrm{E}-106$ & $9.82 \mathrm{E}-035$ & $1.61 \mathrm{E}-015$ & $1.60 \mathrm{E}-006$ & $3.36 \mathrm{E}-002$ & $8.73 \mathrm{E}-001$ \\
\hline & 300 & $4.70 \mathrm{E}-159$ & $1.89 \mathrm{E}-051$ & $1.14 \mathrm{E}-022$ & $3.25 \mathrm{E}-009$ & $8.52 \mathrm{E}-003$ & $8.50 \mathrm{E}-001$ \\
\hline & 400 & $1.35 \mathrm{E}-211$ & $3.64 \mathrm{E}-068$ & $8.02 \mathrm{E}-030$ & $6.59 \mathrm{E}-012$ & $2.18 \mathrm{E}-003$ & $8.29 \mathrm{E}-001$ \\
\hline & 500 & $1.02 \mathrm{E}-264$ & $3.90 \mathrm{E}-085$ & $4.00 \mathrm{E}-037$ & $1.10 \mathrm{E}-014$ & $5.16 \mathrm{E}-004$ & $8.05 \mathrm{E}-001$ \\
\hline \multirow{5}{*}{0.9} & 100 & $9.75 \mathrm{E}-052$ & $1.24 \mathrm{E}-017$ & $3.24 \mathrm{E}-008$ & $9.27 \mathrm{E}-004$ & $1.44 \mathrm{E}-001$ & $9.08 \mathrm{E}-001$ \\
\hline & 200 & $3.04 \mathrm{E}-102$ & $4.95 \mathrm{E}-034$ & $2.96 \mathrm{E}-015$ & $2.03 \mathrm{E}-006$ & $3.60 \mathrm{E}-002$ & $8.77 \mathrm{E}-001$ \\
\hline & 300 & $9.46 \mathrm{E}-153$ & $1.98 \mathrm{E}-050$ & $2.71 \mathrm{E}-022$ & $4.50 \mathrm{E}-009$ & $9.30 \mathrm{E}-003$ & $8.53 \mathrm{E}-001$ \\
\hline & $\overline{400}$ & $2.95 \mathrm{E}-203$ & $7.91 \mathrm{E}-067$ & $2.48 \mathrm{E}-029$ & $9.96 \mathrm{E}-012$ & $2.43 \mathrm{E}-003$ & $8.33 \mathrm{E}-001$ \\
\hline & $\overline{500}$ & $1.83 \mathrm{E}-254$ & $1.63 \mathrm{E}-083$ & $1.54 \mathrm{E}-036$ & $1.78 \mathrm{E}-014$ & $5.80 \mathrm{E}-004$ & $8.08 \mathrm{E}-001$ \\
\hline \multirow{5}{*}{0.8} & $\overline{100}$ & $6.16 \mathrm{E}-048$ & $4.13 \mathrm{E}-017$ & $5.10 \mathrm{E}-008$ & $1.11 \mathrm{E}-003$ & $1.53 \mathrm{E}-001$ & $9.11 \mathrm{E}-001$ \\
\hline & 200 & $7.58 \mathrm{E}-095$ & $4.61 \mathrm{E}-033$ & $6.58 \mathrm{E}-015$ & $2.73 \mathrm{E}-006$ & $3.91 \mathrm{E}-002$ & $8.81 \mathrm{E}-001$ \\
\hline & $\overline{300}$ & $9.32 \mathrm{E}-142$ & $5.14 \mathrm{E}-049$ & $8.48 \mathrm{E}-022$ & $6.78 \mathrm{E}-009$ & $1.04 \mathrm{E}-002$ & $8.57 \mathrm{E}-001$ \\
\hline & 400 & $1.15 \mathrm{E}-188$ & $5.74 \mathrm{E}-065$ & $1.09 \mathrm{E}-028$ & $1.68 \mathrm{E}-011$ & $2.77 \mathrm{E}-003$ & $8.38 \mathrm{E}-001$ \\
\hline & $\overline{500}$ & $1.94 \mathrm{E}-236$ & $3.02 \mathrm{E}-081$ & $9.16 \mathrm{E}-036$ & $3.28 \mathrm{E}-014$ & $6.70 \mathrm{E}-004$ & $8.12 \mathrm{E}-001$ \\
\hline \multirow{5}{*}{0.5} & 100 & $4.80 \mathrm{E}-028$ & $6.53 \mathrm{E}-014$ & $6.21 \mathrm{E}-007$ & $2.80 \mathrm{E}-003$ & $1.99 \mathrm{E}-001$ & $9.26 \mathrm{E}-001$ \\
\hline & 200 & $2.46 \mathrm{E}-055$ & $6.31 \mathrm{E}-027$ & $6.40 \mathrm{E}-013$ & $1.31 \mathrm{E}-005$ & $5.86 \mathrm{E}-002$ & $8.98 \mathrm{E}-001$ \\
\hline & 300 & $1.26 \mathrm{E}-082$ & $6.10 \mathrm{E}-040$ & $6.60 \mathrm{E}-019$ & $6.19 \mathrm{E}-008$ & $1.76 \mathrm{E}-002$ & $8.77 \mathrm{E}-001$ \\
\hline & $\overline{400}$ & $6.46 \mathrm{E}-110$ & $5.90 \mathrm{E}-053$ & $6.81 \mathrm{E}-025$ & $2.92 \mathrm{E}-010$ & $5.33 \mathrm{E}-003$ & $8.59 \mathrm{E}-001$ \\
\hline & 500 & $1.28 \mathrm{E}-138$ & $1.75 \mathrm{E}-066$ & $3.65 \mathrm{E}-031$ & $9.61 \mathrm{E}-013$ & $1.39 \mathrm{E}-003$ & $8.31 \mathrm{E}-001$ \\
\hline \multirow{5}{*}{0.25} & 100 & $1.22 \mathrm{E}-012$ & $3.13 \mathrm{E}-008$ & $8.94 \mathrm{E}-005$ & $1.65 \mathrm{E}-002$ & $3.17 \mathrm{E}-001$ & $9.48 \mathrm{E}-001$ \\
\hline & 200 & $1.51 \mathrm{E}-024$ & $1.06 \mathrm{E}-015$ & $9.36 \mathrm{E}-009$ & $3.36 \mathrm{E}-004$ & $1.25 \mathrm{E}-001$ & $9.27 \mathrm{E}-001$ \\
\hline & 300 & $1.86 \mathrm{E}-036$ & $3.62 \mathrm{E}-023$ & $9.80 \mathrm{E}-013$ & $6.86 \mathrm{E}-006$ & $4.94 \mathrm{E}-002$ & $9.10 \mathrm{E}-001$ \\
\hline & 400 & $2.30 \mathrm{E}-048$ & $1.23 \mathrm{E}-030$ & $1.03 \mathrm{E}-016$ & $1.40 \mathrm{E}-007$ & $1.96 \mathrm{E}-002$ & $8.96 \mathrm{E}-001$ \\
\hline & 500 & $5.06 \mathrm{E}-062$ & $7.72 \mathrm{E}-039$ & $4.06 \mathrm{E}-021$ & $1.66 \mathrm{E}-009$ & $6.20 \mathrm{E}-003$ & $8.65 \mathrm{E}-001$ \\
\hline \multirow{5}{*}{0.01} & 100 & $3.77 \mathrm{E}-001$ & $4.91 \mathrm{E}-001$ & $6.38 \mathrm{E}-001$ & $7.95 \mathrm{E}-001$ & $9.31 \mathrm{E}-001$ & $9.97 \mathrm{E}-001$ \\
\hline & 200 & $1.42 \mathrm{E}-001$ & $2.41 \mathrm{E}-001$ & $4.08 \mathrm{E}-001$ & $6.34 \mathrm{E}-001$ & $8.72 \mathrm{E}-001$ & $9.95 \mathrm{E}-001$ \\
\hline & 300 & $5.37 \mathrm{E}-002$ & $1.18 \mathrm{E}-001$ & $2.61 \mathrm{E}-001$ & $5.06 \mathrm{E}-001$ & $8.17 \mathrm{E}-001$ & $9.94 \mathrm{E}-001$ \\
\hline & 400 & $2.03 \mathrm{E}-002$ & $5.81 \mathrm{E}-002$ & $1.67 \mathrm{E}-001$ & $4.04 \mathrm{E}-001$ & $7.66 \mathrm{E}-001$ & $9.92 \mathrm{E}-001$ \\
\hline & $\overline{500}$ & $7.89 \mathrm{E}-005$ & $3.23 \mathrm{E}-003$ & $2.71 \mathrm{E}-002$ & $1.40 \mathrm{E}-001$ & $4.83 \mathrm{E}-001$ & $9.54 \mathrm{E}-001$ \\
\hline
\end{tabular}

is satisfied for every integer $t \in[1, k], r \in[0,2 k]$, and $s \in[-2 k, 2 k]$. Here, $M(i, j)$ denotes the entry at the $i$ th row and $j$ th column of a matrix $M$. Observe that $M_{t}(r, s)$ can be computed solely from the relevant neighboring cells of $M_{t-1}$, that is, from the values $M_{t-1}(r \pm 1, s \pm 1)$. Of course, only the transitions approved by (14) should be considered.

Finally, one can compute $\operatorname{Pr}\left[\mu_{x}(y) \geq 0\right]$ by summing $M_{k}(r, s)$ for $r, s \geq 0$. This is precisely the probability that, given a characteristic string $x y$ where $|x| \rightarrow \infty$, the slot $|x|+1$ incurs a $|y|$-settlement violation. Table 1)(on page 26) contains these probabilities for various values of $\alpha,|y|$, and $p_{\mathrm{h}}$.

A C++ implementation of the above algorithm is publicly available at https://github.com/saad0105050/multihonest-code [11].

\section{Proofs of Theorem 5 and Theorem 6}

The proof of Theorem 5 is presented in two parts. Let $w \in\{\mathrm{h}, \mathrm{H}, \mathrm{A}\}^{*}$. First, for a given decomposition $w=x y$, we prove an upper bound on $\mu_{x}(y)$. Next, considering the fork $F \vdash w$ built by the strategy Adversary* (see Figure 4 ), 
we show that for every decomposition $w=x y, \mu_{x}(F)$ is at least as large as the upper bound proven in the first part; thus $F$ is canonical.

As a warm-up, we start with the following claim.

Claim 3. $\rho(\varepsilon)=0$. For any $x, y \in\{\mathrm{h}, \mathrm{H}, \mathrm{A}\}^{*}, \mu_{x}(\varepsilon)=\rho(x), \rho(x y \mathrm{~A})=\rho(x y)+1$, and $\mu_{x}(y \mathrm{~A})=\mu_{x}(y)+1$.

Proof. The only possible fork for the empty string $\varepsilon$ contains a single honest vertex with reserve and gap both zero; hence $\rho(\varepsilon)=0$.

Let $F$ be a closed fork for the characteristic string $x y$. Let $t_{\rho}, t_{x} \in F$ be the two tines that witness $\mu_{x}(F)$, i.e., $\operatorname{reach}\left(t_{\rho}\right)=\rho(F), \operatorname{reach}_{F}\left(t_{x}\right)=\mu_{x}(F)$, and $t_{\rho}, t_{x}$ are disjoint over $y$.

In the base case, where $y=\varepsilon$, observe that any two tines of $F$ are disjoint over $y$. Moreover, a single tine $t \in F$ is disjoint with itself over the empty suffix $\varepsilon$. Therefore, the relative margin $\mu_{x}(\varepsilon)$ must be at least $\rho(x)$. As $\mu_{x}(F)$ can be no more than $\rho(x)$, it follows that $\mu_{x}(\varepsilon)=\rho(x)$.

Now consider a pair of closed forks $F \vdash x y$ and $F^{\prime} \vdash x y$ A such that $F \sqsubseteq F^{\prime}$ and $x, y \in\{\mathrm{h}, \mathrm{H}, \mathrm{A}\}^{*}$. We must have $F^{\prime}=F$ since $F^{\prime}$ is closed. In addition, for any tine $t \in F, \operatorname{reach}_{F^{\prime}}(t)=\operatorname{reach}_{F}(t)+1$ since the reserve has increased by one but the gap is unchanged (as no new tine is added). Therefore, $\rho(x y \mathrm{~A})=\rho(x y)+1$ and $\mu_{x}(y \mathrm{~A})=\mu_{x}(y)+1$.

\subsection{An upper bound on relative margin}

Proposition 1. Let $w, x, y \in\{\mathrm{h}, \mathrm{H}, \mathrm{A}\}^{*}$ and $b \in\{\mathrm{h}, \mathrm{H}\}$, Then

$$
\rho(x y b) \leq \begin{cases}0 & \text { if } \rho(x y)=0 \\ \rho(x y)-1 & \text { otherwise. }\end{cases}
$$

Furthermore,

$$
\mu_{x}(y b) \leq \begin{cases}0 & \text { if } \rho(x y)>\mu_{x}(y)=0 \\ 0 & \text { if } \rho(x y)=\mu_{x}(y)=0 \text { and } b=\mathrm{H} \\ \mu_{x}(y)-1 & \text { otherwise. }\end{cases}
$$

Proof. Suppose $F^{\prime} \vdash x y b$ is a closed fork such that $\rho(x y b)=\rho\left(F^{\prime}\right)$ and $\mu_{x}(y b)=\mu_{x}\left(F^{\prime}\right)$. Let $t_{\rho}, t_{x} \in F^{\prime}$ be a pair of $y$-disjoint tines such that $\operatorname{reach}_{F^{\prime}}\left(t_{\rho}\right)=\rho\left(F^{\prime}\right)$ and $\operatorname{reach}_{F^{\prime}}\left(t_{x}\right)=\mu_{x}\left(F^{\prime}\right)$. (If there are multiple candidates for $t_{\rho}$ or $t_{x}$, select the one with the smallest $\leq_{\pi}$ rank.) Let $F \vdash x y$ be the unique closed fork such that $F \sqsubseteq F^{\prime}$. Note that while $F^{\prime}$ is obtained from one or more extensions of $F$-tines, these extensions are not necessarily conservative. Recall that $\operatorname{reach}_{F^{\prime}}(t) \leq 0$ for any tine $t \in F^{\prime}, \ell(t)=|x y|+1$.

Proving (15). Let $A$ be the set of all $F^{\prime}$-tines with label $|x y|+1$. Let $\sigma \in A$ be the first tine in the $\leq_{\pi}$ ordering so that $\operatorname{reach}(\sigma)=\max _{t \in A}\left\{\operatorname{reach}_{F^{\prime}}(t)\right\}$. By Fact $5 \operatorname{reach}_{F^{\prime}}(\sigma) \leq 0$ and, in addition, for any $t \in F$, reach $F^{\prime}(t) \leq$ $\operatorname{reach}_{F}(t)-1$. Let $\hat{t}$ be the maximum-reach tine in $F$ with the smallest $\leq_{\pi}$ rank.

If $\rho(F)=0$ then $\operatorname{reach}_{F^{\prime}}(t)<0$ for all $t \in F$. Hence $t_{\rho}=\sigma$ and, consequently, $\rho(x y b) \leq 0$. If $\rho(F) \geq 2$ then $t_{\rho} \in F$ and, therefore, $\rho(x y b)=\operatorname{reach}_{F^{\prime}}\left(t_{\rho}\right) \leq \rho(F)-1 \leq \rho(x y)-1$. If $\rho(F)=1$ and $t_{\rho} \in F$ then, as before, $\rho(x y b)=\operatorname{reach}_{F^{\prime}}(\hat{t})=\operatorname{reach}_{F}(\hat{t})-1=\rho(F)-1 \leq \rho(x y)-1$. If $\rho(F)=1$ and $t_{\rho} \notin F$ then, as we have seen before, $\rho(x y b)=\operatorname{reach}_{F^{\prime}}(\sigma) \leq 0=\rho(F)-1 \leq \rho(x y)-1$. Thus we have proved (15).

Proving (16). If $\ell\left(t_{\rho}\right)=|x y|+1$ then we are done: by our preceding argument, $\operatorname{reach}_{F^{\prime}}\left(t_{\rho}\right) \leq 0$. On the other hand, Note that $t_{\rho} \notin F$ since, by Fact 5 reach of any $F$ tine can only decrease $t_{\rho}$ must have been an extension of a maximum-reach $F$-tine.

Case 1: $\rho(x y)>0$ and $\mu_{x}(y)=0$. We wish to show that $\mu_{x}(y b) \leq 0$. Suppose (toward a contradiction) that $\mu_{x}(y b)>0$. Then neither $t_{\rho}$ nor $t_{x}$ is a conservative extension because, as we proved in Claim 2 conservative extensions have reach zero. This means that $t_{\rho}$ and $t_{x}$ existed in $F$, and their $F$-reach was strictly greater than their $F^{\prime}$-reach (by Claim 1). Because $t_{\rho}$ and $t_{x}$ are disjoint over $y 0$, they must also be disjoint over $y$; therefore, 
$\mu_{x}(F)$ must be at least $\min \left(\operatorname{reach}_{F}\left(t_{\rho}\right), \operatorname{reach}_{F}\left(t_{x}\right)\right)$. It follows that $0=\mu_{x}(y) \geq \min \left(\operatorname{reach}_{F}\left(t_{\rho}\right), \operatorname{reach}_{F}\left(t_{x}\right)\right)>$ $\min \left(\operatorname{reach}_{F^{\prime}}\left(t_{\rho}\right), \operatorname{reach}_{F^{\prime}}\left(t_{x}\right)\right)=\mu_{x}\left(F^{\prime}\right)=\mu_{x}(y b)$. The last term is strictly positive by assumption and hence, a contradiction ensues.

Case 2: $\rho(x y)=0$. We wish to show that $(i) \mu_{x}(y b) \leq 0$ if $b=\mathrm{H}$ and $\mu_{x}(y)=0$, and $(i i) \mu_{x}(y b) \leq \mu_{x}(y)-1$ otherwise. First, we claim that $t_{\rho}$ must arise from an extension. Suppose, toward a contradiction, that $t_{\rho}$ is not an extension, i.e., $t_{\rho} \in F$. The fact that $t_{\rho}$ achieves the maximum reach in $F^{\prime}$ implies that $t_{\rho}$ has a non-negative reach since the longest honest tine always achieves reach zero. Furthermore, Claim 1]states that all $F$-tines see their reach decrease. Therefore, $t_{\rho} \in F$ must have had a strictly positive reach. But this contradicts the central assumption of the case, i.e., that $\rho(x y)=0$. Therefore, we conclude that $t_{\rho} \in F^{\prime} \backslash F$.

Let $s \in F$ be the tine-prefix of $t_{\rho} \in F^{\prime}$ so that $t_{\rho}$ is an extension of $s$. Observe that $\operatorname{reach}_{F}(s) \operatorname{must}_{\text {be }}$ non-negative since otherwise, $s$ could not have been extended. In fact, our assumption $\rho(x y)=0$ implies that $\operatorname{reach}_{F}(s)=0$. In addition, since $t_{x}$ and $t_{\rho}$ are disjoint over $y b$, so are $t_{x}$ and $s$.

If $b=\mathrm{h}, t_{\rho}$ is the only extension in $F^{\prime}$ and hence $t_{x}$ must be in $F$. Consequently, $\min \left(\operatorname{reach}_{F}(s), \operatorname{reach}_{F}\left(t_{x}\right)\right) \leq$ $\mu_{x}(y) \cdot \operatorname{Because}_{\operatorname{reach}_{F}}(s)=0$ and $\operatorname{reach}_{F}\left(t_{x}\right) \leq \rho(x y)=0$, it follows that $\operatorname{reach}_{F}\left(t_{x}\right) \leq \mu_{x}(y)$. Finally, since $t_{x} \in F$, Claim 1 tells us that reach $F^{\prime}\left(t_{x}\right)<\operatorname{reach}_{F}\left(t_{x}\right)$. Taken together, these two inequalities show that $\mu_{x}(y b)=\operatorname{reach}_{F^{\prime}}\left(t_{x}\right)<\operatorname{reach}_{F}\left(t_{x}\right) \leq \mu_{x}(y)$. The last inequality follows since $s$ and $t_{x}$ are disjoint over $y$ and $\operatorname{reach}_{F}(s)=0=\rho(x y)$. We conclude that $\mu_{x}(y b) \leq \mu_{x}(y)-1$.

If $b=\mathrm{H}$ and $\mu_{x}(y)<0$, we claim that $t_{x} \in F$. To see why, note that as $t_{x}$ is $y b$-disjoint with $t_{\rho}$, it must extend some $F$-tine $t$ that is $y$-disjoint with $t_{\rho}$. However, as $\mu_{x}(y)<0, t$ must have negative reach and hence cannot be extended into $t_{x}$; this is a contradiction. Therefore, $t_{x} \in F$ and we can apply the argument in the " $b=\mathrm{h}$ " case above to conclude that $\mu_{x}(y b) \leq \mu_{x}(y)-1$.

If $b=\mathrm{H}$ and $\mu_{x}(y)=0$, then there are two alternatives depending on whether $t_{x}$ is an extension. If $t_{x}$ is not an extension, we can apply the argument in the " $b=\mathrm{h}$ " case above and conclude that $\mu_{x}(y b) \leq \mu_{x}(y)-1=-1$. On the other hand, if $t_{x} \notin F$, both $t_{x}$ and $t_{\rho}$ are extensions and, by Fact 5 max $\left(\operatorname{reach}_{F^{\prime}}\left(t_{x}\right), \operatorname{reach}_{F^{\prime}}\left(t_{\rho}\right)\right) \leq 0$. In addition, Fact 5 states that for all $t \in F, \operatorname{reach}_{F^{\prime}}(t)<\operatorname{reach}_{F}(t) \leq \rho(x y)=0$. We conclude that $\mu_{x}(y b) \leq 0$

Case 3: $\rho(x y)>0$ and $\mu_{x}(y) \neq 0$. We wish to show that $\mu_{x}(y b) \leq \mu_{x}(y)-1$ or, equivalently, that $\mu_{x}(y b)<\mu_{x}(y)$. We will break this case into two sub-cases.

If both $t_{\rho}, t_{x} \in F$, then $\mu_{x}(y b)=\operatorname{reach}_{F^{\prime}}\left(t_{x}\right)<\operatorname{reach}_{F}\left(t_{x}\right) \leq \mu_{x}(y)$. Here, the first inequality follows from Fact 5 and the second inequality follows from the fact that $t_{x}, t_{\rho}$ is $y$-disjoint and $\operatorname{reach}\left(t_{x}\right)$ is at most $\operatorname{reach}\left(t_{\rho}\right)$ by design.

Otherwise, at least one of $t_{x}, t_{\rho}$ arose from an extension. Since reach $F_{F^{\prime}}\left(t_{x}\right) \leq \operatorname{reach}_{F^{\prime}}\left(t_{\rho}\right)$ by design, it follows that $\operatorname{reach}_{F^{\prime}}\left(t_{x}\right) \leq 0$ as the reach of an extension is at most zero. If $\mu_{x}(y)>0$ then we are done: $\mu_{x}(y b) \leq 0<$ $\mu_{x}(y)$. On the other hand, suppose $\mu_{x}(y)<0$. Recall the tine $s$ mentioned before. As $t_{x}$ is $y$-disjoint with $s$ and $\mu_{x}(y)$ is negative by assumption, $\operatorname{reach}_{F}\left(t_{x}\right)$ is at most $\mu_{x}(y)$. We conclude that $\mu_{x}(y b)=\operatorname{reach}_{F^{\prime}}\left(t_{x}\right)<$ $\operatorname{reach}_{F}\left(t_{x}\right) \leq \mu_{x}(y)$ where the inequality follows from Fact5.

\section{2 $\mathcal{A}^{*}$ simultaneously maximizes all relative margins}

Proposition 2. Let $w \in\{\mathrm{h}, \mathrm{H}, \mathrm{A}\}^{*}$ and $b \in\{\mathrm{h}, \mathrm{H}, \mathrm{A}\}$. Assume that Theorem 6 holds for characteristic strings of length $|w|$. Let $F^{\prime}$ be the fork built by $\mathcal{A}^{*}$ for the characteristic string $w b$. Then

$$
\rho\left(F^{\prime}\right) \geq \begin{cases}\rho(x y)+1 & \text { if } b=\mathrm{A}, \\ 0 & \text { if } b \in\{\mathrm{h}, \mathrm{H}\} \text { and } \rho(x y)=0 \\ \rho(x y)-1 & \text { otherwise }\end{cases}
$$


Furthermore, for any decomposition $w=x y,|y| \geq 0$,

$$
\mu_{x}\left(F^{\prime}\right) \geq \begin{cases}\mu_{x}(y)+1 & \text { ifb }=\mathrm{A} \\ 0 & \text { ifb } \in\{\mathrm{h}, \mathrm{H}\} \text { and } \rho(x y)>\mu_{x}(y)=0 \\ 0 & \text { ifb } b \mathrm{H} \text { and } \rho(x y)=\mu_{x}(y)=0 \\ \mu_{x}(y)-1 & \text { otherwise. }\end{cases}
$$

Proof. Let $w^{\prime}=w b$. Let $F$ and $F^{\prime}$ be the forks built by $\mathcal{A}^{*}$ for the characteristic string $w$ and $w b$, respectively, so that $F \sqsubseteq F^{\prime}$. By assumption, $F$ is a canonical fork for $w$; this means $\rho(F)=\rho(w)$ and for all $x \prec w, \mu_{x}(F)=\mu_{x}(y)$. It will be helpful for the reader to recall Fact 5 before proceeding.

Proving (17). We wish to show that $\rho\left(F^{\prime}\right)$ satisfies (17). If $b=\mathrm{A}$ then, by construction, $F^{\prime}=F$. The symbol $b=\mathrm{A}$ increases the reserve of every tine by one. Thus $\rho\left(F^{\prime}\right)=\rho(F)+1=\rho(x y)+1$. Now suppose $b \in\{\mathrm{h}, \mathrm{H}\}$. Since all tines $\sigma \in F^{\prime}$ with label $|x y|+1$ are conservative extensions, $\operatorname{reach}_{F^{\prime}}(\sigma)=0$ and the $F^{\prime}$-reach of all $F$-tines decreases by one. Let $t$ be a maximum-reach tine in $F$; since $F$ is canonical, $\operatorname{reach}_{F}(t)=\rho(F)=\rho(x y)$. Therefore, $\rho\left(F^{\prime}\right) \geq \operatorname{reach}_{F^{\prime}}(t)=\operatorname{reach}_{F}(t)-1=\rho(x y)-1$. If $\rho(F)=0$ then this inequality can be tightened, as follows. As all $F$-tines have negative $F^{\prime}$-reach, any maximum-reach $F^{\prime}$-tine must be one of the extensions; it follows that $\rho\left(F^{\prime}\right)=0$. Thus we have proved [17).

Proving (18). Let $w=x y$ be an arbitrary decomposition; this $x$ remains fixed for the remainder of the proof. (Note that $\mathcal{A}^{*}$ is unaware of this decomposition.)

Let $\tau_{x}, \tau_{\rho x} \in F^{\prime}$ be two $y b$-disjoint tines so that $\operatorname{reach}_{F^{\prime}}\left(\tau_{\rho x}\right)=\rho\left(F^{\prime}\right), \operatorname{reach}_{F^{\prime}}\left(\tau_{x}\right)=\mu_{x}\left(F^{\prime}\right)$, and, of all $y b$ disjoint tine pairs in $F^{\prime}$ that attain this requirement, these two tines diverge the earliest. We say that the tines $\tau_{x}, \tau_{\rho x}$ witness $\mu_{x}\left(F^{\prime}\right)$.

Designate the witness tines $t_{x}, t_{\rho x} \in F$ in the same way as we have designated $\tau_{x}, \tau_{\rho x} \in F^{\prime}$; specifically, $w, y$, and $F$ would substitute $w^{\prime}, y b$, and $F^{\prime}$ in the recipe above. By assumption, $F$ is a canonical fork for $x y$. Therefore, $\rho(F)=\operatorname{reach}_{F}\left(t_{\rho x}\right)=\rho(x y), t_{x}$ is $y$-disjoint with $t_{\rho x}$, and $\mu_{x}(F)=\operatorname{reach}_{F}\left(t_{x}\right)=\mu_{x}(y)$. We wish to show that $\mu_{x}\left(F^{\prime}\right)$ satisfies (18).

If $b=\mathrm{A}$ then, by construction, $F^{\prime}=F$ and, therefore, $t_{x}$ and $t_{\rho x}$ are $y b$-disjoint in $F^{\prime}$. Note that the $F^{\prime}$-reach of every $F$-tine is one plus its $F$-reach. Therefore, $\mu_{x}\left(F^{\prime}\right) \geq \min \left(\operatorname{reach}_{F^{\prime}}\left(t_{\rho x}\right), \operatorname{reach}_{F^{\prime}}\left(t_{x}\right)\right)=\operatorname{reach}_{F^{\prime}}\left(t_{x}\right)=$ $\operatorname{reach}_{F}\left(t_{x}\right)+1=\mu_{x}(y)+1$.

If $b \in\{\mathrm{h}, \mathrm{H}\}$, all tines in $F^{\prime}$ with label $|w|+1$ arise from conservative extensions. Since the tines $t_{x}, t_{\rho x}$ are $y b$-disjoint in $F^{\prime}$, it follows that $\mu_{x}\left(F^{\prime}\right) \geq \min \left(\operatorname{reach}_{F^{\prime}}\left(t_{x}\right), \operatorname{reach}_{F^{\prime}}\left(t_{\rho x}\right)\right) \geq \operatorname{reach}_{F^{\prime}}\left(t_{x}\right)=\operatorname{reach}_{F}\left(t_{x}\right)-1=$ $\mu_{x}(y)-1$. Here, the first inequality follows from the definition of relative margin and the second one from the fact that reach $\left(t_{x}\right) \leq \operatorname{reach}\left(t_{\rho x}\right)$ by assumption. The first equality follows from Fact 5 and the second one follows from our assumption that the tines $t_{\rho x}, t_{x} \in F$ witness $\mu_{x}(F)=\mu_{x}(y)$.

However, we can tighten the above inequality when $\mu_{x}(y)$ is zero, as follows. Recall the sets $Z, S, R$, the zeroreach tine $z_{1}$, and the maximum-reach tine $r_{1}$ from Figure 4 Also recall that $z_{1}$, of all zero-reach tines, diverges earliest from any maximum-reach tine. As $\operatorname{reach}_{F}\left(z_{1}\right)=\mu_{x}(F)=\mu_{x}(y)=0$, it follows that $z_{1}$ and $r_{1}$ must be $y$-disjoint. Let $\sigma_{1} \in F^{\prime}$ be the conservative extension of $z_{1}$.

If $\rho(x y) \geq 1$ and $\mu_{x}(y)=0$ then $\sigma_{1}$ is the only new extension in $F^{\prime}$ and it has reach zero in $F^{\prime}$. Note that $\operatorname{reach}_{F^{\prime}}\left(r_{1}\right)=$ $\operatorname{reach}_{F}\left(r_{1}\right)-1=\rho(F)-1 \geq 0$ since $\rho(F)=\rho(x y) \geq 1$ by assumption. It follows that $\mu_{x}\left(F^{\prime}\right) \geq$ $\min \left(\operatorname{reach}_{F^{\prime}}\left(\sigma_{1}\right), \operatorname{reach}_{F^{\prime}}\left(r_{1}\right)\right) \geq \operatorname{reach}_{F^{\prime}}\left(\sigma_{1}\right)=0$.

If $\rho(x y)=0$ and $\mu_{x}(y)=0$ then $Z=R$ and $|Z| \geq 2$. If $b=\mathrm{h}, \sigma_{1}$ is the only tine in $F^{\prime}$ with the maximum reach, zero. Note that reach $F_{F^{\prime}}\left(r_{1}\right)=\operatorname{reach}_{F}\left(r_{1}\right)-1=\rho(F)-1 \geq-1$. Since $\sigma_{1}$ and $r_{1}$ are $y b$-disjoint, it follows that $\mu_{x}\left(F^{\prime}\right) \geq \min \left(\operatorname{reach}_{F^{\prime}}\left(\sigma_{1}\right), \operatorname{reach}_{F^{\prime}}\left(r_{1}\right)\right) \geq \operatorname{reach}_{F^{\prime}}\left(r_{1}\right) \geq=-1$.

On the other hand, if $b=\mathrm{H}$ then $F^{\prime}$ contains two new conservative extensions, $\sigma_{1}$ and $\sigma_{2}$, both with label $|x y|+1$, where $z_{1} \prec \sigma_{1}$ and $r_{1} \prec \sigma_{2}$. These extensions, therefore, are $y b$-disjoint and have zero reach. It follows that $\mu_{x}\left(F^{\prime}\right) \geq 0$. 
Note that if we want (18) to hold only for a given prefix $x \preceq w$ (a scenario pertinent in [4]), the adversary $\mathcal{A}^{*}$ (which produces a canonical fork) would be an overkill. Instead, we can use a simpler, prefix-aware adversary such as the one mentioned at the outset of Section 6.5 , let us call this strategy $\mathcal{A}$. In addition, instead of assuming Theorem 6 . it suffices to assume Proposition 2 inductively for all strings of length $|w|$. Let $F$ be the fork built by $\mathcal{A}$ for the string $w=x y$. In conjunction with Proposition 1 this would imply " $\rho(F)=\rho(w)$ and $\mu_{x}(F)=\mu_{x}(y)$," a critical property used inside the above proof. We omit further details.

\subsection{Proof of Theorem 5 and Theorem 6}

Proof of Theorem[5. Let $w \in\{\mathrm{h}, \mathrm{H}, \mathrm{A}\}^{*}$. If $w=\varepsilon$ then, by Claim $3 \rho(\varepsilon)=0$. If $|w| \geq 1$, (13) is implied by the combination of Claim 3 , (15) and (17).

Let $w=x y$ be an arbitrary decomposition. We proceed by induction on $|y|$. If $|y|=0$ then Claim[3implies that $\mu_{x}(\varepsilon)=\rho(x)$. Otherwise, (14) is implied by the combination of Claim 3 (16) and (18).

Proof of Theorem 6. The proof is by induction on $|w|$. If $w$ is the empty string $\varepsilon$, the only fork $F \vdash \varepsilon$ is the trivial fork containing a single (honest) root vertex. By Claim 3 satisfies $\rho(\varepsilon)=0$ and $\mu_{\varepsilon}(\varepsilon)=\rho(\varepsilon)=0$.

Now, let $n$ be a non-negative integer and let $w$ be a characteristic string of length $n+1$. Assume that Theorem 6 holds for all characteristic strings of length $0,1, \ldots, n$. Note that this assumption satisfies the premise in Proposition 2 A combined application of Claim 3 . Proposition 1 and Proposition 2 implies Theorem 6 for $|w|=n+1$.

\section{The semi-synchronous setting}

We set the stage by stating the $\Delta$-synchronous model.

Definition 20 (Semi-synchronous characteristic string). Let $s l_{1}, \ldots, s l_{n}$ be a sequence of slots. A semi-synchronous characteristic string $w$ is an element of $\{\mathrm{h}, \mathrm{H}, \mathrm{A}, \perp\}^{n}$ defined for a particular execution of a blockchain protocol on these slots so that for $t \in[n], w_{t}=\perp$ if $s_{t}$ was assigned to no participants; otherwise, $w_{t}=\mathrm{A}$ if $\mathrm{sl}_{t}$ was assigned to an adversarial participant; otherwise, $w_{t}=\mathrm{h}$ if sl was assigned to a single honest participant; otherwise $w_{t}=\mathrm{H}$.

In the $\Delta$-synchronous setting, axiom $\mathbf{A 4}$ is replaced by

$\mathbf{A 4}_{\Delta}$. In a $\Delta$-synchronous execution, if two honestly generated blocks $B_{1}$ and $B_{2}$ are labeled with slots $s l_{1}$ and $s l_{2}$ for which $s l_{1}+\Delta<s l_{2}$, the length of the unique blockchain terminating at $B_{1}$ is strictly less than the length of the unique blockchain terminating at $B_{2}$.

Definition 21 ( $\Delta$-Fork). Let $w \in\{\mathrm{h}, \mathrm{H}, \mathrm{A}, \perp\}^{n}, \Delta \in\{0,1,2, \ldots\}, P=\left\{i: w_{i}=\mathrm{h}\right\}$, and $Q=\left\{j: w_{j}=\mathrm{H}\right\}$. A $\Delta$-fork for the semi-synchronous string $w$ consists of a directed and rooted tree $F=(V, E)$ with a labeling $\ell: V \rightarrow$ $\{0,1, \ldots, n\}$. We insist that each edge of $F$ is directed away from the root vertex. We require conditions (F1) (F3) from Definition 2 and

$\left(F 4_{\Delta}\right)$ for any indices $i, j \in P \cup Q$, if $i+\Delta<j$ then the depth of a vertex with label $i$ is strictly less than the depth of a vertex with label $j$.

If $F$ is a $\Delta$-fork for the semi-synchronous characteristic string $w$, we write $F \vdash_{\Delta} w$. A $\Delta$-fork generalizes a synchronous fork in Definition 2 2 since the latter is a $\Delta$-fork with $\Delta=0$. We sometimes emphasize this fact by writing $F^{\prime} \vdash_{0} w^{\prime}$ where $w^{\prime}$ is a synchronous characteristic string and $F^{\prime}$ is a synchronous fork. Note that condition (F4 $)$ is a direct analogue of axiom $\mathbf{A 4}_{\Delta}$ (We already know that conditions (F1) (F3) are direct analogues of axioms $\mathbf{A 1}$ - $\mathbf{A 3}$ ) 
Definition 22 (Reduction map). For $\Delta \in \mathbb{N}$, we define the function $\rho_{\Delta}:\{\perp, \mathrm{h}, \mathrm{H}, \mathrm{A}\}^{*} \rightarrow\{\mathrm{h}, \mathrm{H}, \mathrm{A}\}^{*}$ inductively as follows: $\rho_{\Delta}(\varepsilon)=\varepsilon$ and for $w \in\{\perp, \mathrm{h}, \mathrm{H}, \mathrm{A}\}^{*}$,

$$
\rho_{\Delta}(b w)= \begin{cases}\rho_{\Delta}(w) & \text { if } b=\perp, \\ b \rho_{\Delta}(w) & \text { if } b \in\{\mathrm{h}, \mathrm{H}\} \text { and }\{\perp, \mathrm{A}\}^{\Delta} \preceq w, \\ \mathrm{~A} \rho_{\Delta}(w) & \text { otherwise. }\end{cases}
$$

Note that in the above definition, if $w^{\prime}=\rho_{\Delta}(w)$ and $A=\left\{i: w_{i} \neq \perp\right\}$ then $|A|=\left|w^{\prime}\right|$. Also note that the reduction $\rho_{\Delta}$ implicitly defines, for each $w$, a bijective, increasing function $\pi: A \rightarrow\left[\left|w^{\prime}\right|\right]$. Note that $\rho_{\Delta}$ turns an $\mathrm{h}$ or $\mathrm{H}$ symbol in $w$ into an A symbol in $w^{\prime}$ with a constant probability. Therefore, for any slot $t$ in $w$, the reduction map $\rho_{\Delta}$ amplifies the probability that the slot $\pi(t)$ in $w^{\prime}=\rho_{\Delta}(w)$ is adversarial.

Definition $23(\Delta$-settlement with parameters $s, k \in \mathbb{N})$. Let $n \in \mathbb{N}$ and let $w \in\{\perp, \mathrm{h}, \mathrm{H}, \mathrm{A}\}^{n}$. Let $t \in[s+k, n]$ be an integer, $\hat{w} \preceq w,|\hat{w}|=t$, and let $F$ be any $\Delta$-fork for $\hat{w}$. We say that a slot $s$ is not $(k, \Delta)$-settled in $F$ if $F$ contains two maximum-length tines $\mathcal{C}_{1}, \mathcal{C}_{2}$ so that at least one of these tines contains a vertex with label $s$, both tines contain at least $k$ vertices after slot $s$, and the label of their last common vertex is at most $s-1$. Otherwise, we say that slot $s$ is $(k, \Delta)$-settled in $F$. We say that slot $s$ is $(k, \Delta)$-settled in $w$ if, for each $t \geq s+k$, it is $(k, \Delta)$-settled in every $\Delta$-fork $F \vdash \hat{w}$ where $\hat{w} \preceq w,|\hat{w}|=t$.

Note that in the above definition, we truncated $k$ trailing blocks from a tine whereas in Definition 3 we truncated from a tine all trailing blocks corresponding to the last $k$ slots. Note that this change of perspective is necessary since $w$ may contain $\perp$ symbols, i.e., empty slots.

Theorem 7 (Main theorem; $\Delta$-synchronous setting). Let $f, \epsilon \in(0,1)$ and $\Delta \in\{0,1,2, \ldots\}$. Let $s, k, T \in \mathbb{N}$ so that $T \geq s+k+\Delta$. Write $p_{\perp}=1-f$ and $\beta=(1-f)^{\Delta}$. Let $p_{\mathrm{A}} \in[0, f)$ so that $p_{\mathrm{A}}, f, \epsilon$, and $\beta$ satisfy

$$
p_{\mathrm{A}} \beta / f+(1-\beta) \leq(1-\epsilon) / 2 .
$$

Let $p_{\mathrm{h}} \in\left(0, f-p_{\mathrm{A}}\right]$ and write $p_{\mathrm{H}}=f-p_{\mathrm{A}}-p_{\mathrm{h}}$. Let $w \in\{\perp, \mathrm{h}, \mathrm{H}, \mathrm{A}\}^{T}$ be a random variable so that each $w_{i}, i \in[T]$, is independent and identically distributed as follows: $\operatorname{Pr}\left[w_{i}=\sigma\right]=p_{\sigma}$ for $\sigma \in\{\mathrm{h}, \mathrm{H}, \mathrm{A}, \perp\}$. Let $\mathcal{B}$ be the distribution of $w$. Then

$$
\underset{w}{\operatorname{Pr}}[\text { slot } s \text { is not }(k, \Delta) \text {-settled in } w] \leq \exp \left(-k \cdot \Omega\left(\min \left(\epsilon^{3}, \epsilon^{2} p_{\mathrm{h}} \beta / f\right)\right)+\frac{\epsilon(1+\Delta)}{1-\epsilon}\right) .
$$

(Here, the asymptotic notation hides constants that do not depend on $\epsilon$ or $k$.)

The main observation for proving the theorem above is that a $\Delta$-settlement violation in $w$, implies a certain combinatorial event (parameterized by $\Delta$ ) in a prefix of $\rho_{\Delta}(w)$. Specifically, we can analyze the latter event using techniques developed in proving Theorem 1

A comment on consistent chain selection. Assuming axiom ${\mathbf{A 0 ^ { \prime }}}^{\prime}$ is satisfied, it is easy to prove an analogue of Theorem 2 in the $\Delta$-synchronous setting; we need only use Bound2 in lieu of Bound 1 The resulting bound on the probability of a $(k, \Delta)$-settlement violation would be

$$
\exp \left(-k \cdot \Omega\left(\epsilon^{3}\right)+\frac{\epsilon(1+\Delta)}{1-\epsilon}\right) .
$$

We omit further details.

Road-map for the proof. Let $w \in\{\perp, \mathrm{h}, \mathrm{H}, \mathrm{A}\}^{*}, w^{\prime}=\rho_{\Delta}(w), n=|w|$, and $m=\left|\rho_{\Delta}\right|$. Our roadmap forward is as follows:

1. Show that there is a bijection between $\Delta$-forks for $w$ and synchronous forks for $w^{\prime}$. In particular, for each $\Delta$ fork $F \vdash_{\Delta} w$ there is an isomorphic synchronous fork $F^{\prime} \vdash_{0} w^{\prime}$ and a bijective map $\left\{i \in[n]: w_{i} \neq \perp\right\} \rightarrow[m]$. This is shown in Proposition 3 
2. Show that if $w$ violates $\Delta$-settlement then some prefix $b \prec \rho_{\Delta}(w)$ violates a suitably-defined combinatorial event $B_{\Delta}$. It is important that we can analyze this event using the techniques and results we have already established. This is done in Lemma2

3. Since the decisions made by $\rho_{\Delta}$ at each slot depends on the $\Delta$ future slots, the distribution of the last few symbols of $\rho_{\Delta}(w)$ will be "distorted" no matter how $w$ is distributed. Assuming $w$ has i.i.d. symbols, we need to show that the symbols in the aforementioned prefix $b \prec \rho_{\Delta}(w)$ are i.i.d. as well. This is done in Lemma 4

4. Obtain a bound on $\operatorname{Pr}\left[B_{\Delta}\right]$ in Bound 3 and proceed to prove Theorem 7

\subsection{Structural properties of the reduction map}

An important property of the reduction $w^{\prime}=\rho_{\Delta}(w)$ is that it readily provides a bijection between $\Delta$-forks for $w$ and synchronous forks for $w^{\prime}$.

Proposition 3. Let $w \in\{\perp, \mathrm{h}, \mathrm{H}, \mathrm{A}\}^{*}$ and $w^{\prime}=\rho_{\Delta}(w)$. Then, for every $\Delta$-fork $F \vdash w$ there is a synchronous fork $F^{\prime} \vdash_{0} w^{\prime}$ which is isomorphic to $F$. $F^{\prime}$ is called the image of $F$ under $\rho_{\Delta}$.

Proof sketch. Let $F^{\prime}$ be a copy of $F$. Establish the natural bijection $m: V(F) \rightarrow V\left(F^{\prime}\right)$ given by the copying proess, i.e., $u \mapsto m(u)$, and relabel the vertices as

$$
\ell(m(u))=\pi(\ell(u)) \text { for each vertex } u \in F .
$$

Set $r\left(F^{\prime}\right)=m(r(F))$ and $\ell\left(r\left(F^{\prime}\right)\right)=0$. It suffices to check that $F^{\prime} \vdash_{0} w^{\prime}$, i.e., $F^{\prime}$ is a valid (synchronous) fork for $w^{\prime}$. Specifically, if there are two honest slots $h_{1}, h_{2}$ in $w$ within a distance $\Delta$ of each other, then the former honest slot is mapped to an adversarial slot in $w^{\prime}$. Therefore, in $F^{\prime}$, an honest vertex is aware of all honest vertices with smaller labels.

Next, we show that a $\Delta$-settlement violation in $w$ implies a combinatorial event in $\rho_{\Delta}(w)^{\lfloor\Delta} \in\{\mathrm{h}, \mathrm{H}, \mathrm{A}\}^{*}$. It follows that we can use our existing stochastic techniques to bound $\Delta$-settlement violations on $w$.

Let $w^{\prime} \in\{\mathrm{h}, \mathrm{H}, \mathrm{A}\}^{*}$ be a characteristic string. Define $b_{i} \in\{ \pm 1\}$ as $b_{i}=1$ iff $w_{i}^{\prime}=\mathrm{A}$. Let $S=\left(S_{i}\right)_{i=0}^{\left|w^{\prime}\right|}$ be a simple biased walk on $\mathbb{Z}$ defined as $S_{0}=0, S_{i}=S_{i-1}+b_{i}$.

Lemma 2. Let $w \in\{\perp, \mathrm{h}, \mathrm{H}, \mathrm{A}\}^{*}, \Delta, s, k \in \mathbb{N}$ so that $|x|=s$ and $x_{s} \neq \perp$. Let $w^{\prime}=\rho_{\Delta}(w)$ and write $w^{\prime}=x^{\prime} y^{\prime} z^{\prime} a^{\prime}$ so that $\left|a^{\prime}\right|=\Delta$ and $\left|y^{\prime}\right| \geq 2 k$. Recall the simple biased walk $S=\left(S_{i}\right)$ on $w^{\prime}$ defined above. Let $E$ denote the event that a slot $c^{\prime}$ in $y^{\prime}$ is Catalan in $x^{\prime} y^{\prime} z^{\prime}$ and $S_{c^{\prime}+k+i} \leq S_{c^{\prime}}-\Delta$ for all $i \geq 0$. If $E$ occurs then $s$ is $\left(\left|y^{\prime}\right|, \Delta\right)$-settled in $w$.

Proof. Let $\pi$ be the bijection described after Definition 22 Note that $\left|x^{\prime}\right|=\pi(s)$. Assume that $E$ occurs. Thus $y^{\prime}$ contains a uniquely honest slot $c^{\prime}$ which is Catalan in $x^{\prime} y^{\prime} z^{\prime}$. Note that $S_{\left|w^{\prime}\right|} \leq S_{\left|x^{\prime} y^{\prime} z^{\prime}\right|}+\Delta \leq\left(S_{c}-\Delta\right)+\Delta \leq S_{c^{\prime}}$ where the second inequality follows from the assumption that $E$ occurs. It follows that $c^{\prime}$ is Catalan in $w^{\prime}$ as well. Therefore, by Theorem $3, c^{\prime}$ has the UVP in $w^{\prime}$. Let $c$ be the integer satisfying $c^{\prime}=\pi(c)$.

Let $b \preceq x y z,|b| \geq|x y|$ and $b^{\prime}=\rho_{\Delta}(b) \preceq x^{\prime} y^{\prime} z^{\prime}$. (Necessarily, $\left|b^{\prime}\right| \geq\left|x^{\prime} y^{\prime}\right|$.) Since the reduction map gives an isomorphism between every $\Delta$-fork for $b$ and its unique image (which is a synchronous fork for $b^{\prime}$ ) under the reduction $\rho_{\Delta}$, it follows that $c$ has the UVP in $w$.

For any $\Delta$-fork $F \vdash_{\Delta} b$, let $u \in F, \ell(u)=c$ be the unique vertex contained by every tine $t \in F$ viable at the onset of any slot after $c$. Consider all tines $\tau \in F$ so that $\tau$ has at least $\left|y^{\prime}\right|$ vertices with label at least $s+1$. and $\tau$ is viable at the onset of $\operatorname{slot} \ell(\tau)+1$. Since $\ell(\tau) \geq|x y| \geq c$, it follows that $u \preceq \tau$. Thus all these tines $\tau$ agree about slot $s$ since $s<c=\ell(u)$. In particular, if $F$ contains two maximum-length tines $\tau_{1}, \tau_{2}$, each with at least $\left|y^{\prime}\right|$ vertices after slot $s$, then they would agree about slot $s$. In fact, $\ell\left(\tau_{1} \cap \tau_{2}\right) \geq c>s$. Hence $s$ must be $\left(\left|y^{\prime}\right|, \Delta\right)$-settled in $F$ and, since $F$ was arbitrary, $s$ must be $\left(\left|y^{\prime}\right|, \Delta\right)$-settled in $w$. 


\subsection{Stochastic properties of the reduction map}

It turns out that if the bits in $w$ are i.i.d. then so are the bits in a suitable prefix of $\rho_{\Delta}(w)$ albeit with a slightly different distribution (which accounts for the absence of the empty slots). Specifically, for any string $x=x_{1} x_{2} \ldots$ on any alphabet and any $k \in \mathbb{N}$, define $x^{\lfloor k} \triangleq x_{1} \ldots x_{|x|-k}$.

Proposition 4. Let $T \in \mathbb{N}, w=w_{1} \ldots w_{T} \in\{\perp, \mathrm{h}, \mathrm{H}, \mathrm{A}\}^{T}$ be a sequence of i.i.d. symbols, and define $p_{\sigma} \triangleq \operatorname{Pr}\left[w_{1}=\right.$ $\sigma]$ for each $\sigma \in\{\perp, \mathrm{h}, \mathrm{H}, \mathrm{A}\}$. Let $x=\rho_{\Delta}(w)$ and let $\ell=|x|$. Write $f=1-p_{\perp}$ and $\alpha=(1-f)^{\Delta}$. Then the symbols in the string $x^{\lfloor\Delta}$ are i.i.d. with

$$
\begin{aligned}
& \operatorname{Pr}\left[x_{i}=\mathrm{h}\right]=p_{\mathrm{h}} \cdot \alpha / f, \\
& \operatorname{Pr}\left[x_{i}=\mathrm{H}\right]=p_{\mathrm{H}} \cdot \alpha / f, \quad \text { and } \\
& \operatorname{Pr}\left[x_{i}=\mathrm{A}\right]=1-\alpha+p_{\mathrm{A}} \cdot \alpha / f
\end{aligned}
$$

for each $i \in[\ell-\Delta]$.

Proof. First let us pretend for a moment that $T=\infty$; then $\ell=\infty$ as well. Let us write the infinite sequence $w$ as a concatenation of segments of $\perp$ s punctuated by a single non- $\perp$ symbol. That is, write $w=b_{0} e_{1} b_{1} e_{2} b_{2} \ldots$ where, for $i=0,1, \ldots, b_{i}=\perp^{*}$ and $e_{i} \in\{\mathrm{h}, \mathrm{H}, \mathrm{A}\}$. The reduction map $\rho_{\Delta}$ translates a segment $e_{i} b_{i}$ into a symbol $z_{i}$ as follows:

$$
z_{i}= \begin{cases}\mathrm{A} & \text { if } e_{i}=\mathrm{A} \text { or }\left|b_{i}\right| \leq \Delta-1 \\ e_{i} & \text { if } e_{i} \in\{\mathrm{h}, \mathrm{H}\} \text { and }\left|b_{i}\right| \geq \Delta\end{cases}
$$

In particular, the segments $e_{i} b_{i}$ as well as the events that determine the value of an $z_{i}$ are disjoint. Therefore, the symbols in the infinite sequence $z_{1} z_{2} \ldots=\rho_{\Delta}\left(w_{1} w_{w} \ldots\right)$ are independent and identically distributed.

If $T$ is finite, however, the last $\Delta$ symbols of $x=\rho_{\Delta}(w)$ are "distorted" in that the translated symbols in this region will be more favored to be As. However, since the last $\Delta$ symbols of $x$ must correspond to at least $\Delta$ trailing symbols of $w$, it follows that $x_{1} \ldots x_{\ell-\Delta}$ is a prefix of $z_{1} z_{2} \ldots$

It remains to compute the probabilities. Let $q_{\sigma}=\operatorname{Pr}\left[z_{i}=\sigma\right]$ for any $i$ and $\sigma \in\{\mathrm{h}, \mathrm{H}, \mathrm{A}\}$. Then $q_{\mathrm{h}}=p_{\mathrm{h}} /(1-$ $\left.p_{\perp}\right) p_{\perp}^{\Delta}=p_{\mathrm{h}} \alpha / f, q_{\mathrm{H}}=p_{\mathrm{H}} \alpha / f$, and $q_{\mathrm{A}}=1-\left(q_{\mathrm{h}}+q_{\mathrm{H}}\right)=1-\left(p_{\mathrm{h}}+p_{\mathrm{H}}\right) \alpha / f=1-\left(f-p_{\mathrm{A}}\right) \alpha / f=1-\alpha+p_{\mathrm{A}} \alpha / f$.

The final ingredient to proving Theorem 7 is a tail bound for (the complement of) the event $E$ in Lemma 2

Bound 3. Let $T, s, k \in \mathbb{N}, T \geq s+2 k+\Delta$ and $\epsilon, q_{\mathrm{h}} \in(0,1)$ so that the characteristic string $w^{\prime} \in\{\mathrm{h}, \mathrm{H}, \mathrm{A}\}^{T}$ satisfies the $\left(\epsilon, q_{\mathrm{h}}\right)$-Bernoulli condition. Write $w^{\prime}=x^{\prime} y^{\prime} z^{\prime}$ so that $y^{\prime}=w_{s} \ldots w_{s+2 k-1}$. Let $G$ denote the event that $w^{\prime}$ has a Catalan slot $c$ which belongs to $y_{1}^{\prime} \ldots y_{k}^{\prime}$. Condition on $G$. Let $\Delta \in \mathbb{N}$ and recall the simple biased random walk $S=\left(S_{i}\right)$ on $w^{\prime}$ defined above Lemma 2 Let $B_{\Delta}$ be the event that $S_{c+k+i} \geq S_{c}-\Delta$ for some $i \geq 0$. Then for large $k$,

$$
\operatorname{Pr}_{w}\left[B_{\Delta} \mid G\right] \leq \exp \left(-k \cdot \Omega\left(\epsilon^{2}\right)+\frac{\epsilon(1+\Delta)}{1-\epsilon}\right) .
$$

Proof. For simplicity, write $p=q_{\mathrm{A}}$, and $q=q_{\mathrm{h}}+q_{\mathrm{H}}$. Conditioned on $G, S_{c} \geq S_{c+i}$ for all $i \geq 1$. Let $y=$ $y^{\prime}[c+1: c+k]$ so that $|y|=k$. Moreover, $\#_{\mathrm{A}}(y) \leq \#_{\mathrm{h}}(y)+\#_{\mathrm{H}}(y)$. Let $f_{i}(k), i=0,1, \ldots$ be the probability that $S_{c+k}=S_{c}-i$. Thus we wish to upper-bound $f(\Delta, k) \triangleq \sum_{i=0}^{\Delta} f_{j}(k)$.

Write $a=E_{\mathrm{A}}(y)$ and $h=k-a$ and suppose $h-a=j$ for some $j=0,1,2, \ldots$ Hence, for a fixed $j$, we have $h=(k+j) / 2$ and $a=(k-j) / 2$. In addition, $k$ and $j$ has the same parity. Thus,

$$
\begin{aligned}
f_{j}(k) & =\left(\begin{array}{c}
k \\
(k+j) / 2
\end{array}\right) p^{(k-j) / 2} q^{(k+j) / 2}=\left(\begin{array}{c}
k \\
(k+j) / 2
\end{array}\right)(p q)^{k / 2}(q / p)^{j / 2} \leq\left(\begin{array}{c}
k \\
k / 2
\end{array}\right)(p q)^{k / 2}(q / p)^{j / 2} \\
& =O(1) \cdot \frac{2^{k}}{\sqrt{\pi k}} \cdot\left(1-\epsilon^{2}\right)^{k} 2^{-k} \cdot(q / p)^{j / 2}=O(1) \cdot \frac{\left(1-\epsilon^{2}\right)^{k / 2}}{\sqrt{k}} \cdot(q / p)^{j / 2}
\end{aligned}
$$

since $p=(1-\epsilon) / 2$ and $q=(1+\epsilon) / 2$. It follows that

$$
f(\Delta, k)=\sum_{j=0}^{\Delta} f_{j}(k) \leq \frac{O(1)}{\sqrt{k}} \cdot\left(1-\epsilon^{2}\right)^{k / 2} \sum_{j=0}^{\Delta}(q / p)^{j / 2} \leq \frac{O(1)}{\sqrt{k}} \cdot \exp \left(-k \epsilon^{2} / 2\right) \cdot(1+\Delta)(q / p)^{\Delta / 2}
$$


Since

$$
(q / p)^{1 / 2}=\left(\frac{1+\epsilon}{1-\epsilon}\right)^{1 / 2}=\left(1+\frac{2 \epsilon}{1-\epsilon}\right)^{1 / 2} \leq \exp (\epsilon /(1-\epsilon))
$$

we have

$$
f(\Delta, k) \leq \frac{O(1+\Delta)}{\sqrt{k}} \cdot \exp \left(-k \epsilon^{2} / 2+(1+\Delta) \epsilon /(1-\epsilon)\right) .
$$

Note that for fixed $\epsilon$ and $\Delta, f(\Delta, k)$ decreases geometrically in $k$. Thus $\operatorname{Pr}\left[B_{\Delta} \mid G_{c}\right]=\sum_{t \geq k} f(\Delta, t)$ is no more than the quantity in (23).

\subsection{Proof of Theorem 7}

The symbols in $w$ are independent and identically distributed. Write $w^{\prime}=\rho_{\Delta}(w), w^{\prime}=x^{\prime} y^{\prime} z^{\prime} a^{\prime},\left|a^{\prime}\right|=\Delta$ and $\left|y^{\prime}\right| \geq 1+\Delta$. Let $k$ be an integer so that $\left|y^{\prime}\right|=2 k$. Recall the random walk $S=\left(S_{i}\right)$ on $w^{\prime}$ defined above Lemma2 Let $G_{1}$ denote the (good) event that a slot $c^{\prime}$ in $y^{\prime}$ is Catalan in $x^{\prime} y^{\prime} z^{\prime}$. Let $G_{2}$ denote the (good) event that $S_{c^{\prime}+k+i} \leq S_{c^{\prime}}-\Delta$ for all $i \geq 0$. By Lemma $2 G_{1} \cap G_{2}$ implies $\bar{A}$. (Here, '- denotes the complement.) The contrapositive of the above statement gives us

$$
\operatorname{Pr}[A] \leq \operatorname{Pr}\left[\overline{G_{1}}\right]+\operatorname{Pr}\left[\overline{G_{2}} \mid G_{1}\right] .
$$

The terms on the right-hand side can be bounded from above using Bounds 1 and 3 respectively, provided the symbols in $x^{\prime} y^{\prime} z^{\prime}$ are i.i.d. with $\operatorname{Pr}\left[x_{1}^{\prime}=\mathrm{A}\right]=(1-\epsilon) / 2$. Let us check whether this condition holds. We have $f=1-p_{\perp}$ and $\alpha=(1-f)^{\Delta}$. Proposition 4 states that the symbols of $x^{\prime} y^{\prime} z^{\prime}$ are i.i.d. with distribution given by (22). For each $\sigma \in\{\mathrm{h}, \mathrm{H}, \mathrm{A}\}$ we write $p_{\sigma}^{\prime}=\operatorname{Pr}\left[x_{1}^{\prime}=\sigma\right]$.

The condition (20) can be equivalently stated as $1-\left(1-p_{\mathrm{A}} / f\right) \alpha=(1-\epsilon) / 2$. We check that $p_{\mathrm{A}}^{\prime}=1-\left(p_{\mathrm{h}}^{\prime}+p_{\mathrm{H}}^{\prime}\right)=$ $1-\left(p_{\mathrm{h}}+p_{\mathrm{H}}\right) \alpha / f=1-\left(f-p_{\mathrm{A}}\right) \alpha / f=1-\left(1-p_{\mathrm{A}} / f\right) \alpha=(1-\epsilon) / 2$ and, consequently, $p_{\mathrm{h}}^{\prime}+p_{\mathrm{H}}^{\prime}=(1+\epsilon) / 2$.

Hence we can directly apply Bound 3 on the terms in the right-hand side of (24) to conclude that

$$
\operatorname{Pr}[A] \leq \exp \left(-k\left(\Omega\left(\min \left(\epsilon^{3}, \epsilon^{2} q_{\mathrm{h}}\right)\right)\right)+\frac{\epsilon(1+\Delta)}{1-\epsilon}\right) .
$$

The claim involving the distribution $\mathcal{W}$ follows from the analogous claim in Theorem 1

\section{The common prefix property}

For the sake of simplicity, assume the synchronous communication model from Section 2.2 the $\Delta$-synchronous setting can be handled in the same way as delineated in Sections 8 and 8 .

The common prefix property with parameter $k$ asserts that, for any slot index $s$, if an honest observer at slot $s+k$ adopts a blockchain $\mathcal{C}$, the prefix $\mathcal{C}[0: s]$ will be present in every honestly-held blockchain at or after slot $s+k$. (Here, $\mathcal{C}[0: s]$ denotes the prefix of the blockchain $\mathcal{C}$ containing only the blocks issued from slots $0,1, \ldots, s$.)

We translate this property into the framework of forks. Consider a tine $t$ of a fork $F \vdash w$. The trimmed tine $t^{k k}$ is defined as the portion of $t$ labeled with slots $\{0, \ldots, \ell(t)-k\}$. For two tines, we use the notation $t_{1} \preceq t_{2}$ to indicate that the tine $t_{1}$ is a prefix of tine $t_{2}$.

Definition 24 (Common Prefix Property with parameter $k \in \mathbb{N}$ ). Let $w$ be a characteristic string. A fork $F \vdash w$ satisfies $k$-CP slot if, for all pairs $\left(t_{1}, t_{2}\right)$ of viable tines $F$ for which $\ell\left(t_{1}\right) \leq \ell\left(t_{2}\right)$, we have $t_{1}^{\lfloor k} \preceq t_{2}$. Otherwise, we say that the tine-pair $\left(t_{1}, t_{2}\right)$ is a witness to a $k$-CP ${ }^{\text {slot }}$ violation. Finally, $w$ satisfies $k$-CP ${ }^{\text {slot }}$ if every fork $F \vdash w$ satisfies $k$-CP ${ }^{\text {slot }}$.

If a string $w$ does not possess the $k$ - $\mathrm{CP}^{\text {slot }}$ property, we say that $w$ violates $k$ - $\mathrm{CP}^{\text {slot }}$. Observe that traditionally (cf. [6]), the truncated chain is defined in terms of deleting a suffix of (block-)length $k$ from $\mathcal{C}$. We denote this traditional version of the common prefix property as the $k$-CP property. Note, however, that a $k$-CP violation immediately implies a $k$-CP $\mathrm{CP}^{\text {slot }}$ violation; hence, bounding the probability of a $k$-CP ${ }^{\text {slot }}$ violation is sufficient to rule out both events. 
Connection with the UVP. Note that if $w$ admits a $k$-CP $\mathrm{CP}^{\text {slot }}$ violation, then there must be a fork $F$ containing two distinct viable tines $t_{1}, t_{2}, \ell\left(t_{1}\right) \leq \ell\left(t_{2}\right)$ so that $\ell\left(t_{1}\right)-\ell\left(t_{1} \cap t_{2}\right) \geq k+1$. Then $t_{1}$ must contain a vertex $v, \ell\left(t_{1} \cap t_{2}\right)<\ell(v) \leq \ell\left(t_{1}\right)-k$ so that $v$ does not belong to $t_{2}$. If every substring $x$ of $w$ with $|x| \geq k$, contained a

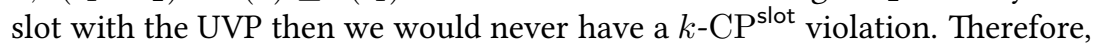

$$
\begin{gathered}
w \text { violates } \\
k \text {-CP }
\end{gathered} \Longrightarrow \begin{gathered}
w \text { has a substring } y,|y| \geq k \text { so } \\
\text { that no slot indexed by } y \text { has } \\
\text { the UVP in } w .
\end{gathered}
$$

Recall that a uniquely honest Catalan slot has the UVP. This fact allows us to bound the probability of common prefix violations by reasoning only about Catalan slots 5

Theorem 8 (Main theorem; CP version). Let $\epsilon, p_{\mathrm{h}} \in(0,1)$ and $T, k \in \mathbb{N}, T \geq k$. Let $w$ be a length-T characteristic string satisfying the $\left(\epsilon, p_{\mathrm{h}}\right)$-Bernoulli condition. Then

$$
\underset{w}{\operatorname{Pr}}[w \text { violates } k-\mathrm{CP}] \leq \operatorname{Pr}_{w}\left[w \text { violates } k-\mathrm{CP}^{\text {slot }}\right] \leq T \cdot \exp \left(-k \cdot \Omega\left(\min \left(\epsilon^{3}, \epsilon^{2} p_{\mathrm{h}}\right)\right)\right) .
$$

Next, suppose that axiom $\overline{A \boldsymbol{O}^{\prime}}$ is satisfied. If $w$ is a length-T bivalent characteristic string satisfying the $(\epsilon, 0)$ Bernoulli condition then

$$
\underset{w}{\operatorname{Pr}}[w \text { violates } k-\mathrm{CP}] \leq \operatorname{Pr}_{w}\left[w \text { violates } k-\mathrm{CP}^{\text {slot }}\right] \leq T \cdot \exp \left(-k \cdot \Omega\left(\epsilon^{3}(1+O(\epsilon))\right)\right)
$$

Proof. (The first claim.) Let $s \in[T-k]$. Let $\varepsilon_{k}$ be the probability that $y=w_{s} \ldots w_{s+k-1}$ contains no slot with the UVP in $w$. Then, recalling (25), we can apply a union bound over all substrings of $w$ of length at least $k$ to get $\operatorname{Pr}[w$ violates $k$-CP slot $] \leq T \sum_{r>k} \varepsilon_{r}$ where the factor $T$ represents a summation over all $s \in[T-k+1]$. By Theorem 3 if a substring $y$ of $w$ does not contain a slot with the unique vertex property in $w, y$ cannot contain a uniquely honest slot that is Catalan in $w$. Therefore, $\varepsilon_{k}$ is no more than the error probability from Bound 1 Since $\varepsilon_{k}$ decreases exponentially in $k$, we can write

$$
\operatorname{Pr}\left[w \text { violates } k \text {-CP }{ }^{\text {slot }}\right] \leq T \cdot O(1) \cdot \varepsilon_{k}
$$

This proves the second inequality. The first inequality follows since, in a given characteristic string, a $k$-CP violation implies a $k$-CP $\mathrm{CP}^{\text {slot }}$ violation.

(The second claim.) The proof in this case is identical to the preceding argument except that we need to refer to Theorem 4 in lieu of Theorem 3 and Bound 2 in lieu of Bound 1

The $\Delta$-synchronous setting. A $k$-CP violation in a $\Delta$-fork for a string $w \in\{\perp, \mathrm{h}, \mathrm{H}, \mathrm{A}\}^{*}$ would imply a $k$-CP violation in the corresponding synchronous fork in the string $\rho_{\Delta}(w) \in\{\mathrm{h}, \mathrm{H}, \mathrm{A}\}^{*}$ and, consequently, a $k$-CP $\mathrm{CP}^{\text {slot }}$ violation in $\rho_{\Delta}(w)$. We omit further details.

\section{Acknowledgments}

We thank Peter Gaži (IOHK) for finding a bug in Fact 1 in a previous version of this paper.

\section{References}

[1] Christian Badertscher, Peter Gaži, Aggelos Kiayias, Alexander Russell, and Vassilis Zikas. Ouroboros genesis: Composable proof-of-stake blockchains with dynamic availability. In Proceedings of the 2018 ACM SIGSAC Conference on Computer and Communications Security, CCS fi18, page 913fi?!930, 2018.

\footnotetext{
${ }^{5}$ One can also prove Theorem 8 by directly showing-as is done in [3]-that a $k$-CP ${ }^{\text {slot }}$ violation implies a $k$-settlement violation and then appealing to Theorem 1 The proof of the implication turns out to be quite long and complicated compared to the short proof above; see Appendix $\mathrm{A}$ A positive side of this this alternate proof, however, is that it shows how arguments in [3] can be adapted to our generalized fork framework.
} 
[2] Iddo Bentov, Rafael Pass, and Elaine Shi. Snow white: Provably secure proofs of stake. page 919, 2016. URL http://eprint.iacr.org/2016/919

[3] Erica Blum, Aggelos Kiayias, Cristopher Moore, Saad Quader, and Alexander Russell. Linear consistency for proof-of-stake blockchains. Technical report, Cryptology ePrint Archive, Report 2017/241, 2018. URL https://eprint.iacr.org/2017/241

[4] Erica Blum, Aggelos Kiayias, Cristopher Moore, Saad Quader, and Alexander Russell. The combinatorics of the longest-chain rule: Linear consistency for proof-of-stake blockchains. In Proceedings of the 2020 ACM Symposium on Discrete Algorithms, SODA '20, 2020.

[5] Bernardo David, Peter Gaži, Aggelos Kiayias, and Alexander Russell. Ouroboros praos: An adaptively-secure, semi-synchronous proof-of-stake blockchain. In Advances in Cryptology-EUROCRYPT 2018, pages 66-98, 2018.

[6] Juan Garay, Aggelos Kiayias, and Nikos Leonardos. The bitcoin backbone protocol with chains of variable difficulty. In Annual International Cryptology Conference, pages 291-323. Springer, 2017.

[7] Juan A. Garay and Aggelos Kiayias. Sok: A consensus taxonomy in the blockchain era. IACR Cryptology ePrint Archive, 2018:754, 2018. URL https://eprint.iacr.org/2018/754

[8] Aggelos Kiayias, Alexander Russell, Bernardo David, and Roman Oliynykov. Ouroboros: A provably secure proof-of-stake blockchain protocol. In Advances in Cryptology - CRYPTO 2017 - 37th Annual International Cryptology Conference, volume 10401 of Lecture Notes in Computer Science, pages 357-388, 2017.

[9] Silvio Micali. ALGORAND: the efficient and democratic ledger. CoRR, abs/1607.01341, 2016. URL http://arxiv.org/abs/1607.01341

[10] Rafael Pass and Elaine Shi. The sleepy model of consensus. In Advances in Cryptology-ASIACRYPT 2017, pages 380-409, 2017. URL https://doi.org/10 .1007/978-3-319-70697-9_14

[11] Saad Quader and Alexander Russell. C++ source code to compute settlement error estimates allowing concurrent honest slot leaders. https://github.com/saad0105050/multihonest-code 2020. Accessed: 2019-10-14.

[12] Herbert S Wilf. generatingfunctionology. AK Peters/CRC Press, 3 edition, 2005. 


\section{A CP violations and balanced forks with concurrent honest leaders}

Balanced forks played a critical role in the analysis of [3]. Specifically, a balanced fork was equivalent to a settlement violation in their setting and a $\mathrm{CP}$ violation would also imply a balanced fork. In the current analysis, we have analyzed settlement and CP violations through their connections with the UVP and Catalan slots; thus balanced forks are not necessary in our analysis. However, it is instructive to see whether the statement "a CP violation implies a balanced fork" still holds in our model and, importantly, how the existing proof needs to be modified.

Thus the the goal of this section is to prove Theorem 9 below which would yield an alternative proof of Theorem 8 without using the Catalan slots. However, the simplicity of the proof of Theorem 8 in Section 9 demonstrates the expressive power of the UVP and Catalan slots compared to relative margin and balanced forks.

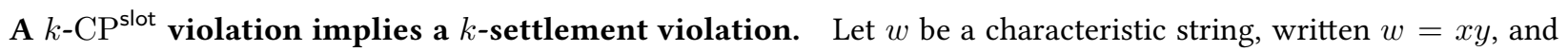
let $F$ be a fork for $w$. Recall that a slot $s=|x|+1$ is not $k$-settled if and only if $F$ contains two maximum-length tines that diverge prior to $s$, i.e., $F$ is $x$-balanced (see Definition 18).

Definition 25 (Slot divergence). Let $w \in\{\mathrm{h}, \mathrm{H}, \mathrm{A}\}^{*}$ and let $F$ be a fork for $w$. Define the slot divergence of two tines $t_{1}, t_{2} \in F$ as

$$
\operatorname{div}_{\text {slot }}\left(t_{1}, t_{2}\right) \triangleq \ell\left(t_{1}\right)-\ell\left(t_{1} \cap t_{2}\right) \quad \text { where } \ell\left(t_{1}\right) \leq \ell\left(t_{2}\right) .
$$

We can generalize this notion for forks and characteristic strings as follows: $\operatorname{div}_{\text {slot }}(F) \triangleq \max _{t_{1}, t_{2} \in F} \operatorname{div}_{\text {slot }}\left(t_{1}, t_{2}\right)$ and $\operatorname{div}_{\text {slot }}(w) \triangleq \max _{F \vdash w} \operatorname{div}_{\text {slot }}(F)$.

By definition, a $k-\mathrm{CP}^{\text {slot }}$ violation implies the existence of a fork with a slot divergence at least $k+1$. Theorem 9 below shows that a if a fork has a slot divergence at least $k+1$ then there is a balanced fork for a prefix of the same characteristic string so that two maximum-length tine diverge prior to last $k$ slots. Therefore, a $k$-CP ${ }^{\text {slot }}$ violation implies an $(s, k)$-settlement violation for some slot $s$.

Theorem 9. Let $k, T \in \mathbb{N}$. Let $w \in\{\mathrm{h}, \mathrm{H}, \mathrm{A}\}^{T}$ be a characteristic string so that $\operatorname{div}_{\text {slot }}(w) \geq k+1$. Then there is a decomposition $w=x y z$ and a fork $\hat{F} \vdash x y$, where $|y| \geq k$, so that $\hat{F}$ is $x$-balanced. define

Recall that $\ell(t)$ is the slot index of the last vertex of tine $t$. Define $A \triangleq \bigcup_{F \vdash w} A_{F}$ where, for a given fork $F \vdash w$,

$$
A_{F} \triangleq\left\{\left(\tau_{1}, \tau_{2}\right): \begin{array}{l}
\tau_{1}, \tau_{2} \text { are two viable tines in the fork } F, \\
\ell\left(\tau_{1}\right) \leq \ell\left(\tau_{2}\right), \text { and } \operatorname{div}_{\text {slot }}\left(\tau_{1}, \tau_{2}\right) \geq k+1
\end{array}\right\} .
$$

Notice that there must be a tine-pair $\left(t_{1}, t_{2}\right) \in A$ which satisfies the following two conditions:

$$
\operatorname{div}_{\text {slot }}\left(t_{1}, t_{2}\right) \text { is maximal over } A \text {, }
$$

$$
\left|\ell\left(t_{2}\right)-\ell\left(t_{1}\right)\right| \text { is minimal among all tine-pairs in } A \text { for which (27) holds, }
$$

and

For a fixed $t_{2}$, the tine $t_{1}$ has the maximum length over all tines $t_{1}^{\prime}, \ell\left(t_{1}^{\prime}\right)=\ell\left(t_{1}\right)$ such that $\left(t_{1}^{\prime}, t_{2}\right)$ satisfies (27) and (28).

(Note that $t_{1}, t_{2}$ are not uniquely identified.) The tines $t_{1}, t_{2}$ will play a special role in our proof; let $F$ be a fork containing these tines.

Recall given a characteristic string $w \in\{\mathrm{h}, \mathrm{H}, \mathrm{A}\}^{*}$, a uniquely honest slot contains the symbol $\mathrm{h}$, a multiply honest slot contains the symbol $\mathrm{H}$, and an adversarial slot contains the symbol A. We call a slot honest if it contains either an $\mathrm{h}$ or an $\mathrm{H}$; otherwise, we call it an adversarial slot.

The prefix $x$, fork $F_{x}$, and vertex $u$. Let $u$ denote the last vertex on the tine $t_{1} \cap t_{2}$, as shown in the diagram below, and let $\alpha \triangleq \ell(u)=\ell\left(t_{1} \cap t_{2}\right)$. Let $x \triangleq w_{1}, \ldots, w_{\alpha}$ and let $F_{x}$ be the fork-prefix of $F$ supported on $x$. We will argue that $\alpha$ must be a uniquely honest slot and, in addition, that $F_{x}$ must contain a unique longest tine $t_{u}$ terminating at the vertex $u$. We will also identify a substring $y,|y| \geq k$ such that $w$ can be written as $w=x y z$. Then we will construct a balanced fork $\tilde{F}_{y} \vdash y$ by modifying the subgraph of $F$ supported on $y$. We will finish the proof by constructing an $x$-balanced fork by suitably appending $\tilde{F}_{y}$ to $F_{x}$. 


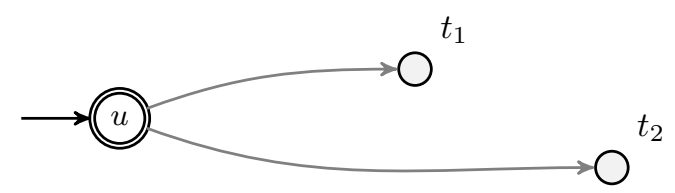

$\alpha$ must be a uniquely honest slot. We observe, first of all, that the slot $\alpha$ can neither be adversarial nor multiply honest: otherwise it is easy to construct a fork $F^{\prime} \vdash w$ and a pair of tines in $F^{\prime}$ that violate (27). Specifically, construct $F^{\prime}$ from $F$ by adding a new vertex $u^{\prime}$ to $F$ for which $\ell\left(u^{\prime}\right)=\ell(u)$, adding an edge to $u^{\prime}$ from the vertex preceding $u$, and replacing the edge of $t_{1}$ following $u$ with one from $u^{\prime}$; then the other relevant properties of the fork are maintained, but the slot divergence of the resulting tines has increased by at least one. (See the diagram below.)

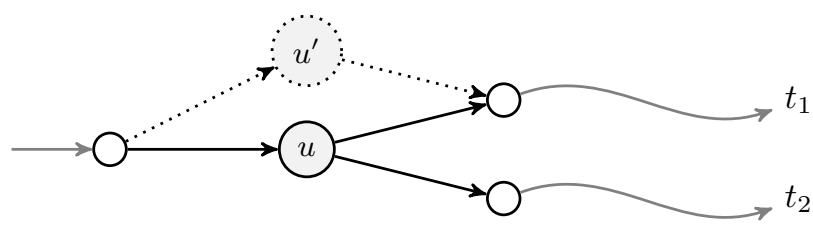

$F_{x}$ has a unique, longest (and honest) tine $t_{u}$. A similar argument implies that the fork $F_{x}$ has a unique vertex of depth $\operatorname{depth}(u)$ : namely, $u$ itself. In the presence of another vertex $u^{\prime}$ (of $F_{x}$ ) with depth $\operatorname{depth}(u)$, "redirecting" $t_{1}$ through $u^{\prime}$ (as in the argument above) would likewise result in a fork with a larger slot divergence. To see this, notice that $\ell\left(u^{\prime}\right)$ must be strictly less than $\ell(u)$ since $\ell(u)$ is an honest slot (which means $u$ is the only vertex at that slot). Thus $\ell(\cdot)$ would indeed be increasing along this new tine (resulting from redirecting $t_{1}$ ). As $\alpha$ is the last index of the string $x$, this additionally implies that $F_{x}$ has no vertices of depth exceeding $\operatorname{depth}(u)$. Let $t_{u} \in F_{x}$ be the tine with $\ell\left(t_{u}\right)=\alpha$.

The honest tine $t_{u}$ is the unique longest tine in $F_{x}$.

Identifying $y$. Let $\beta$ denote the smallest honest index of $w$ for which $\beta \geq \ell\left(t_{2}\right)$, with the convention that if there is no such index we define $\beta=T+1$. Thus $\beta \geq \ell\left(t_{2}\right) \geq \ell\left(t_{1}\right)$. These indices, $\alpha$ and $\beta$, distinguish the substrings $y=w_{\alpha+1} \ldots w_{\beta-1}$ and $z=w_{\beta} \ldots w_{T}$; we will focus on $y$ in the remainder of the proof. Since the function $\ell(\cdot)$ is strictly increasing along any tine, observe that

$$
|y|=(\beta-1)-(\alpha+1)+1=\beta-\alpha-1 \geq\left(\ell\left(t_{1}\right)-\ell(u)\right)-1 \geq(k+1)-1=k .
$$

Hence $y$ has the desired length and it suffices to establish that it is forkable 6

Honest indices in $x y$ have small depths. The minimality assumption (28) implies that any honest index $h$ for which $h<\beta$ has depth no more than $\min \left(\right.$ length $\left(t_{1}\right)$, length $\left.\left(t_{2}\right)\right)$ : specifically, we claim that

$$
h<\beta \quad \Longrightarrow \quad \mathbf{d}(h) \leq \min \left(\operatorname{length}\left(t_{1}\right), \operatorname{length}\left(t_{2}\right)\right) \text {. }
$$

To see this, consider an honest index $h, h<\beta$ and a tine $t_{h}$ for which $\ell\left(t_{h}\right)=h$. If $\ell\left(t_{2}\right)$ is honest then $h<\beta=\ell\left(t_{2}\right)$. Otherwise, $h<\ell\left(t_{2}\right)<\beta$ since $\ell\left(t_{2}\right)$ is adversarial. In any case, $h<\ell\left(t_{2}\right)$ and, since $t_{2}$ is viable, it follows immediately that $\mathbf{d}(h) \leq$ length $\left(t_{2}\right)$. Similarly, if $h<\ell\left(t_{1}\right)$ then $\mathbf{d}(h) \leq \operatorname{length}\left(t_{1}\right)$ since $t_{1}$ is viable as well.

Now consider the case $h=\ell\left(t_{1}\right)$. We claim that

$$
\text { If } h=\ell\left(t_{1}\right)<\beta \text { then } \mathbf{d}(h)=\operatorname{length}\left(t_{1}\right) .
$$

We can rule out the case $h=\ell\left(t_{1}\right)=\ell\left(t_{2}\right)$ since if this happens, $\ell\left(t_{2}\right)$ is honest and $\beta=\ell\left(t_{2}\right)$, contradicting our assumption that $h<\beta$. Thus, it must be the case that $h=\ell\left(t_{1}\right)<\ell\left(t_{2}\right)$. In this case, the claim follows trivially if

\footnotetext{
${ }^{6}$ In Blum et al. [3], $|y|$ was at least $k+1$. The difference is due to the fact that in their analysis, a slot with multiple vertices was necessarily adversarial.
} 
$\ell\left(t_{1}\right)$ is a uniquely honest slot. Otherwise, let $t$ be a tine with the maximum length among all tines labeled with the multiply honest slot $h=\ell\left(t_{1}\right)<\ell\left(t_{2}\right)$. We wish to show that length $\left(t_{1}\right)=$ length $(t)$. There are four contingencies to consider; the first three of these lead to contradictions and for the last one, we get length $\left(t_{1}\right)=\mathbf{d}(h)=\operatorname{length}(t)$.

- If $\left(t, t_{2}\right) \notin A$, $\operatorname{div}_{\text {slot }}\left(t, t_{2}\right)$ is at most $k$. Since $\operatorname{div}_{\text {slot }}\left(t_{1}, t_{2}\right)$ is at least $k+1, t$ must share a vertex with $t_{2}$ after slot $\ell(u)$. But this means $\ell\left(t \cap t_{1}\right)=\ell(u)$ and $\operatorname{div}_{\text {slot }}\left(t, t_{1}\right)=\operatorname{div}_{\text {slot }}\left(t_{1}, t_{2}\right) \geq k+1$. As a result, $\left(t, t_{1}\right) \in A$. However, this violates (28) since $\left|\ell(t)-\ell\left(t_{1}\right)\right|=0<\left|\ell\left(t_{2}\right)-\ell\left(t_{1}\right)\right|$ by assumption.

- If $\left(t, t_{2}\right)$ is in $A$ and $\ell\left(t \cap t_{1}\right)<\ell(u)$, then $\operatorname{div}_{\text {slot }}\left(t, t_{1}\right)>\operatorname{div}_{\text {slot }}\left(t_{1}, t_{2}\right)$, violating (27).

- If $\left(t, t_{2}\right)$ is in $A$ and $\ell\left(t \cap t_{1}\right)=\ell(u)$, this means $t$ is disjoint with $t_{1}$ after $\ell(u)$. Then (28) is violated since $\operatorname{div}_{\text {slot }}\left(t, t_{1}\right)=\operatorname{div}_{\text {slot }}\left(t_{1}, t_{2}\right)$ but $\left|\ell(t)-\ell\left(t_{1}\right)\right|=0<\left|\ell\left(t_{2}\right)-\ell\left(t_{1}\right)\right|$ by assumption.

- If $\left(t, t_{2}\right)$ is in $A$ and $\ell\left(t \cap t_{1}\right)>\ell(u)$, this means $t$ shares a vertex with $t_{1}$ after $\ell(u)$. Then $\operatorname{div}_{\text {slot }}\left(t, t_{2}\right)=$ $\operatorname{div}_{\text {slot }}\left(t_{1}, t_{2}\right)$ and $\left|\ell\left(t_{2}\right)-\ell\left(t_{1}\right)\right|=\left|\ell\left(t_{2}\right)-\ell(t)\right|$. By (29), length $\left(t_{1}\right) \geq \operatorname{length}(t)$; hence length $\left(t_{1}\right)=$ length $(t)$ since by assumption, $t$ has the maximum length among all tines with label $\ell\left(t_{1}\right)$. Hence length $\left(t_{1}\right)=\mathbf{d}(h)$.

The remaining case for proving (31), i.e., when $\ell\left(t_{1}\right)<h<\ell\left(t_{2}\right)$, can be ruled out by the argument below.

There is no honest index between $\ell\left(t_{1}\right)$ and $\ell\left(t_{2}\right)$. We claim that

$$
\text { There is no honest index } h \text { satisfying } \ell\left(t_{1}\right)<h<\ell\left(t_{2}\right) \text {. }
$$

The claim above is trivially true if $\ell\left(t_{1}\right)=\ell\left(t_{2}\right)$. Otherwise, suppose (toward a contradiction) that $h$ is an honest index satisfying $\ell\left(t_{1}\right)<h<\ell\left(t_{2}\right)$. Let $t_{h}$ be an honest tine at slot $h$. The tine-pair $\left(t_{1}, t_{h}\right)$ may or may not be in $A$. We will show that both cases lead to contradictions.

- If $\left(t_{1}, t_{h}\right)$ is in $A$ and $\ell\left(t_{1} \cap t_{h}\right) \leq \ell(u), \operatorname{div}_{\text {slot }}\left(t_{1}, t_{h}\right)$ is at least $\operatorname{div}_{\text {slot }}\left(t_{1}, t_{2}\right)$. In fact, due to (27), this inequality must be an equality. However, the assumption $\ell\left(t_{1}\right)<h<\ell\left(t_{2}\right)$ contradicts (28).

- If $\left(t_{1}, t_{h}\right)$ is in $A$ and $\ell\left(t_{1} \cap t_{h}\right)>\ell(u)$, it follows that $\operatorname{div}_{\text {slot }}\left(t_{h}, t_{2}\right)>\operatorname{div}_{\text {slot }}\left(t_{1}, t_{2}\right)$. As the latter quantity is at least $k+1,\left(t_{h}, t_{2}\right)$ must be in $A$. The preceding inequality, however, contradicts (27).

- If $\left(t_{1}, t_{h}\right) \notin A, \operatorname{div}_{\text {slot }}\left(t_{1}, t_{h}\right)$ is at most $k$. As $\operatorname{div}_{\text {slot }}\left(t_{1}, t_{2}\right)$ is at least $k+1, t_{h}$ and $t_{1}$ must share a vertex after slot $\ell(u)$. Since $\ell\left(t_{1}\right)<h<\ell\left(t_{2}\right)$ by assumption, $\operatorname{div}_{\text {slot }}\left(t_{h}, t_{2}\right)>\operatorname{div}_{\text {slot }}\left(t_{1}, t_{2}\right) \geq k+1$ and, as a result, $\left(t_{h}, t_{2}\right) \in A$. However, the strict inequality above violates (27).

We conclude that (33)-and thus (31)-is true. (Note that in the above argument, all we needed was that $t_{h}$ is a viable tine since in all cases, $t_{h}$ appears in a tine-pair in $A$. Thus (33) can be generalized as saying "there is no fork for $w$ with a viable tine $t$ so that $\ell\left(t_{1}\right)<\ell(t)<\ell\left(t_{2}\right)$.")

A fork $F^{\triangleright u \triangleleft}$ where all long tines go through $u$. In light of the remarks above, we observe that the fork $F$ may be "pinched" at $u$ to yield an essentially identical fork $F^{\triangleright u \triangleleft} \vdash w$ with the exception that all tines of length exceeding $\operatorname{depth}(u)$ pass through the vertex $u$. Specifically, the fork $F^{\triangleright u \triangleleft} \vdash w$ is defined to be the graph obtained from $F$ by changing every edge of $F$ directed towards a vertex of depth $\operatorname{depth}(u)+1$ so that it originates from $u$. To see that the resulting tree is a well-defined fork, it suffices to check that $\ell(\cdot)$ is still increasing along all tines of $F^{\triangleright u \triangleleft}$. For this purpose, consider the effect of this pinching on an individual tine $t$ terminating at a particular vertex $v$-it is replaced with a tine $t^{\triangleright u \triangleleft}$ defined so that:

- If length $(t) \leq \operatorname{depth}(u)$, the tine $t$ is unchanged: $t^{\triangleright u \triangleleft}=t$.

- Otherwise, length $(t)>\operatorname{depth}(u)$ and $t$ has a vertex $v$ of depth $\operatorname{depth}(u)+1$; note that $\ell(v)>\ell(u)$ because $F_{x}$ contains no vertices of depth exceeding $\operatorname{depth}(u)$. Then $t^{\triangleright u \triangleleft}$ is defined to be the path given by the tine terminating at $u$, a (new) edge from $u$ to $v$, and the suffix of $t$ beginning at $z$. (As $\ell(v)>\ell(u)$ this has the increasing label property.)

Thus the tree $F^{\triangleright u \triangleleft}$ is a legal fork on the same vertex set; note that the depths of vertices in $F$ and $F^{\triangleright u \triangleleft}$ are identical. 
Constructing a fork $F_{y} \vdash y$ containing two long tines. By excising the tree rooted at $u$ from this pinched fork $F^{\triangleright u \triangleleft}$, we may extract a fork for the string $w_{\alpha+1} \ldots w_{T}$. Specifically, consider the induced subgraph $F^{u \triangleleft}$ of $F^{\triangleright u \triangleleft}$ given by the vertices $\{u\} \cup\{v: \operatorname{depth}(v)>\operatorname{depth}(u)\}$. By treating $u$ as a root vertex and suitably defining the labels $\ell^{u \triangleleft}$ of $F^{u \triangleleft}$ so that $\ell^{u \triangleleft}(v)=\ell(v)-\ell(u)$, this subgraph has the defining properties of a fork for $w_{\alpha+1} \ldots w_{T}$. In particular, considering that $\alpha$ is honest, it follows that each honest index $h>\alpha$ has depth $\mathbf{d}(h)>\operatorname{length}(u)$ and hence any vertex with label $h$ is also present in $F^{u \triangleleft}$. For a tine $t$ of $F^{\triangleright u \triangleleft}$, we let $t^{u \triangleleft}$ denote the suffix of this tine beginning at $u$, which forms a tine in $F^{u \triangleleft}$. (If length $(t) \leq \operatorname{depth}(u)$, we define $t^{u \triangleleft}$ to consist solely of the vertex $u$.) Considering $t_{1} u \triangleleft$ and $t_{2}{ }^{u \triangleleft}$, let $\check{t}_{i}, i \in\{1,2\}$ be the longest prefix of $t_{i}{ }^{u \triangleleft}$ so that $\check{t}_{i}$ is labeled by a slot in $y$. Since the tines $t_{1}{ }^{u \triangleleft}, t_{2}{ }^{u \triangleleft}$ are disjoint in $F^{u \triangleleft}$, so are $\check{t}_{1}, \check{t}_{2}$.

Recall that that $y$ is as a prefix of $w_{\alpha+1} \ldots w_{T}$. Let $h^{*}$ be the largest honest index in $y$. Let $F_{y}$ denote the subtree of $F^{u \triangleleft}$, with the same root as $F^{u \triangleleft}$, containing the following tines: $\check{t}_{1}, \check{t}_{2}$, and all tines $t^{u \triangleleft} \in F^{u \triangleleft} \backslash\left\{\check{t}_{1}, \check{t}_{2}\right\}$ so that $\ell\left(t^{u \triangleleft}\right)$ is drawn from $y$ and

$$
\operatorname{length}\left(t^{u \triangleleft}\right) \leq \mathbf{d}\left(h^{*}\right) .
$$

Note that the length of every honest tine labeled by $y$ is at most $\mathbf{d}\left(h^{*}\right)$; hence, thanks to (31), $F_{y}$ contains all honest tines from $F^{u \triangleleft}$ that have labels in $y$. Note, in addition, that the tines $\check{t}_{1}$ and $\check{t}_{2}$ are consistently labeled in $F_{y}$. Thus $F_{y}$ satisfies all properties of a legal fork.

Having defined $F_{y}$, we claim that

$$
\min \left(\operatorname{length}\left(\check{t}_{1}\right), \operatorname{length}\left(\check{t}_{2}\right)\right) \geq \mathbf{d}\left(h^{*}\right) .
$$

Let $i \in\{1,2\}$. If $\ell\left(t_{i}\right)<\beta$ then $\check{t}_{i}=t_{i}{ }^{u \triangleleft}$ and, by (31), length $\left(\check{t}_{i}\right)=\operatorname{length}\left(t_{i}{ }^{u \triangleleft}\right) \geq \mathbf{d}\left(h^{*}\right)$. Othereise, we have $\ell\left(t_{i}\right)=\beta$ which means $\ell\left(t_{i}\right)$ is an honest slot. Thus $t_{i}{ }^{u \triangleleft}$ must be an honest tine, building directly on top of the viable tine $\check{t}_{i}$. Therefore, we have length $\left(\check{t}_{i}\right) \geq \mathbf{d}\left(h^{*}\right)$.

Constructing a balanced fork $\tilde{F}_{y} \vdash y$. If length $\left(\check{t}_{1}\right)=\operatorname{length}\left(\check{t}_{2}\right)$, set $\tilde{F}_{y}=F_{y}$ and, due to (34) and (35), the fork $\tilde{F}_{y} \vdash y$ must be balanced. Otherwise, let $a, b \in\{1,2\}, a \neq b$ be two integers so that length $\left(\check{t}_{a}\right)>\operatorname{length}\left(\check{t}_{b}\right)$. We modify $F_{y}$ by deleting some trailing nodes from $\tilde{t}_{a}$ so that the surviving prefix-let it be denoted by $\tilde{t}_{a}-$ has the same length as $\breve{t}_{b}$. That is, we achieve

$$
\operatorname{length}\left(\tilde{t}_{a}\right)=\operatorname{length}\left(\check{t}_{b}\right)=\min \left(\operatorname{length}\left(\check{t}_{1}\right), \operatorname{length}\left(\check{t}_{2}\right)\right) .
$$

Let $\tilde{F}_{y}$ be the resulting fork. Equations (34) and (35) imply that $\tilde{F}_{y}$ has at least two maximum-length tines (i.e., $\tilde{t}_{a}$ and $\left.\check{t}_{b}\right)$ and therefore, it is balanced. It remains to show that the longer tine, $\check{t}_{a}$, has sufficiently many trailing adversarial vertices so that after deleting them, we obtain length $\left(\tilde{t}_{a}\right)=\operatorname{length}\left(\check{t}_{b}\right)$. (If we had to delete an honest vertex in this process, $\tilde{F}_{y}$ may have violated property (F3) in the definition of a fork.) Let $h_{a}$ be the label of the last honest vertex on $\check{t}_{a}$. Thanks to (35), we have length $\left(\check{t}_{a}\right)>\operatorname{length}\left(\check{t}_{b}\right) \geq \mathbf{d}\left(h^{*}\right) \geq \mathbf{d}\left(h_{a}\right)$. Hence all vertices in $\check{t}_{a}$ with labels in $\left[h_{a}+1, \ell\left(\check{t}_{a}\right)\right]$ must be adversarial; we can safely delete $\mid$ length $\left(\check{t}_{a}\right)$ - length $\left(\check{t}_{b}\right) \mid$ of these adversarial vertices.

An $x$-balanced fork $\hat{F} \sqsubseteq F$. Let us identify the root of the fork $\tilde{F}_{y}$ with the vertex $u$ of $F_{x}$ and let $\hat{F}$ be the resulting graph (after "gluing" the root of $\tilde{F}_{y}$ to $u$ ). By $(\underline{30})$, it is easy to see that the fork $\hat{F} \sqsubseteq F$ is indeed a valid fork on the string $x y$. Moreover, $\hat{F}$ is $x$-balanced since $\tilde{F}_{y}$ is balanced. The claim in Theorem $\square$ follows immediately since $|y| \geq k$. 